
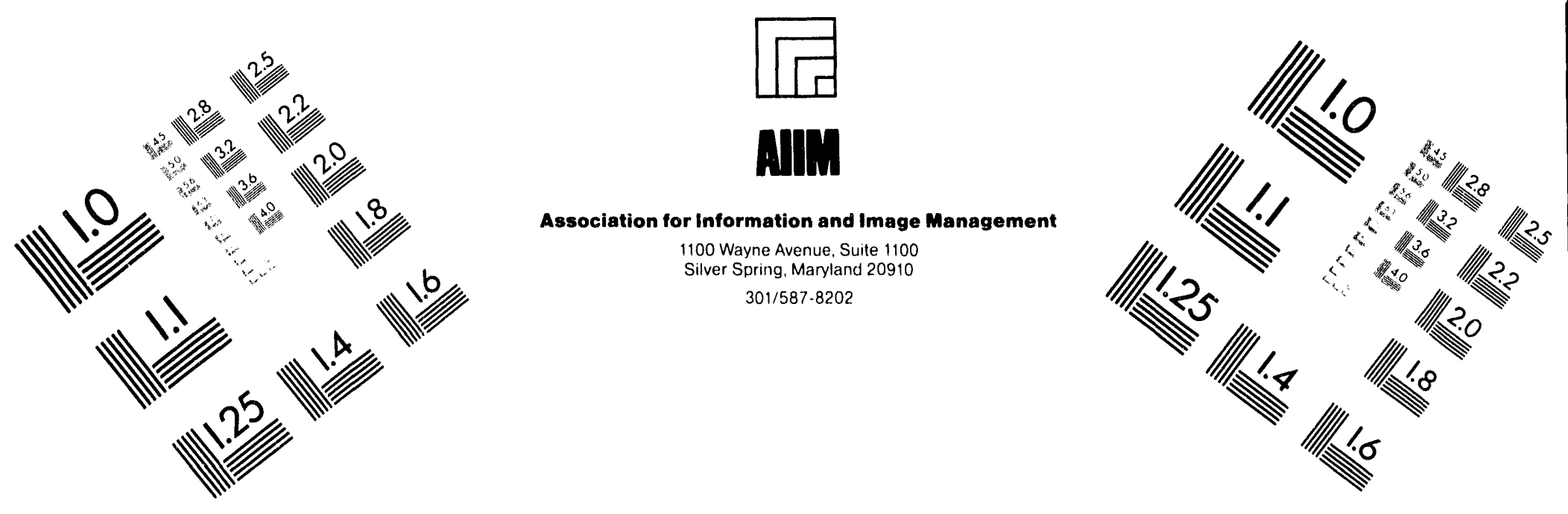

\title{
Centimeter
}

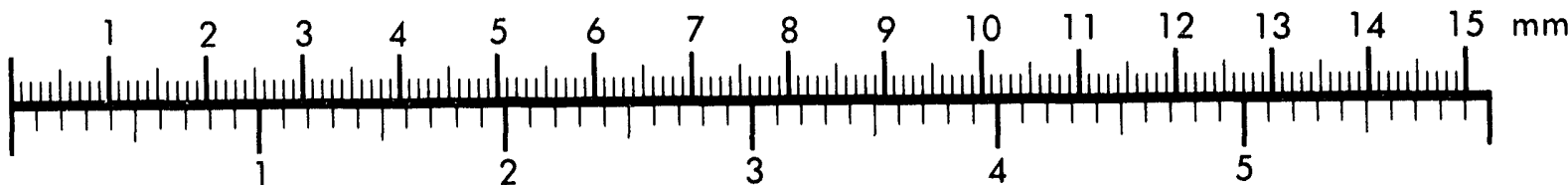

Inches
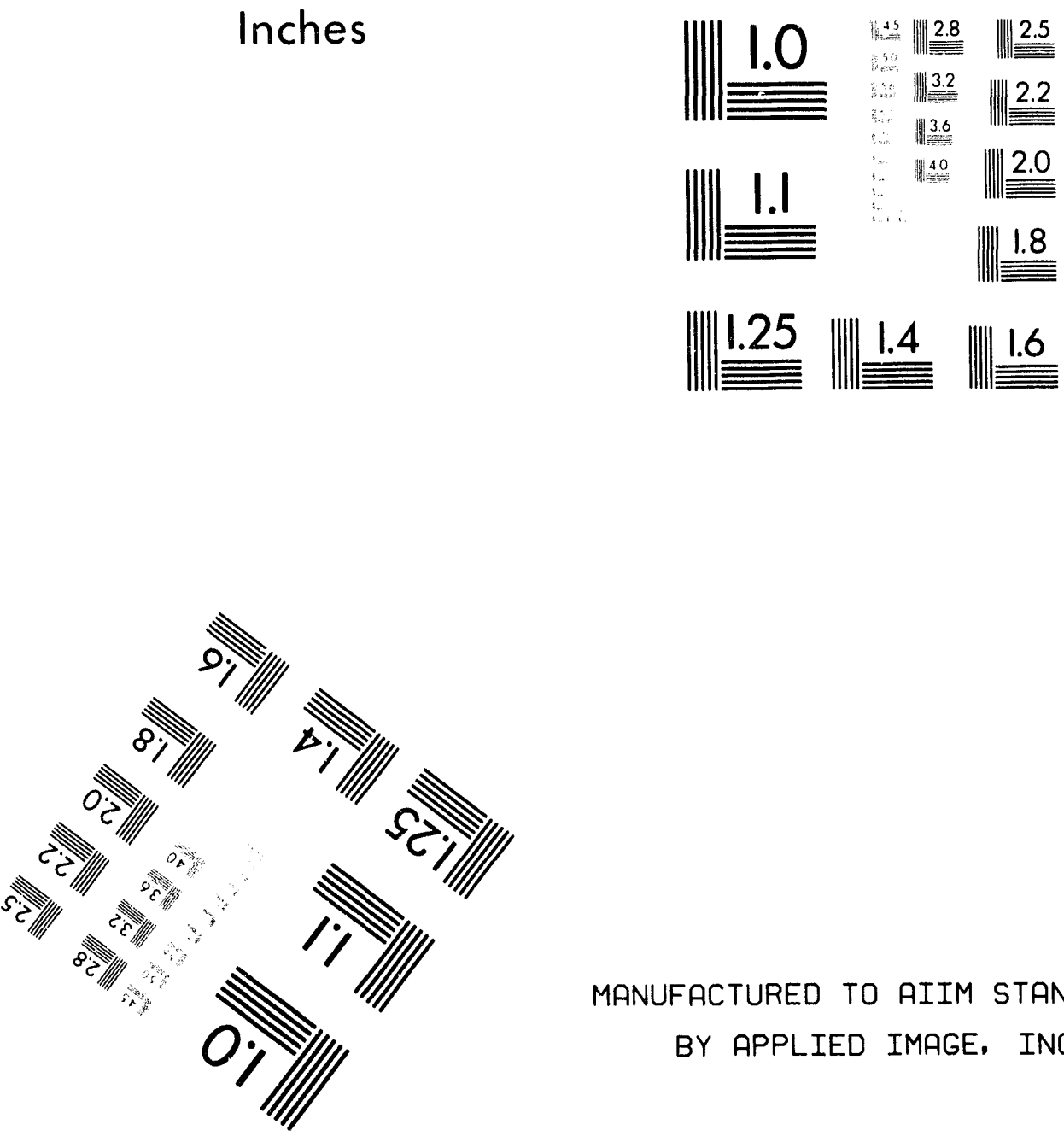

MANUFACTURED TO AIIM STANDARDS

BY APPLIED IMAGE. INC.

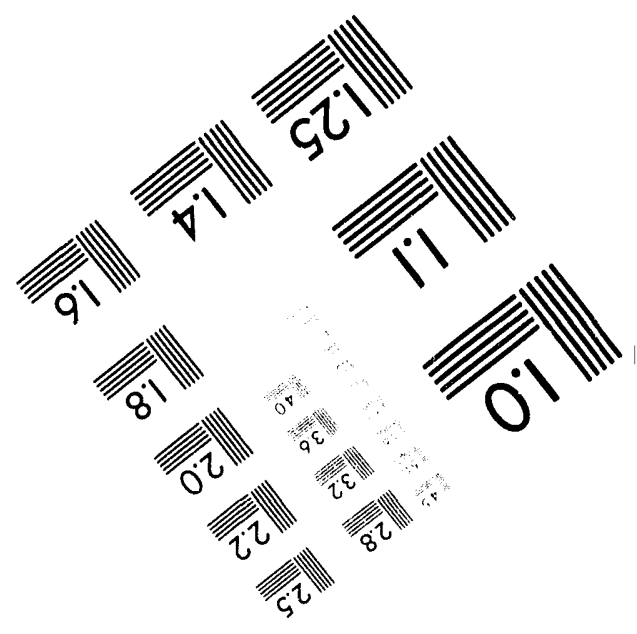



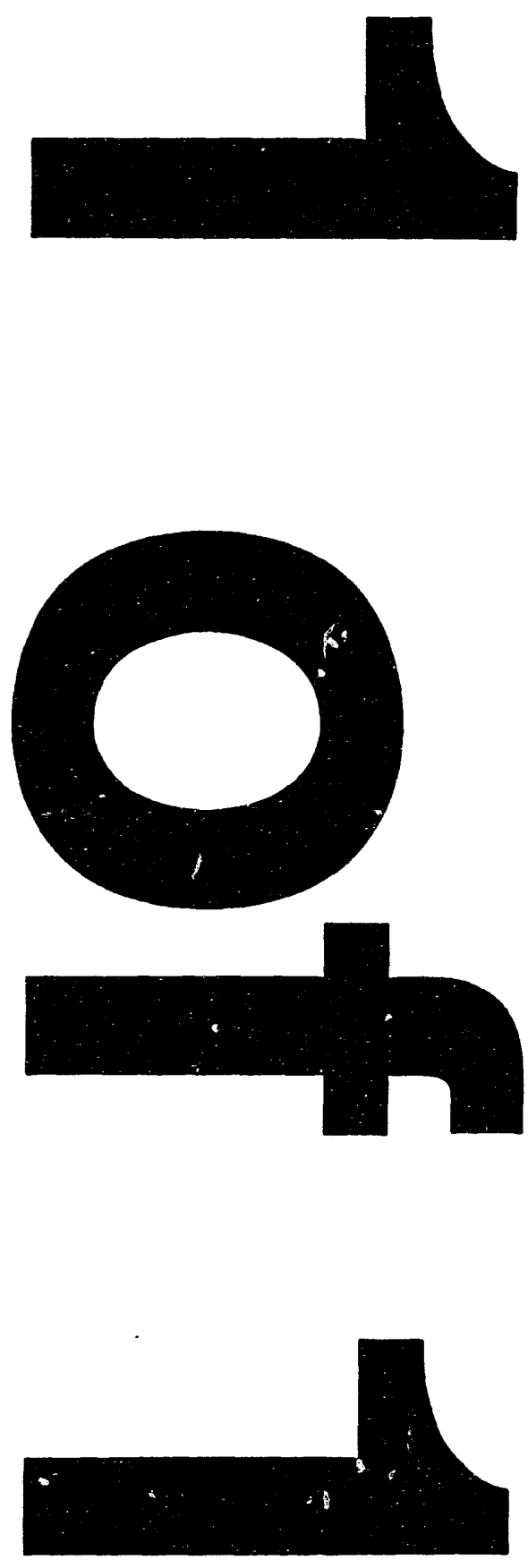
UC-706

Issued: May 1994

\section{Interchangeable Spline}

Reference Guide

Ronald M. Dolin

The WX-Division Integrated Software Tools Team

Jose Arellano

Ralph Gladfelter

John Hopkins

Robert Montoya

Kent Musgrave

Scott Parkinson

Bob Reinhart

Mike Smith

Royce Tyler 


\section{TABLE OF CONTENTS}

PAGE

FRONT COVER

Inside Front Cover....................................................................... ii

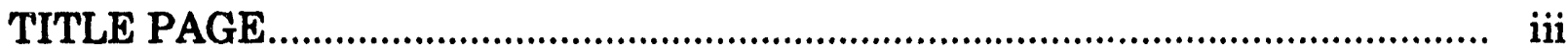

Inside Title Page....................................................................... iv

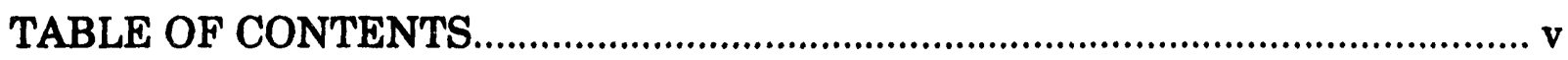

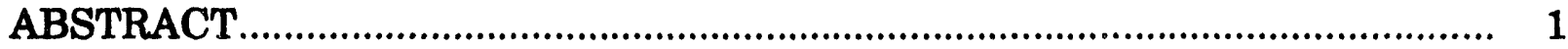

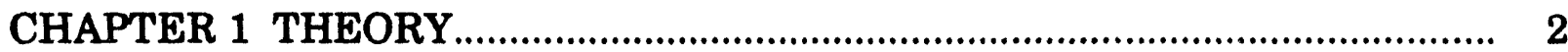

A. Definitions And Problem Statement.................................... 2

B. Proposed Solution................................................................. 5

C. Spline Equivalence............................................................... 5

D. Spline Equivalence Module ................................................ 9

E. CAE system Module....................................................... $\quad 10$

F. Spline Equivalence Module Requirements.......................... 11

G. Software Systems Used........................................................ 11

H. Verification and Validation............................................... 11

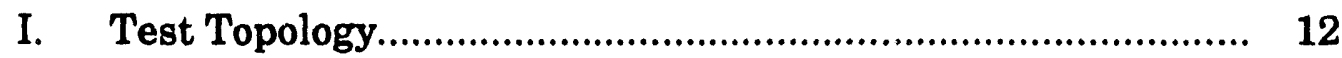

J. Numerical Test Results...................................................... 12

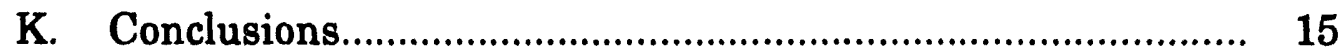

CHAPTER 2 SOFTWARE PROJECT PLAN .................................................... 16

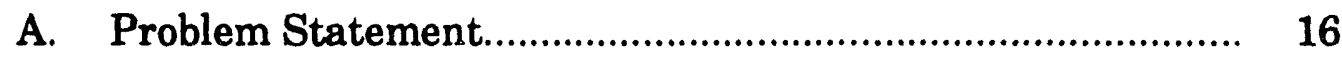

B. Project Goal................................................................... 16

C. Special Considerations...................................................... 16

D. Proposed Plan.................................................................... 16

E. Schedule...................................................................... 17

CHAPTER 3 SOFTWARE REQUIREMENTS SPECIFICATION...................... 19

A. History of Spline Usage................................................... 19

1. Problem Statement................................................... 19

2. Proposed Solution........................................................ 21

a. Interchangeable Spline Process.............................. 21

b. Spline Equivalence Module.................................... 21

c. Spline Data Base Module......................................... 21

d. CAE System Module................................................ 22

e. Spline Usage Document......................................... 22

f. Test/Verification................................................... 22

g. Quality Assurance.................................................. 22

B. Interchangeable Spline Process........................................... 23 
1. Function Definitions..................................................... 23

a. Spline Equivalence Module Functional Definition... 23

b. SE module When Processing a WF spline............... 24

c. SE module When Processing a CAE spline............. 24

d. CAE system Module Functional Definition............. 24

e. WFSYS Module Functional Definition.................... 24

f. Process Control Functional Definition..................... 25

g. Process Controller Executed From Within SE module............................................... 25

h. Process Controller Executed Within WFSYS........... 25

i. Process Controller Executed When CAE system Requests a WFSPL Spline.................. 26

j. Process Controller Executed When CAE System Requests a CAE spline Be Processed......... 26

2. General Design........................................................ 27

a. SE module When Processing a WF spline............... 27

b. SE module When Processing a CAE spline............ 27

C. Spline Equivalence Module Requirements............................. 29

1. Languages And Platforms........................................... 29

2. Documentation, QA And Training................................ 31

3. Baseline Product Definition........................................... 31

4. Multiple Spline Representations................................... 31

5. Data Exchange............................................................. 32

6. Geometry Constraints................................................... 32

7. Software Access............................................................. 32

8. Convergence Measures................................................. 32

9. Interchangeable Spline Program.................................. 32

D. Spline Data Base Module Requirements............................... 32

E. CAE Interface Requirements................................................ 33

1. Languages And Platforms........................................... 33

2. Mode Of Operation.......................................................... 33

3. Data Exchange............................................................. 33

F. Test/Verification Plan Requirements................................... 33

G. Quality Assurance Requirements....................................... 33

1. Software Project Plan (SPP)........................................... 34

2. Software Requirements Specifications (SRS)................ 34

3. Software Design Description (SDD).............................. 34

4. Software Verification and Validation Plan (SVVP)........ 34

5. Software Verification and Validation Report (SVVR).... 34 
CHAPTER 4 SOFTWARE DESIGN DESCRIPTION........................................ 35

A. Main Driver Module........................................................... 36

1. BASELINE_GEN......................................................... 36

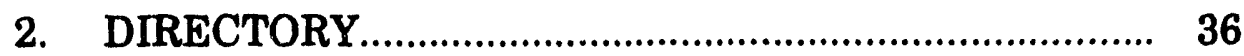

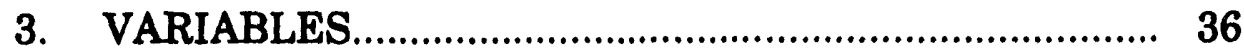

B. IS_Evaluation Module..................................................... 36

1. EVALUATE ........................................................... 37

2. EQUIVALENCE................................................... 38

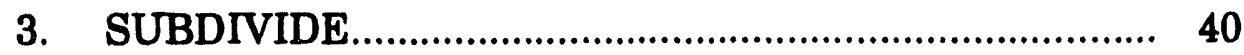

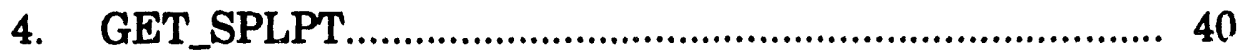

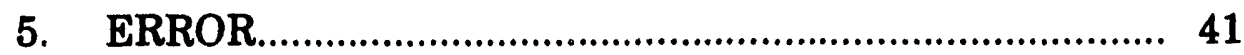

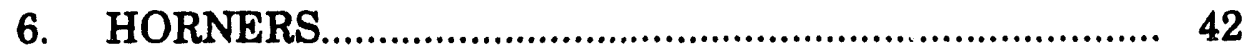

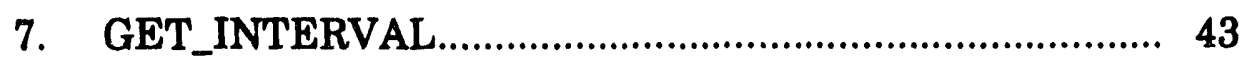

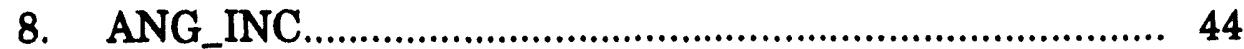

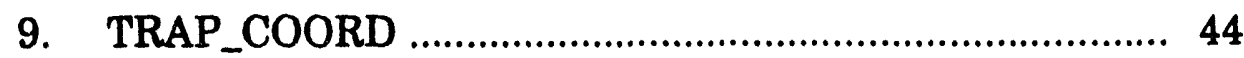

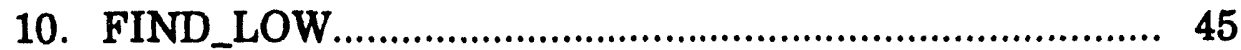

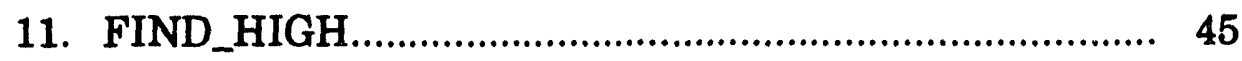

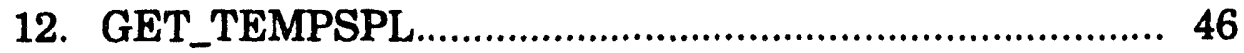

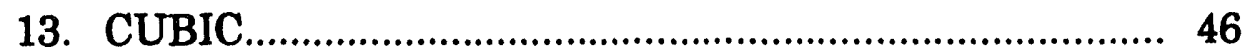

14. SPAWN_PROC..................................................... 47

C. APT Wilson-Fowler Spline Module ..................................... 47

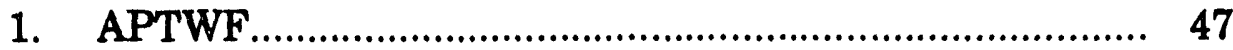

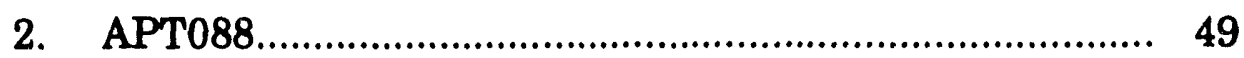

3. APT089

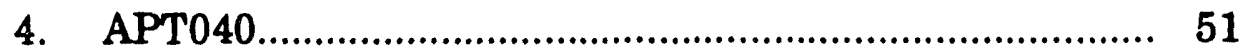

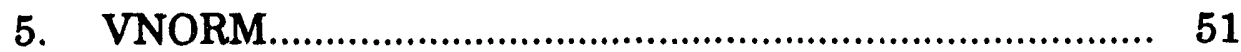

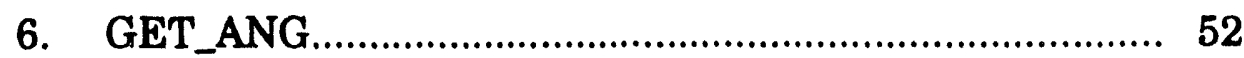

D. INPUT/OUTPUT MODULE......................................... 52

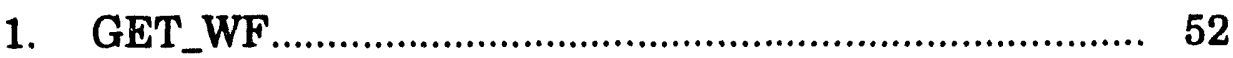

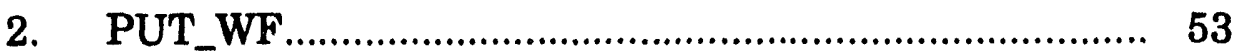

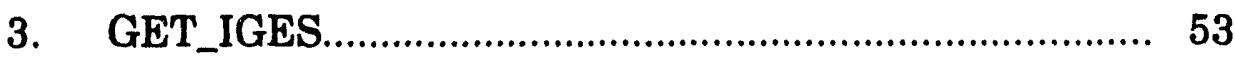

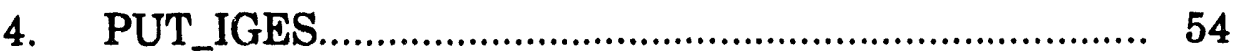

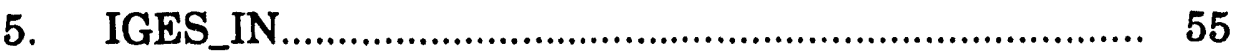

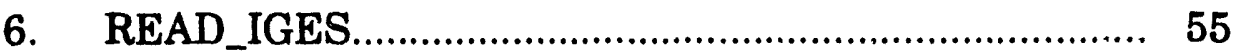

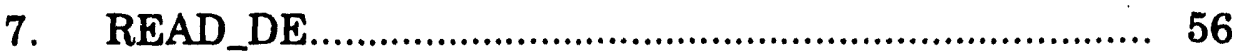

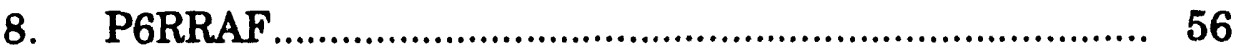

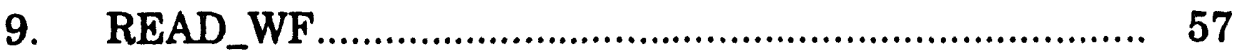

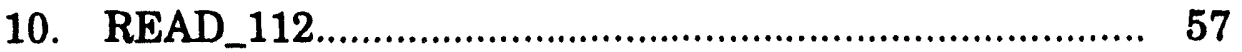

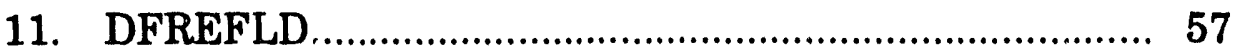

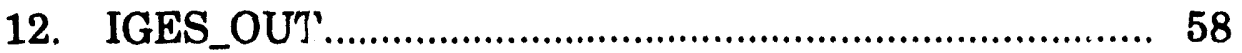




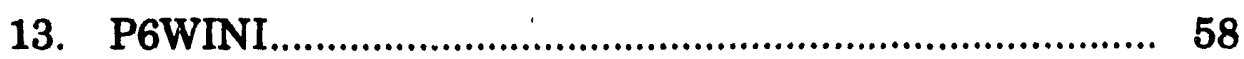

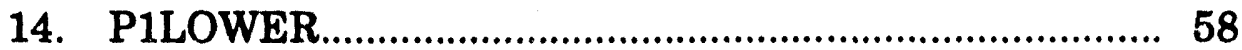

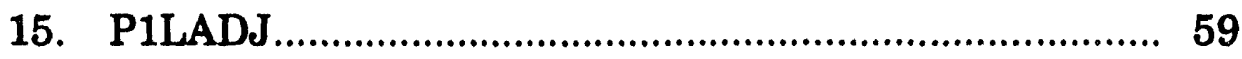

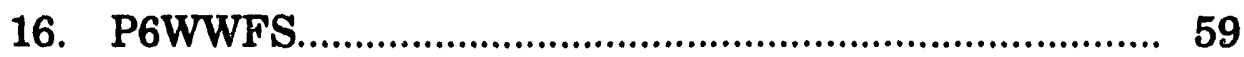

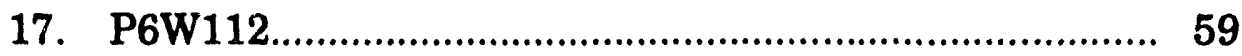

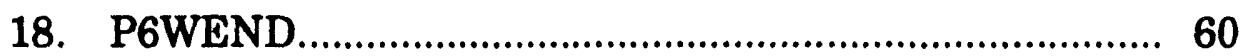

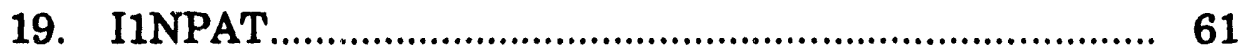

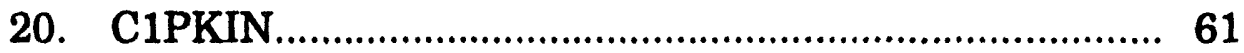

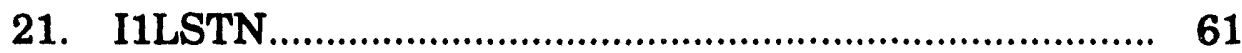

22. F5ATAN............................................................ 61

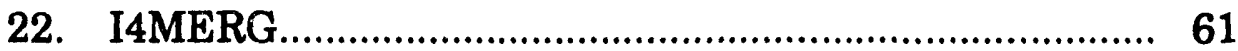

CHAPTER 5 SOFTWARE VERIFICATION AND VALIDATION PLAN............ 62

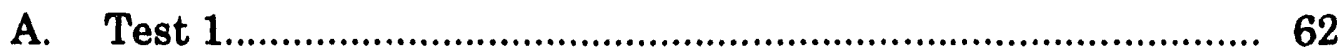

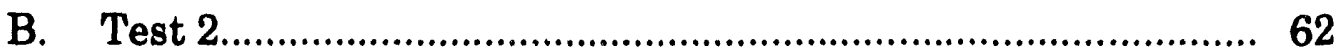

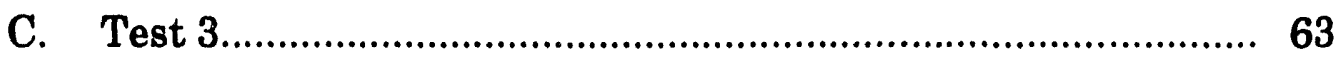

CHAPTER 6 SOFTWARE VERIFICATION AND VALIDATION REPORT........ 64

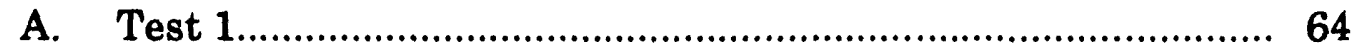

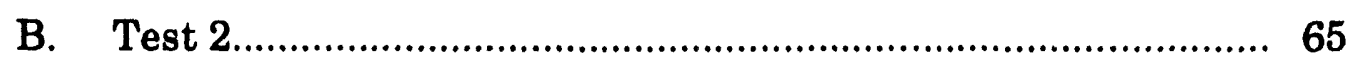

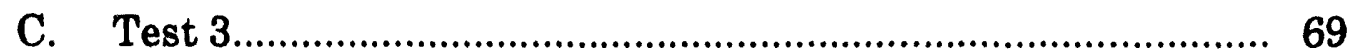

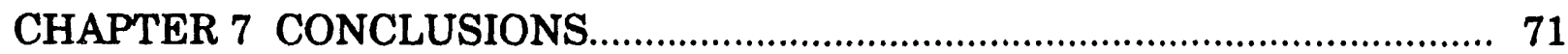


INTERCHANGEABLE SPLINE REFERENCE GUIDE

By

Ronald M. Dolin

and

\title{
The WX-Division Integrated Software Tools Team
}

Jose Arellano, Ralph Gladfelter, John Hopkins,

Robert Montoya, Kent Musgrave, Scott Parkinson,

Bob Reinhart, Mike Smith, Royce Tyler

\begin{abstract}
The WX-Division Integrated Software Tools (WIST) Team evolved from two previous committees. First was the W78 Solid Modeling Pilot Project's Spline Subcommittee, which later evolved into the WX-Division Spline Committee. The mission of the WIST team is to investigate current $\mathrm{CAE}$ engineering processes relating to complex geometry and to develop methods for improving those processes.

Specifically, the WIST team is developing technology that allows the Division to use multiple spline representations. We are also updating the contour system (CONSYS) data base to take full advantage of the Division's expanding electronic engineering process. Both of these efforts involve developing interfaces to commercial CAE systems and writing new software.

The WIST team is comprised of members from WX-11, -12 and 13. This "cross-functional" approach to software development is somewhat new in the Division so an effort is being made to formalize our processes and assure quality at each phase of development. Chapter one represents a theory manual and is one phase of the formal process. The theory manual is followed by a software requirements document, specification document, software verification and validation documents.
\end{abstract}

The purpose of this guide is to present the theory underlying the interchangeable spline technology and application. Verification and validation test results are also presented for proof of principal. 


\section{CHAPTER 1 THEORY}

Splines are used in engineering design for product definition and in manufacturing to drive machining and inspection processes. Splines are most often used to represent free-form topology. Different spline algorithms can represent the same set of defining point data with different free-form curves.

\section{A. Definitions And Problem Statement}

Splines are used to approximate a set of defining point data with a continuous curve. Splines are most often used to represent free-form topology.

If a spline algorithm uses a set of defining point data to generate a free-form curve that passes through each of the defining points, the defining point data are referred to as through points. In other words, a spline generated using a set of through point data passes through each of the defining points.

If a spline algorithm uses a set of defining point data to generate a free-form curve that does not necessarily pass through the defining points, the defining point data are referred to as control points. In other words, a spline generated using a set of control point data does not necessarily pass through those points.

Figure 1.1 shows two different spline topologies generated using the same set of defining point data. The spline algorithm that generated the solid curve topology passes through each defining point while the spline algorithm that produced the dashed curve topology does not.
Depending on the spline algorithm used and how its available parameters are specified, there are potentially many different curve topologies that can be approximated from a single set of defining point data.

Some spline algorithms generate topologies that pass through the defining points while others do not.

Some spline algorithms may approximate a given defining point interval with a convex curve topology. Alternatively, other spline algorithms may approximate the same defining point interval with a concave curve topology or a topology containing both a concave and convex topology (i.e., an inflection point).

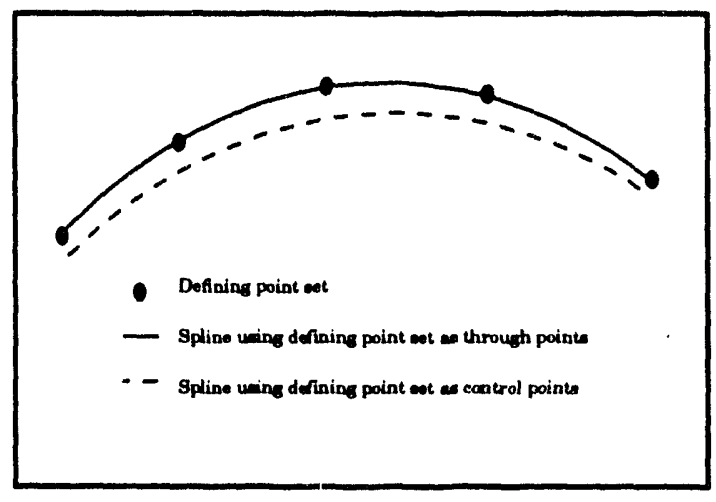

Figure 1.1. Example of how two different type spline algorithms can use the same set of defining points to generate free-form curve topology. 


\section{All spline algorithms share the same underlying mathematical concept - minimizing energy.}

Spline algorithms take a given set of defining point data and attempt to construct a minimum energy curve. The topology between defining point pairs can vary with different spline algorithms depending upon how a given spline algorithm attempts to minimize energy.

Most spline algorithms use higher ordered polynomials to generate approximate topology between defining point pairs. For example, the family of spline algorithnus known as cubic splines use third order polynomials to define the shape of the spline topology.

Polynomial representations allow robust spline topology with a relatively small set of defining points.

Figure 1.2 shows three different polynomials and the potential topological shapes each polynomial can generate. A linear polynomial can generate a line segment connecting two points. A quadratic polynomial can generate either a concave or convex shape topology between two points. The cubic polynomial can generate a concave and convex shaped topology containing one inflection point.

The polynomial representation used by most spline algorithms allows a set of defining points to be fit with a certain degree of continuity. Cubic splines are second order continuous. This means that a cubic spline approximation is continuous in position, slope (first derivative), and curvature (second derivative).

Continuity is insured by forcing the slope and curvature at the end of one interval to match the slope and curvature at the beginning of the next interval. Usually this matching procedure is iterative.

Figure 1.3 shows two intervals of a spline. The slope at the end of the Ith interval is labeled $\mathrm{TB}_{\mathrm{I}}$ and the slope at the start of I+1st interval is labeled $\mathrm{TA}_{\mathrm{I}+1}$. Using an iterative process the spline topology can be modified th allow $\mathrm{TB}_{\mathrm{I}}$ and $\mathrm{TA}_{\mathrm{I}+1}$ to match. If the spiine's defining polynomial is a cubic, the iterative process also attempts to concurrently match curvatures.
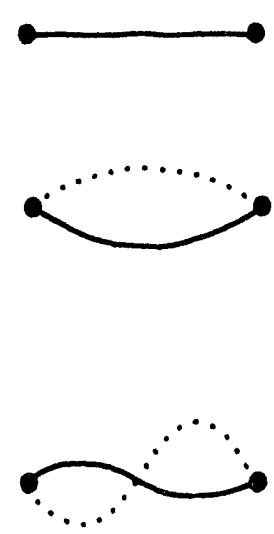

CUBIC

$A s^{3}+B n^{2}+C B+D$

LINEAR:

$\mathrm{CB}+\mathrm{D}$

QUADRATIC: $\mathrm{Bs}^{2}+\mathrm{Cs}+\mathrm{D}$

Figure 1.2. Three different polynomial expressions and the topologies they can produce.

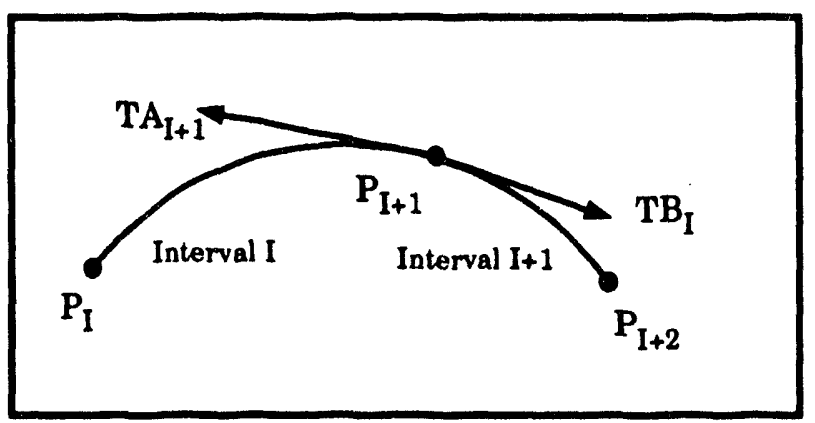

Figure 1.3. Matching tangents between the Ith and $I+1$ st spline intervals. 
There are a host of different spline algorithms available. Most spline algorithms are superior to other spline algorithms in at least some aspect of spline representation and manipulation. Within WX-Division, the Wilson-Fowler (WF) spline and the B-spline family of splines are of greatest interest.

The WF spline represents the Division's current spline implementation as well as the DOE/NWC's current spline standard.

B-splines are becoming an industry standard. They represent the direction that the Division should be aiming its long-term spline objectives. The B-spline family of spline algorithms includes rational B-splines, non-rational B-splines and non-uniform rational B-splines (NURBS).

WX-Division is evaluating current engineering processes. Where necessary, old processes are evolving to accommodate newer computer enhanced engineering methodologies.
New methodologies should not be forced to support only WF splines. The WF spline algorithm is only twodimensional. This means that WF splines cannot be utilized with commercially available solid modeling technology.

Anticipating that a B-spline based CAE system will become the Division's standard, potential problems concerning product definition, data transfer, manufacturing, and inspection need to be addressed.

The foremost problem deals with the different spline algorithms that use the same set of defining point data to produce topologies that are each taken to represent product definition.

Another problem deals with using non-WF CAE systems while attempting to maintain an historical product definition data base.

\section{Cubic Spline Are Continuous In Position, Slope And Curvature}

The cubic polynomial equations for a two-dimensional spline are:

$$
\begin{array}{ll}
x=A_{x} S^{3}+B_{x} S^{2}+C_{x} S+D_{x} & y=A_{y} S^{3}+B_{y} S^{2}+C_{y} S+D_{y} \\
x^{\prime}=3 A_{x} S^{2}+2 B_{x} S+C_{x} & y^{\prime}=3 A_{y} S^{2}+2 B_{y} S+C_{y} \\
x^{\prime \prime}=6 A_{x} S+2 B_{x} & y^{\prime \prime}=6 A_{y} S+2 B_{y} \\
& \text { where } 0 \leq S \leq L, \\
& \text { and } L=\text { length of the interval }\left(x_{n}-x_{n-1}\right)
\end{array}
$$

- The first set of equations, for $x$ and $y$, represent the position along the spline curve in the interval between $X_{n-1}$ and $X_{n}$ for any bounded value of $S$.

- The second set of equations, for $x^{\prime}$ and $y^{\prime}$, represent the slope of the spline curve at any valid position in the defining interval.

- The third set of equations, for $x^{\prime \prime}$ and $y^{\prime \prime}$, represent the curvature of the spline curve at any valid position in the defining interval. 


\section{B. Proposed Solution}

One solution to the problems described earlier is to develop a procedure that would allow different spline algorithms to produce equivalent topologies when used with a given set of defining point data. This solution is referred to as Spline Equivalence.

Spline Equivalence insures that two different spline algorithm approximations to a single set of defining point data render equivalent topologies at the defining points.

Spline Equivalence can also insure that one spline algorithm approximation to a set of defining point data renders a topology that is equivalent to the topology rendered by a different spline algorithm approximation to a different set of defining point data.

In the Spline Equivalence process, when two different spline algorithm approximations are generated, the topologies are equivalent at the defining points and everywhere in between the defining points.

\section{Spline Equivalence}

The premise of spline equivalence is that a curve topology generated from a sufficiently well represented set of defining points can be independent of the spline algorithm used to generate it. Spline Equivalence requires that the number of points used to define a spline be allowed to vary.

The goal of the spline equivalence technology is to generate a set of defining point data such that when two different spline algorithms build approximate topology from the defining point set they produce curve topologies that are equivalent.

If a set of defining point data has an equivalent representation, the defining point data is invariant under spline transfer. In other words, different spline algorithms fit the defining point set with equivalent topology. Different versions of the same spline algorithm fit the defining point set with an equivalent topology. The same spline algorithm on different platforms fits the defining point set with an equivalent topology.

Two different spline topologies are said to be equivalent when their maximum linear deviation is less than an equivalence error. The equivalence error for WX-Division is presently defined as $0.00025 \mathrm{~mm}$. This value is one order of magnitude greater than the precision of a production inspection machine and two orders of magnitude greater than the precision of a production machining tool.

Figure 1.4 shows two different spline algorithm approximations to a given interval of a defining point set and how the linear deviation is measured.

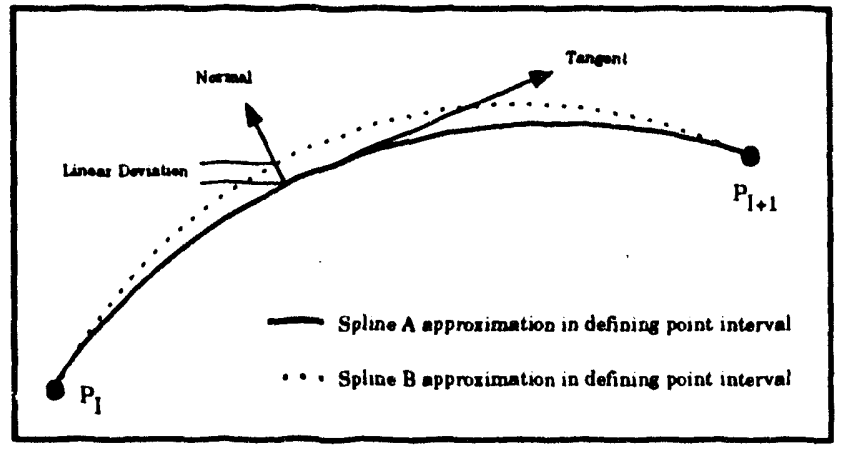

Figure 1.4. Measuring the linear deviation between two different spline approximations in a given spline defining point interval $\left(P_{I}, P_{I+1}\right)$. 
Since the equations describing the spline topology are known in any interval, any position within an interval can be measured. Linear deviation is measured by computing the normal at a given location on one spline curve and projecting it through the other spline.

The distance between the location on the first curve where the normal was taken and the location on the second curve where the normal intersected it is referred to as the linear deviation. The linear deviation is computed using the standard distance equation:

$$
d=\left[\left(X_{2}-X_{1}\right)^{2}+\left(Y_{2}-Y_{1}\right)^{2}+\left(Z_{2}-Z_{1}\right)^{2}\right]^{1 / 2}
$$

Given a set of defining point data, spline algorithms attempt to construct minimum energy curve wopologies. The topology generated between defining point pairs can vary with different spline algorithms. As more points are added to a defining point set the distance between points decreases and the amount of variation between topologies generated by the different spline algorithms decreases.

The spline equivalence hypothesis states that as the distance between defining points decreases, the variation between topologies generated by different spline algorithms using the set of defining point data also decreases.

Most spline algorithms use higher order polynomials to approximate topology between defining point pairs. For example, cubic splines use third order polynomials.

Polynomial representations allow robust topology with a relatively small defining point set. As more points are added to an existing defining point set the order of the spline could be reduced because the energy available within each interval is decreased. A zero energy curve is a straight line.

In the limit, enough defining points could be specified that a linear point-to-point approximation would yield a reasonable topological representation.

An analogy to this hypothesis can be made using common graphical representation schemes. A method for displaying circles in a graphical representation system is to compute the coordinates for a set of points that lie on the circle, and then connect those points with straight line segments.

The more points used to represent the circle the more the straight line segments appear to capture the circle's shape. While every point along a circle is known, there is a practical limit on how many straight line segments should be used to approximate it. That limit is usually dependent upon the necessary resolution.

In the case of spline representation the practical limit would be determined by two primary factors. The first primary factor is the number of intervals necessary to bring two spline representations into equivalence. The second primary factor is the proximity of defining points.

Implementation of the spline equivalence theory begins with an initial set of defining point data and the approximate spline topologies generated by the two different spline algorithms fitting that set. One of the spline 
algorithm approximations is referred to as the baseline representation.

The goal of Spline Equivalence is to build a new defining point set such that when used by two different spline algorithms, their generated topologies are equivalent. This approach suggests that both spline algorithms would use the new set of defining point data.

A second approach to achieving spline equivalence is to consider the initial set of defining point data and the baseline spline's approximation using those points as one topology and a second set of defining point data with a second spline's approximation using those points as another topology.

In this second approach, the goal of spline equivalence is to generate a second set of defining point data such that when used by the second spline algorithm, the resulting topology is equivalent to the baseline spline's topology using the initial defining point data.

This approach suggests that there are two sets of defining point data. The first set is the initial defining point data used to generate the baseline topology and the second set is the defining point data developed to construct a topology that would be equivalent to the baseline.

There are advantages and disadvantages to both approaches. An advantage of the first approach is that a single set of defining point data can be used to generate approximate topology using two different spline algorithms. Both topologies will be equivalent to the baseline topology. This approach allows for a bi-directional mode of product definition.

A disadvantage to the first approach is that the final set of defining point data is different than the initial set. Another disadvantage is that the final topologies of both spline algorithm approximations are only equivalent to the initial topology of the baseline spline.

A third disadvantage is that equivalence had to be created for two spline topologies, which could drive up the number of points in the final defining point set.

An advantage of the second approach is that the baseline spline algorithm's approximation uses the initial set of defining point data.

A second advantage is that only the second spline topology is considered when constructing the second set of defining point data. This should result in a set of defining points that is smaller than the set generated using the first method.

A disadvantage of the second approach is that there are two defining point data sets that have to be managed. This forces a uni-directional mode of product definition, as opposed to the bi-directional mode of product definition resulting from the first approach.

It is not clear in this second approach how one would modify the baseline spline definition to represent a modification made to the second spline definition's topology.

In a heterogeneous environment, the bi-directional mode of product definition is necessary. 
Figures 1.5 through 1.9 demonstrate the second approach (because it provides a simpler pictorial description). Figure 1.5 shows two different spline algorithm approximations to an initial defining point set over the Ith interval. The linear deviation between the two spline topologies exceeds the equivalence error somewhere within the interval.

In an effort to bring the second spline approximation's topology within equivalence of the baseline topology, a new point is added to the second spline's defining point data set. That new point is added along the baseline spline's approximating curve near the center of the interval in the second spline that is being subdivided.

Figure 1.6 shows the same baseline spline's Ith interval and the second spline's two new intervals (ith and $i+1$ st). As shown, the second spline approximation's ith interval exceeds the equivalence error.

A new point is added to the second spline's defining point data set. The new point's coordinates are computed by projecting a normal from the center of the ith interval of the second spline through the baseline curve. The intersection point becomes the new defining point for the second spline.

Three issues should be discussed. First, every time a new point is added to the second spline's set of defining point data, the coordinates are computed using the baseline spline topology.

The second issue is that only one new point is added to the second spline definition per evaluation. This is because splines are pathological. There is no way to anticipate what impact a new point will have on a spline approximation.

The third issue is the location of the new point. Since splines are pathological and there is no way to anticipate the impact of adding a new point there is no need to expend resources attempting to find the optimal location for a new point. Instead, intervals that are not equivalent are subdivided in the middle.

Figure 1.7 shows the baseline spline approximation over its Ith interval and the three intervals of the second spline over the same span.

The ith interval of the second spline is now equivalent to the baseline spline. However, the $i+1$ st interval is out of tolerance so must be subdivided.

Every time a new point is added to the second spline approximation's defining point data set the entire spline is re-evaluated: even intervals that previously converged. This is because of the pathological nature of splines and the unknown effects that adding new points has on the overall topology.

Figure 1.8 shows the baseline spline approximation over its Ith interval and the four intervals of the second spline approximation over the same span.

Now, the $i+3$ rd interval is not equivalent and a new point is added by projecting a normal from the center of the $i+3 r d$ interval through the baseline spline topology. The coordinates of the new point are the coordinates of the intersection between the baseline spline and normal. 
Figure 1.9 shows the effects of adding the new point over the second spline's approximation. The second spline has now converged and is equivalent to the baseline spline approximation. The baseline spline used two defining points to approximate the span shown.

The second spline required five defining points to approximate the same span with a topology equivalent to the baseline.

The spline equivalence theory can be validated using numerical tests with analytical topology. Results of the validation tests are presented in section

The impetus for the spline equivalence capability is that once implemented each NWC agency is free to use whatever spline algorithm they prefer provided each spline is processed using the SE module.

\section{Spline Equivalence Module}

There are three spline algorithms used by the spline equivalence module, the B-spline, the Automatically Programmed Tools (APT) spline and the general Wilson-Fowler spline.

A B-spline is generally (n-1)-order continuous. A cubic B-spline $(n=3)$, is second order continuous. This means that the spline is continuous in position, slope and curvature.

The Automatically Programmed Tools (APT) software is used in manufacturing and inspection processes by the NWC. APT has an integrated WF spline algorithm. The version of APT used by the Spline Equivalence module is the CAMI-APT.

The Spline Equivalence (SE) module contains an integrated WF

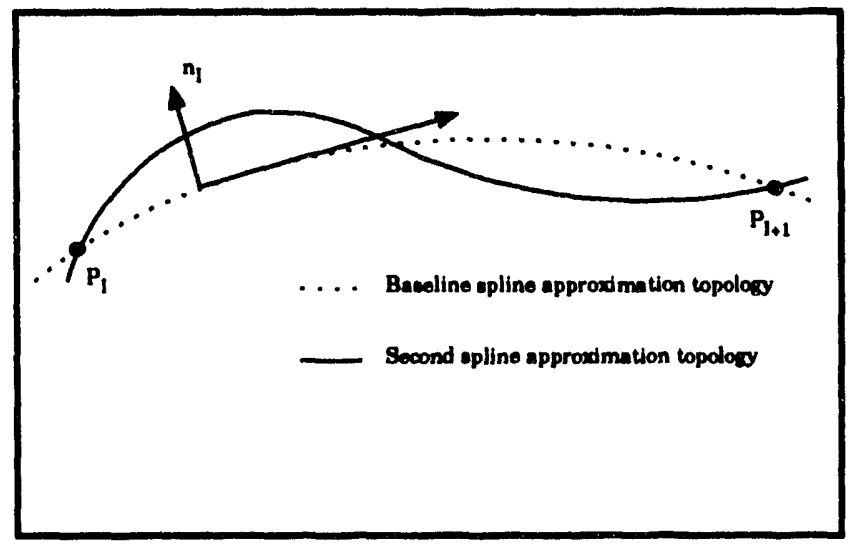

Figure 1.5. Computing linear deviation between baseline and second spline approximation topologies in Ith spline interval.

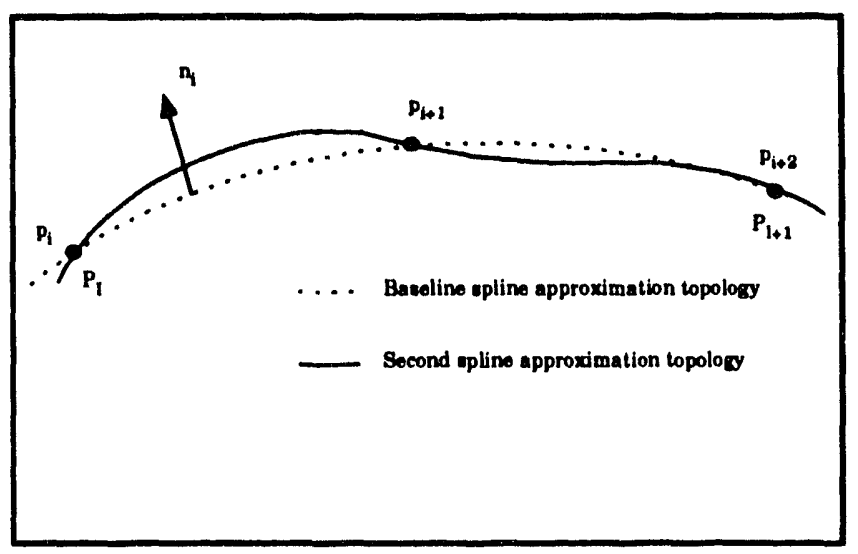

Figure 1.6. Computing linear deviation between baseline and new ith interval of second spline.

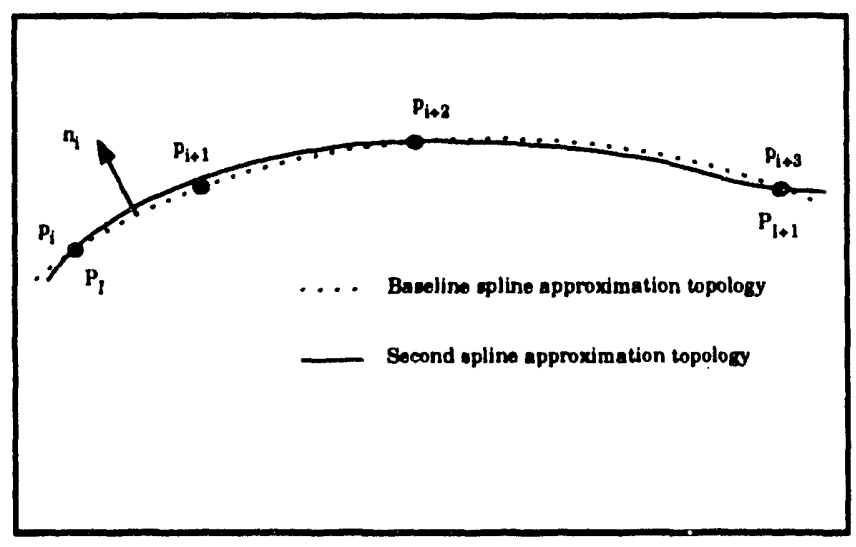

Figure 1.7. Computing linear deviation between baseline and new ith interval of second spline. 
spline algorithm that is used to generate baseline WF spline representations.

The SE module is a stand alone program. In other words, it is not dependent upon any CAE system module. This gives the SE module an ability to be shared by DOE/NWC agencies without having to share the same CAE system.

The SE module stands alone because module interactions are handled by a process controller and data exchange between modules is done with file transfers. The SE module reads and writes files, so to use it one only needs to understand what the $\mathrm{I} / \mathrm{O}$ files look like and how to access them.

The SE module's only capability is to process splines and make them interchangeable between a baseline spline and another spline algorithm.

In one case the internal WF spline is the baseline and a CAE system spline is made equivalent to it. In another case, the CAE spline is taken as the baseline and the internal WF spline is made equivalent.

The SE module ensures that a spline is within a specified tolerance of the baseline representation.

\section{E. CAE System Module}

The CAE system will input spline data in an ASCII XY-data format. The CAE system's internal spline algorithm will generate a spline using the information obtained from the input file. The CAE system will output a full IGES representation of its computed spline (defining-points, coefficients and parameterization).

The IS process is not dependent upon any particular CAE system, only the required CAE system functionality.

A Process Contoller (PC) automates the IS process. The PC is used to interact between the CAE system module and the SE module.

For more information, see the Interchangeable Spline's Software Requirements Document.

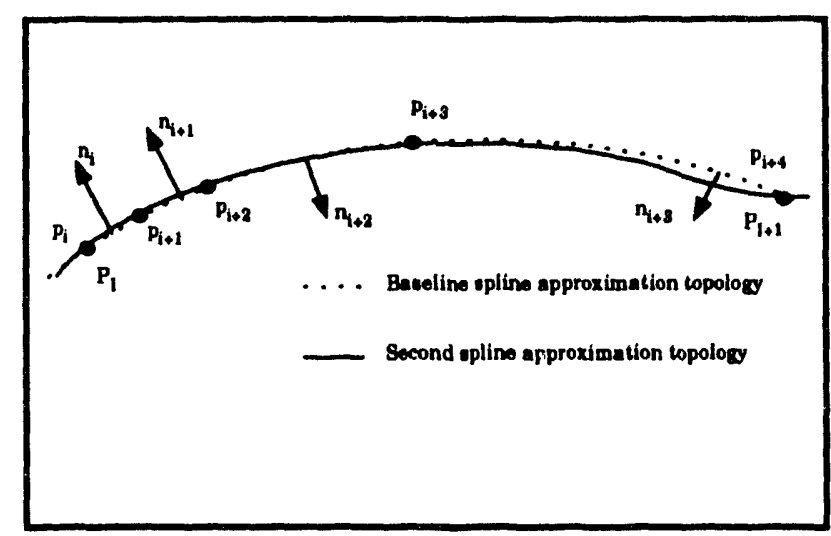

Figure 1.8. Computing linear deviation between baseline and new ith to $i+3$ rd interval of second spline.

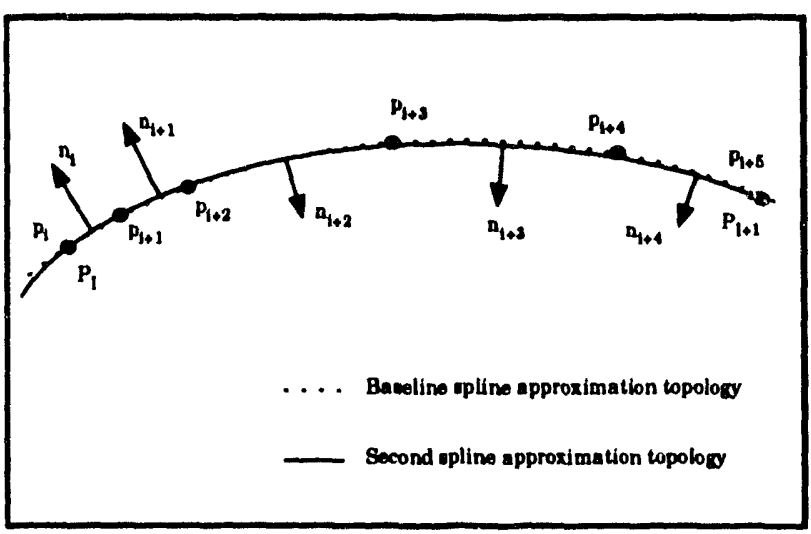

Figure 1.9. Computing linear deviation between baseline and new ith to $j+4$ th interval of second spline. 


\section{F. Spline Equivalence Module Requirements}

The interchangeable spline (IS) process contains a Spline Equivalence (SE) module and a CAE System module. The Spline Equivalence module has an integrated Wilson-Fowler spline algorithm.

A post processing program was written to determine how well a spline algorithm approximates an analytical curve. Some measures of a spline approximation's accuracy are its linear deviation from the analytical curve (Error), mean of the error and standard deviation of the error.

The maximum deviation from the analytical curve (error), is a measure of how far the spline approximation is from its analytical curve. Each spline approximation is sampled at 1000 evenly spaced points.

The minimum distance between the spline approximation and the analytical curve is computed at each sample point. The Error is computed as the maximum of the 1000 minimum errors.

The mean of the error provides an indication of how the spline approximation is converging toward the analytical curve. The standard deviation of the error indicates how the error is distributed.

\section{G. Software Systems Used}

The WX-Division IGES editing and mass property program, IGVIEW, participated in the spline tests. IGVIEW contains an implementation of the Wilson-Fowler spline algorithm written by R. Dolin. ${ }^{1}$

IGVIEW is a single precision program. This means that evaluation results can only be compared to typically six significant digits of accuracy.

The verification and validation tests used the Wilson-Fowler algorithm with default end conditions.

Three commercial computer-aided engineering programs were also used for the verification and validation tests. They were the ICEM, ANVIL and Applicon CAE software systems.

The Applicon CAE system is also known as BRAVO. It contains Uniform B-spline and NonUniform B-spline algorithms. The verification and validation tests used the NonUniform B-spline algorithm.

The ICEM CAE software system has a Wilson-Fowler spline algorithm. The verification and validation tests used all default conditions with circular end angles.

\section{H. Verification And Validation}

The Interchangeable Spline (IS) is tested using four different computer assisted engineering systems and three analytical test topologies to verify the IS hypothesis and validate its software implementation. The software is then further validated by performing numerical tests.

The ANVIL CAE software system has a Wilson-Fowler spline and an piecewise cubic spline algorithm. The verification and validation tests used

1 Dolin, R. M., "The Wilson-Fowler Spline In A Global IGES Coordinate Frame," Los Alamos National Laboratory report number LA-11024MS, September, 1987, Los Alamos, NM, 87545. 
the Wilson-Fowler algorithm with default end conditions.

\section{Test Topology}

Three analytical curves were used for the verification and validation tests. The analytical curves were are a $2 x$ ellipse, 20x ellipse and a sine wave.

The sine wave extends from $0<q$ $<2 \pi$ radians and the other three curves extend From $0<q<\pi / 4$ radians.

Several defining point sets were used to test each analytical curve. The defining point sets started out coarse (ten points) and increased in density.

The numbers of defining points used to approximate the analytical curves are $10,20,30,45,90,180$ and 360 . In all cases, the defining points were evenly distributed.

The tests show that all four spline algorithms reuder spline topologies with errers below the specified threshold. They all display different characteristics, like converging at different rates.

The best spline approximation to the circle appears to occur when the angular increment is approximately two degrees. Decreasing the angular increment past the two degree setting does not seem to improve the spline approximation. This may suggest that there is a point of "diminishing returns" with respect to spline convergence.

For the circle, the two degree angular increment is the same two degree interval typically referred to in contour specification. However, a circle is the only geometry for which this two degree phenomena was observed.

In general, the maximum error occurred at the start of the spline.
Errors at spline end points are common in spline algorithms.

\section{J. Numerical Test Results}

Several numerical tests were designed to further demonstrate the interchangeable spline hypothesis. The first test suite numerically evaluated 1517 design topologies from a data base of engineering designs.

The 1517 splines were tested by first generating the Spline Equivalence's baseline Wilson-Fowler spline and then building an equivalent topology using a CAE system spline. For this experiment, the Wilson-Fowler spline from the IGVIEW software system was used.

Of the 1517 splines processed, 1097 required additional points for the equivalent representation. In other words, 1097 IGVIEW representations required additional defining point information to generate equivalent topology.

For the 1097 modified splines, the mean of the number of additional points was 8.7 and the standard deviation was 13.2. This means that on average, each modified spline required 8.7 additional defining points.

One concern with using the IS technology is that the number of additional points required to generate equivalent topology would be large. This first experiment demonstrates that the number of required additional defining points is not large.

The largest number of additional points was 99 . However, when the baseline topology was investigated, discontinuities were found. When the discontinuities were removed from the 
baseline definition, the number of additional points decreased.

In fact, when all splines containing discontinuities were removed from the initial data base of 1517 splines, the mean dropped off to 6.5 additional points and the standard deviation was 10.3.

This set of experiments matched a Wilson-Fowler spline to a Wilson-Fowler baseline. It demonstrates how different implementations of the same spline algorithm can render different topologies. The experiments also verify that equivalent splines can be generated.

The same experiment were repeated using two commercial $\mathrm{CAE}$ systems. These experiments considered a smaller subset of the spline data base consisting of 66 spline topologies.

The 66 topologies were selected by simply getting all design topologies used recently. They could therefore be considered somewhat random.

The topologies processed using IGVIEW as the CAE module had a 60 percent modification rate. In other words, 60 percent of the topologies processed required additional points to generate an equivalent APT Wilson-Fowler/ IGVIEW Wilson-Fowler spline.

The mean of the number of additional defining points required was 5.2424 and the standard deviation was 5.6325 .

After processing, the mean of the errors in the equivalent splines versus the original splines was $1.8919 \times 10^{-4} \mathrm{~mm}$ and the standard deviation was $0.4703 \times 10^{-4} \mathrm{~mm}$.
The same topologies processed using BRAVO as the CAE module had a 65 percent modification rate. In other words, 65 percent of the topologies processed required additional points to generate an equivalent APT Wilson-Fowler/ BRAVO B-Spline spline.

The mean of the number of additional defining points required was 5.318 and the standard deviation was 5.4155 .

After processing, the mean of the errors in the equivalent splines versus the original splines was $1.9276 \times 10^{-4} \mathrm{~mm}$ and the standard deviation was $0.4698 \times 10^{-4} \mathrm{~mm}$.

The same topologies processed using ICEM as the CAE module had a 71 percent modification rate. In other words, 71 percent of the topologies processed required additional points to generate an equivalent APT Wilson-Fowler/ ICEM spline.

The mean of the number of additional defining points required was

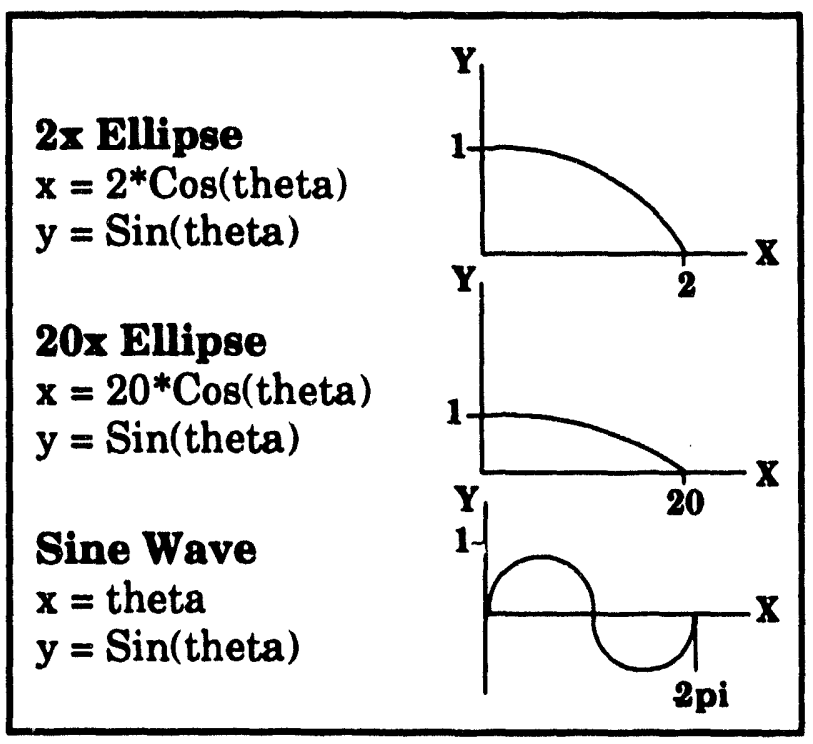

Figure 1.10. Analytical curves used in tests. 
7.0151 and the standard deviation was 6.7766 .

After processing, the mean of the errors in the equivalent splines versus the original splines was $1.9231 \times 10^{-4}$ $\mathrm{mm}$ and the standard deviation was $0.4851 \times 10^{-4} \mathrm{~mm}$.

Table 1.1 lists the results of these numerical tests. The results seem to validate the concept of Spline Equivalence.

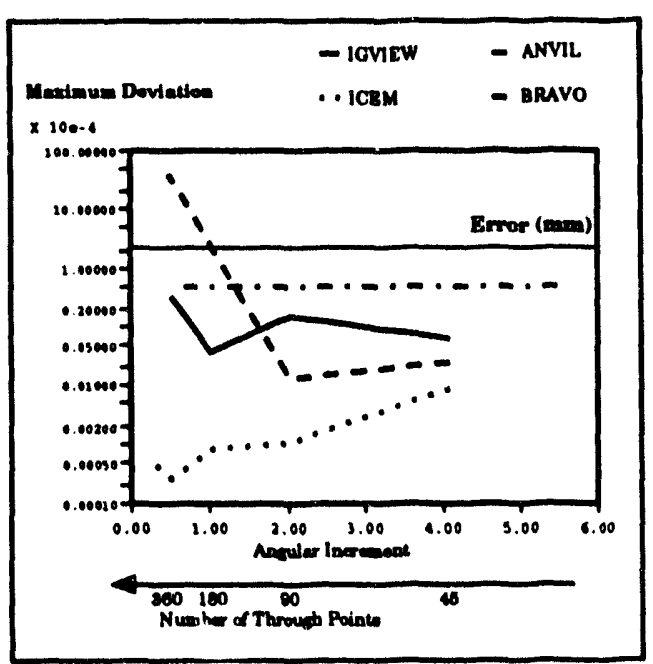

Figure 1.11. Error report for spline approximation to a $2 x$ ellipse.

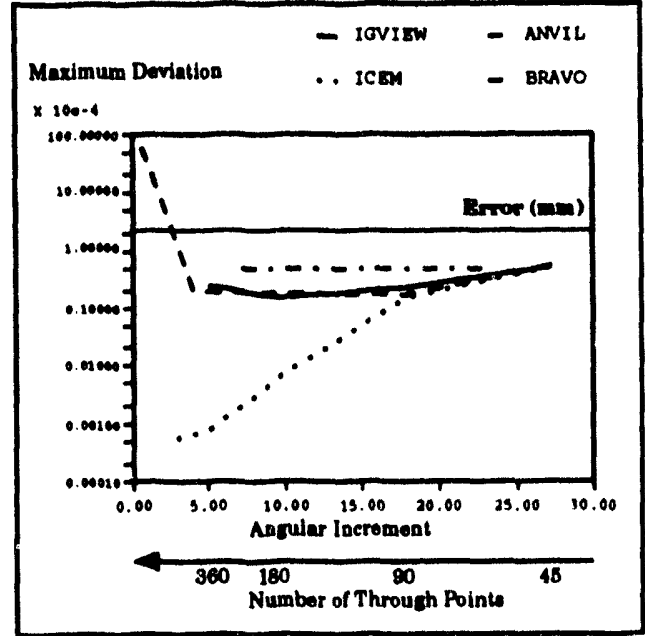

Figure 1.12. Error report for spline approximation to a $20 \mathrm{x}$ ellipse.

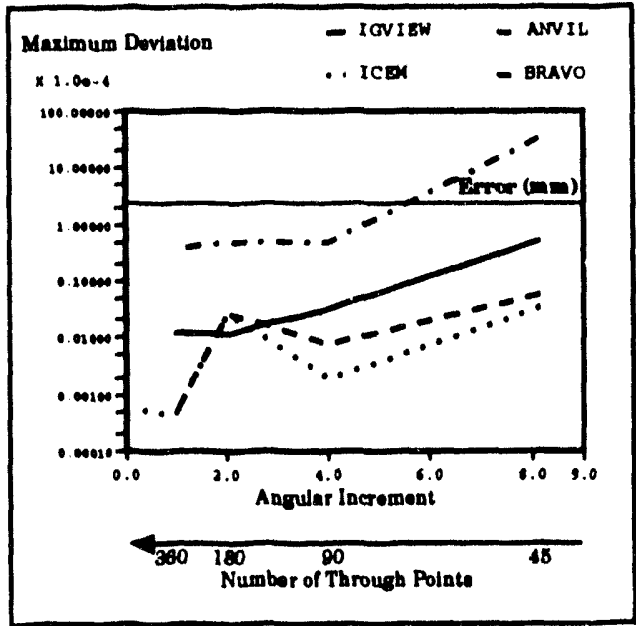

Figure 1.13. Error report for spline approximation to a sine wave.

Table 1.1 Results of CAE system tests.

\begin{tabular}{|c|c|c|c|c|c|}
\hline $\begin{array}{c}\text { CAE Module } \\
\text { Software }\end{array}$ & $\begin{array}{c}\text { Topologles } \\
\text { Modifled }\end{array}$ & $\begin{array}{c}\text { Mean Of } \\
\text { Additional } \\
\text { Points }\end{array}$ & $\begin{array}{c}\text { Std. Dev. Of } \\
\text { Additional } \\
\text { Points }\end{array}$ & $\begin{array}{c}\text { Mean Of } \\
\text { Error } \\
(\mathrm{mm})\end{array}$ & $\begin{array}{c}\text { Std. Dev. Of } \\
\text { Error } \\
(\mathrm{mm})\end{array}$ \\
\hline IGVIEW & 40 & 5.242 & 5.6325 & $1.8919 \times 10^{-4}$ & $0.4703 \times 10^{-4}$ \\
\hline BRAVO & 43 & 5.318 & 5.4155 & $1.9276 \times 10^{-4}$ & $0.4698 \times 10^{-4}$ \\
\hline ICEM & 47 & 7.015 & 6.7766 & $1.9231 \times 10^{-4}$ & $0.4851 \times 10^{-4}$ \\
\hline
\end{tabular}




\section{K. Conclusions}

The Spline Equivalence hypothesis was presented. A brief discussion of the software developed to support the hypothesis was discusses and reference were made for additional information.

Analytical and numerical tests were described that validated and verified the Spline Equivalence hypothesis. At the same time, the tests verified the numerical accuracy of the IS software.

The tests show that one set of defining point data can be used to generate equivalent topologies in different spline algorithms.

The tests also show that the number of defining points required for spline equivalence is not unreasonable.

The Spline Equivalence hypothesis presented here allows designers who have made a substantial investment in building a design data base dependent upon one CAE systems interpretation, to change to a different CAE system.

The Spline Equivalence technology also helps designers insure that their design topology is preserved when they transfer it electronically to a contractor for manufacturing. 


\section{CHAPTER 2 SOFTWARE PROJECT PLAN}

The Software Project Plan is the first of the five required Software Quality Assurance (SQA) documents. The Software Project Plan defines the project problem, goals, special considerations, project plan, and schedule.

\section{A. Problem Statement:}

The following problems have been identified with the existing spline related software:

1) The current system used to store and retrieve spline definitions (CONSYS) needs to be upgraded as follows:

a) improve storage/retrieval capabilities

b) add new contour manipulation features

c) improve interface with $\mathrm{CAE}$ systems.

2) The existing interfaces between CAE systems and CONSYS should be more automated. A new CAE package will be selected soon and will require a CONSYS interface.

3) Although the NWC has adopted Wilson-Fowler as the standard spline algorithm, various CAE packages use different spline algorithms. LANL has proposed developing software that will ensure that alternate spline algorithms will generate a spline within tolerance of the baseline definition (Wilson-Fowler).

\section{B. Project Goal:}

Build an integrated system of contour/spline-related software that consists of the following major components:

1) Storage, indexing, and retrieval of contour files

2) Interchangeable Spline (IS) software

3) Interfaces with our CAE system(s).

C. Special Considerations:

1) An interface to an expected new $C A E$ system is required by $9 / 15 / 92$.

2) Funding for this project will terminate $9 / 30 / 92$

3) The CONTOUR System will remain on VMS and does not require graphics capabilities.

\section{Proposed Plan:}

1) System requirements (to be walked through)

a) Describe current business practices.

b) General overview (how modules are to fit together).

c) CONSYS requirements (storage, indexing and retrieval).

d) CAE interface requirements. 

e) Interchangeable Spline requirements.
f) Test suites.
g) QA requirements.

2) General design (to be walked through)

a) Define interfaces among modules that must communicate

b) For each module (CONSYS, CAE interface and IS):

1) Identify and describe new software procedures and files.

2) Describe interaction among procedures.

3) Describe how software will be tested \& verified.

4) Coding and unit testing.

5) System testing and verification (to be walked through).

6) Detailed design (to be walked through) for each piece of software identified above, provide detailed specifications for the software.

\section{E. Schedule}

Table 1 outlines the tasks that the WIST Team have identified, along with target dates when each task should be completed and each task's current status. The target dates are not firm. It is difficult to estimate time requirements in any software development effort. Also, in a cross-functional (multi-group) code development effort it is difficult to predict manpower requirements and availability. It should be noted that much of the work defined in the project plan cannot be started until the new Division CAE system is purchased. This means that the project plan schedule is dependent upon when the new CAE system is selected. 
Table 2.1. Schedule for WIST team tasks.

\begin{tabular}{|c|c|l|}
\hline STATUS & $\begin{array}{c}\text { TARGET } \\
\text { DATE }\end{array}$ & \multicolumn{1}{|c|}{ TASK } \\
\hline Done & $6 / 6 / 92$ & Project Plan Draft \\
\hline Done & $6 / 15 / 92$ & $\begin{array}{l}\text { Requirements Document Draft } \\
\text { a) Describe current business practices (B. Laake) } \\
\text { b) General overview (R. Dolin ) } \\
\text { c) CONSYS requirements (B. Laake) } \\
\text { d) CAE interface requirements (K. Musgrave) } \\
\text { e) Interchangeable Spline requirements (R. Dolin) } \\
\text { f) Test suites (B. Kniss) } \\
\text { g) QA requiremerits (R. Tyler) }\end{array}$ \\
\hline Waiting & $6 / 15 / 92$ & Set date for DOE Spline Workshop II \\
\hline Working & $7 / 15 / 92$ & IS Program users manual draft \\
\hline Working & $7 / 15 / 92$ & IS Program theory manual draft \\
\hline Working & $7 / 15 / 92$ & IS Program testing and verification manual draft \\
\hline Working & $7 / 15 / 92$ & General Design document due \\
\hline Waiting & $8 / 1 / 92$ & Detailed Design document due \\
\hline Working & $8 / 15 / 92$ & Coding/Unit test completed \\
\hline Working & $8 / 15 / 92$ & Test suite completed \\
\hline Waiting & $9 / 1 / 92$ & System/QA tests completed \\
\hline Waiting & $9 / 1 / 92$ & System implemented \\
\hline
\end{tabular}




\section{CHAPTER 3 SOFTWARE REQUIREMENTS SPECIFICATION}

The Software Requirements Specification defines the Interchangeable Spline (IS) Process and the software requirements necessary to make it work. The IS process is used to generate sets of spline through points and their end conditions such that they have equivalent representations when used by different spline algorithms. The goal of the IS process is to allow the DOE Nuclear Weapons Complex (NWC) to use spline algorithms other than the Wilson-Fowler (WF) algorithm for product definition while ensuring that non-WF generated splines are equivalent to WF splines.

\section{A. History Of Spline Usage}

Spline usage has been debated within the DOE/NWC for many years. Splines are used for product definition and to drive manufacturing processes for machining and inspection.

There are many opportunities in a concept-to-stockpile evolution for spline errors to occur. Different spline algorithms may be used to interpret the same set of through-point data.

Different implementations of the same spline algorithm may be used. Different constraints, parameters, and default conditions can be applied.

Software and/or hardware incompatibilities can also allow numerical differences to occur.

The DOE/NWC uses the Wilson-Fowler (WF) spline algorithm to generate free-form geometry. WF splines are defined using a set of through-points, end conditions, and a standard WF algorithm. Once a product is designed and fabricated it is crucial to have a record of its free-form geometry (i.e., splines). It is also crucial to have a record of what components were designed and fabricated with each spline.

The WX Contour System (CONSYS) was created in the late seventies to address some of these concerns.

CONSYS is a text-based system that stores sets of through-point data and other relevant spline information. Some of the relevant information includes: name of the originating engineer, system spline was used on, date of creation, last date the spline data was accessed, and whether the spline was used to define an inner or outer surface. CONSYS provides immediate access to all spline data that has been used since its inception.

\section{Problem Statement}

Splines are used to approximate a set of through-point data with a continuous curve. There are potentially many different curves that can be approximated from a given through-point set. For example, some spline algorithms generate curves that pass through the through-points while others do not. Some spline algorithms may approximate a given through-point interval with a convex curve, while 
other spline algorithms may approximate the same through-point interval with a concave curve.

While differences can occur among various spline approximations of the same through-point data, all spline algorithms share the same underlying mathematical concept - minimizing energy. Spline algorithms take a given set of through-point data and attempt to construct a minimum energy curve. The curve between through-point pairs can vary with different spline algorithms. As more through-points are added to a spline definition, the through-points become closer and the variation between curves is reduced.

Most spline algorithms use higher order polynomials to approximate geometry between through-point pairs (e.g., cubic splines). This allows robust splines with a relatively small set of through-points. As more through-points are added, the order of the spline can be reduced. In the limit, enough through-points could be defined so that a linear (point-to-point) approximation would yield a reasonable spline definition.

This is akin to methods that CAE systems use to display circles. In general CAE systems display circles by connecting a series of points computed to lie along a circle with straight lines. The more points used to define a circle, the more the straight line segments capture the circle's characteristics. While every point along a circle is known, there is a practical limit on how many points to compute. In the case of circle display, that practical limit is determined by a CAE system's resolution. In the case of spline representation, the practical limit would be a compromise between many competing factors.

Most spline algorithms fit through-points with a certain degree of continuity. Cubic Wilson-Fowler (WF) splines are second order continuous. This means a WF spline approximation is continuous in position, slope (first derivative) and curvature (second derivative). A B-spline is generally (n-1)-order continuous. A cubic B-spline $(n=3)$, is second order continuous. The Automatically Programmed Tools (APT) software used in manufacturing and inspection processes by the NWC can compute positions, slopes, and curvatures. APT has an integrated WF spline algorithm.

Hosts of different spline algorithms are available. Most spline algorithms are superior to other spline algorithms in at least some aspect of spline representation and manipulation. Within WX-Division the Wilson-Fowler spline and the B-spline family of splines are of greatest interest. The WF spline represents the Division's current spline implementation. B-splines are becoming an industry standard and thus represent the direction that the Division should be aiming its long-term spline objectives. The B-spline family of splines includes rational B-splines, non-rational B-splines, and non-uniform rational B-splines (NURBS).

WX-Division is in the process of establishing a new CAE system standard. This new standard does not currently support WF splines. Additionally, the WF spline is a two-dimensional algorithm. This means that WF splines cannot be utilized with solid modeling technology. When a B-spline based CAE system 
becomes the WX-Division standard, potential problems arise concerning product definition, data transfer, manufacturing and inspection. The foremost problem deals with how multiple spline algorithms can be used with the same set of through-point data to achieve equivalent product definition. Another significant problem with using non-WF CAE systems is maintairing a historical product definition.

\section{Proposed Solution}

A solution to these problems is to develop a process that allows multiple spline algorithms to be used with a given through-point set of data to produce an equivalent WF spline representation. This process ensures that a CAE system spline and a WF spline are equivalent at the through-points and intermediate points. Equivalence is defined as being within a predefined tolerance band. A result of this process is a spline that is interchangeable with the DOE standard WF spline and the CAE system used for comparison.

a) Interchangeable Spline Process. The interchangeable spline (IS) process in WX-Division contains three functional modules; a CAE System module, a Spline Equivalence (SE) module and a spline data base module. A Process Contoller (PC) automates the IS process. The PC is used to interact between the CAE system module and the $\mathrm{SE}$ module. Additionally, the PC is used io interact between the $\mathrm{SE}$ module and the spline data base module. It is the PC's responsibility to integrate the $\mathrm{CAE}$ system module and the spline data base module into the SE module. The spline data base module is not required to make the IS process work. However, it is an attribute of the WX-Division implementation.

b) Spline Equivalence Module. The SE module contains an integrated WF spline algorithm that is used to generate baseline WF spline representations. The SE module will be developed as a stand alone program. In other words, it will not be dependent upon the CAE system module or the spline data base module. This gives the SE module an ability to be shared by DOE/NWC agencies without having to share the same CAE system or spline data base module. The SE module can stand alone because module interactions are handled by a process controller and data exchange between modules is done with file transfers. The $\mathrm{SE}$ module reads and writes files, so to use it one only needs to understand what the I/O files look like and how to access them.

The SE module's only capability is to process splines and make them interchangeable between a baseline spline and another spline algorithm. In one case the WF spline is the baseline and the CAE spline is made equivalent to it. In another case, the CAE spline is the baseline and the WF spline is made equivalent. The SE module ensures that a spline is within a specified tolerance of the baseline representation. The impetus for the spline equivalence capability is that once implemented each NWC agency is free to use whatever spline algorithm they prefer, provided each spline is processed using the SE module.

c) Spline Data Base Module. WX-Division has developed a utility to store, retrieve and manipulate contours, 
called CONSYS. Contours do not contain end angles and so are not splines. It is necessary to overhaul CONSYS to keep pace with evolving CAE software and operating system technologies. The new CONSYS will initially be developed on the VAX/VMS operating system and may later be port d to the Division's UNIX platform. The new CONSYS will be developed in multiple phases. In later phases the existing CONSYS functionality will be preserved in a new data base structure. In phase two, new spline manipulation and data base features will be added. The new CONSYS will be written in double precision FORTRAN and called the WFSYS. Throughout the remainder of this document references to the WFSYS module represent an optional spline data base that is not required to implement the IS Process.

d) CAE System Module. The CAE system will input spline data in an IGES format. The CAE system's internal spline algorithm will generate a spline using the information obtained from the input file. The CAE system will output a full IGES representation of its computed spline (through-points, coefficients, and parameterization). The IS process is not dependent upon any particular $C A E$ system, only the required CAE system functionality.

e) Spline Usage Document. In order to maintain design integrity, spline usage procedures must be documented. A spline usage procedures document will be used in conjunction with the IS-process to generate interchangeable splines that can be used for product definition. The procedures are simply a set of rules that govern what can and cannot be done with splines during product development. Spline usage procedures will be concurrently developed with the new WFSYS module.

f) Test/Verification. Each time a new version of the CAE system or the SE module is introduced, the entire spline-related software system must be tested and verified by the procedures specified by the quality assurance requirements. There will be a suite of test problems that will be used to evaluate each new software component. The test suite will include deflavored design contours and analytical curves.

g) Quality Assurance. The entire spline-related software system is being developed as a cross-functional team effort. A quality assurance plan will be specified to assure total quality at each phase of development. The quality assurance plan defines how software will be designed, developed, tested, and documented. 


\section{B: INTERCHANGEABLE SPLINE PROCESS}

The four components of the IS process are shown in figure 3.1. From a user's perspective, the IS process can be initiated from either the WFSYS module or the CAE system module. The IS process is initiated from the WFSYS module to verify that splines can be made interchangeable and also when spline data is extracted from WFSYS. The IS process is initiated from the CAE system module to get new splines into the CAE system's data base and to process an existing CAE system spline that has been modified (e.g., trimmed, offset, etc.).

The IS process exchanges information between the three modules by reading and writing files. There are four files used by the IS process: WF2SE.DAT, SE2WF.DAT, CAE2SE.IGS, and SE2CAE.IGS. The two ".DAT" files exchange information between the SE module and the WFSYS module. The two ".IGS" files exchange information between the SE module and the CAE system module. File WF2SE.DAT is written by the WFSYS module and read by the SE module. The file is an ASCII text file that contains a spline's through-points and end angles. File SE2WF.DAT is written by the SE module and read by the WFSYS module. This file contains the through-points and end angles for a processed interchangeable spline.

The file CAE2SE.IGS is written by the CAE system and read by the SE module. This file is in an ASCII IGES format that contains a full spline representation (through points, coefficients, and parameterization). The IS process supports several IGES spline types including the 112 and 126 spline entities. The file SE2CAE.IGS is an ASCII IGES formatted file written by the SE module and read by the CAE system module.

\section{Function Definitions}

The purpose of this section is to provide an overview of how each component of the IS process operates. This overview describes functionality. A discussion of the IS process design and operating flow charts follows.

a) Spline Equivalence Module Functional Definition. The SE

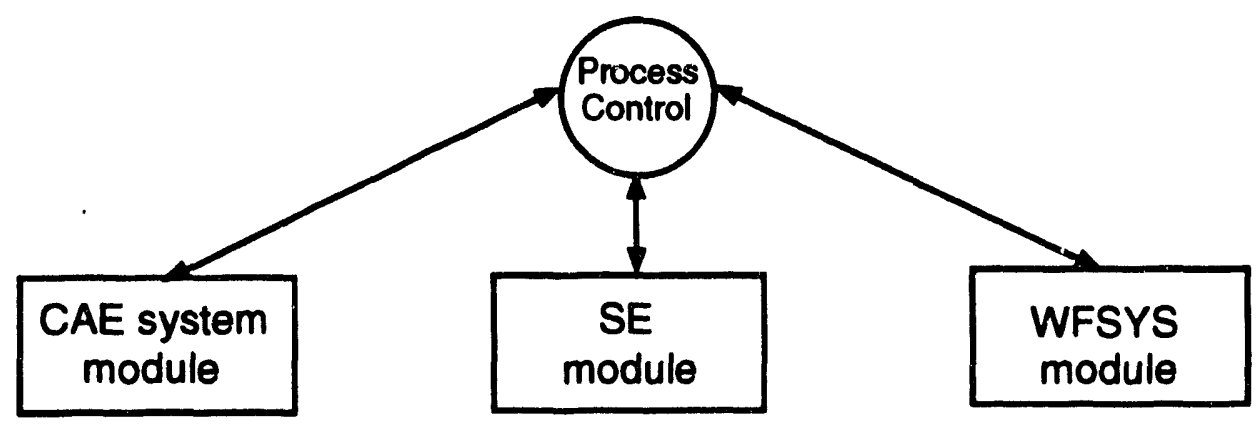

Figure 3.1. The four independent components of the IS process. 
module compares a WF spline with a CAE system spline and modifies the through point information of one spline until it is within a specified tolerance of the other spline (i.e., equivalent). The SE module's output is an interchangeable spline that is represented as a set of through-points and end angles in file SE2WF.DAT and as an IGES file in CAE2SE.IGS.

b) SE Module When Processing A WF spline. When a WF spline is processed into an interchangeable spline, the SE module begins by reading the spline data contained in file WF2SE.DAT and computing a WF-baseline spline. The SE module then writes file SE2CAE.IGS and waits for the CAE system to compute its version of the spline. A process controller is used to direct the interaction between the SE module and CAE system. When the CAE system has completed its tasks, the SE module reads the CAE spline from file CAE2SE.IGS. The SE module then begins an iterative process of comparing the CAE spline with the WF-baseline spline for equivalence. The CAE spline is modified by subdividing necessary intervals until the two splines are equivalent.

c) SE Module When Processing A CAE spline. When a CAE spline is processed into an interchangeable spline, the SE module begins by reading the spline data contained in file CAE2SE.IGS. This CAE spline becomes the baseline spline for the equivalence process. The through-points are extracted from the baseline spline representation and used to generate a WF spline. The two splines are checked for equivalence. If they are not equivalent, the WF spline definition is modified by subdividing necessary intervals until the two splines are equivalent. Interaction between the SE module and CAE system is handled by a Process Controller.

\section{d) CAE System Module}

Functional Definition. There are three functional modes of operation for the CAE system module. The CAE system can initiate the IS process when a new spline is desired from WFSYS or when an existing spline needs to be put into WFSYS. The CAE system is also executed from within the SE module.

The CAE system is executed by the SE module when the SE module needs a CAE spline representation of a set of through-point data. In this mode, the CAE system reads file SE2CAE.IGS, generates a CAE spline and outputs that spline in file CAE2SE.IGS. In all cases, a Process Controller handles module interactions and data flow.

e) WFSYS Module Functional Definition. Functionally, the WFSYS module reads, writes, retrieves, and modifies WF splines. Splines are stored in WFSYS as through-point coordinate data and end angles. WFSYS will perform WF spline manipulations by integrating the SE module's WF spline algorithms. When a spline is requested by the IS-process, WFSYS retrieves the spline and writes it in file WF2SE.DAT. When an interchangeable spline has been generated by the SE module, WFSYS can retrieve it from file SE2WF.DAT. A process controller directs the information flow.

WFSYS can be run interactively, independent of the IS process (or the IS process can be thought of as a command option within WFSYS). WFSYS can 
perform many spline manipulation features. Since interchangeable splines are not stored in WFSYS, all spline data extracted from WFSYS must be run through the IS process to ensure interchangeability.

f) Process Control Functional Definition. Details of the Process Controllers have intentionally been left nebulous. This is because it is too early to be concerned with the exact design of the PCs. This section discusses the functional design of the PCs. Their details can be specified in subsequent documents. The process controllers are hardware and software dependent.

\section{g) Process Controller Executed From Within SE module}

The SE module needs to interact with the CAE system module. The tasks of the Process Controller when executed within the SE module are

Execute the CAE system

(1) Read file SE2CAE.IGS - Generates CAE spline

(2) Write CAE2SE.IGS

(3) Return control to SE module

\section{h) Process Controller Executed Within WFSYS}

The WFSYS module has many spline manipulation features. A user must process splines when extracted from WFSYS and should process splines after modifications to ensure that the modified spline is interchangeable. When a user in WFSYS requests that a spline be processed, WFSYS writes the user-specified spline to file WF2SE.DAT and executes a process controller. The process controller then

(1) Runs SE module - Passes information to SE module indicating that a WF spline is being processed so that the SE module knows to make the WF spline its baseline

(2) Returns control to WFSYS 


\section{1) Process Controller Executed When CAE gystem Requests A WFSYS Spline}

Users of the CAE system will often find it necessary to pull a spline into their CAE data base from WFSYS. In this mode of operation, the CAE system user requests a spline be pulled in from WFSYS. The CAE system executes a process controller and passes on the requested spline name. The PC then

\section{Runs WFSYS}
a) Reads spline name
b) Writes spline file WF2SE.DAT

2. Runs SE module - Passes information to SE module indicating that a WF spline is being processed so that the SE module knows to make the WF spline its baseline.

3. Informs CAE system user that file SE2CAE.IGS is available

4. Quits

\section{j) Process Controller Executed When CAE system Requests A CAE spline Be Processed}

CAE system users must process new or modified splines before releasing product definitions. A CAE system model represents product definition. This means that once a WFSYS spline has been modified in the CAE system,. the original spline no longer represents product definition. The new spline, that represents product definition, must be processed to; ensure that it can be made interchangeable, get a new WFSYS name and store it in WFSYS as a temporary spline. To process a new or modified spline a process controller is executed to

1. Write file CAE2SE.IGS

2. Run SE module - Pass information to SE module indicating that a CAE spline is begin processed so that the SE module knows to make the CAE spline its baseline.

3. Run WFSYS - Store interchangeable spline as a temporary spline and determine a WFSYS name for the temporary spline.

4. Inform CAE system user that SE2CAE.IGS file is ready, and the name of interchangeable spline.

5. Quit 


\section{General Desien}

This section discusses the operating modes of the SE module. The textual and graphical flow charts define a general SE module design.

a) SE module When Processing a WF apline. The SE module can generate an interchangeable spline using a baseline WF spline. There are two ways that this process can be initiated, from WFEYS and from the CAE system. Section 2.1 discussed when each mode is used. The important thing to note about using the SE module is that it expects either the
WF2SE.DAT file or the CAE2SE.IGS for input, and outputs the SE2WF.DAT and SE2CAE.IGS files. The following textual flow chart describes the SE module in this mode.

A graphical flow chart can also be used to describe the IS-process when a WF spline is being processed. Figure 3.2 shows a graphical flow chart of the IS-process when a WF spline is being processed.

b) SE module When Processing a CAE spline. The SE module can be initiated from the CAE system when the CAE system user has modified an

\section{Spline Equivalence module execution for processing a WF spline}

1. Determine that a WF spline is being processed.

2. Read file WFaSE.DAT into baseline spline arrays.

3. Store through-points in temporary spline arrays.

4. Generate baseline WF spline.

5. Write file SE2CAE.IGS using temporary spline array data.

6. Spawn a process to execute CAE system.

a. Read file SE2CAE.IGS

b. Generate CAE spline.

c. Write CAE spline in file CAE2SE.IGS.

d. Exit CAE system.

7. Compare CAE spline with baseline WF spline for equivalence.

8. If splines are equivalent,

a. Generate WF spline using temporary spline definition.

b. Compare temporary WF spline with baseline WF spline.

(1) If splines are not equivalent -- ERROR -- STOP.

(2) If splines are equivalent continue.

c. Write file SE2WF.DAT.

d. End SE module operation.

9. If splines are not equivalent

a. Add through-points in intervals where splines are not equivalent.

b. Store modified spline definition in modified spline arrays.

c. Write modified spline to file SE2CAE.IGS.

d. Repeat from step 6. 
existing spline or has generated a new spline. In either case, the user wants to ensure that the current spline can be made interchangeable, acquire a new WFSPL name, and temporarily store the interchangeable spline in WFSPL. The following textual flow chart describes the SE module when a CAE spline is being processed. In this mode, the SE module expects a file called CAE2SE.IGS to be available to input.

A graphical flow chart can also be used to describe the IS-process when a CAE spline is being processed as shown in figure 3.3.

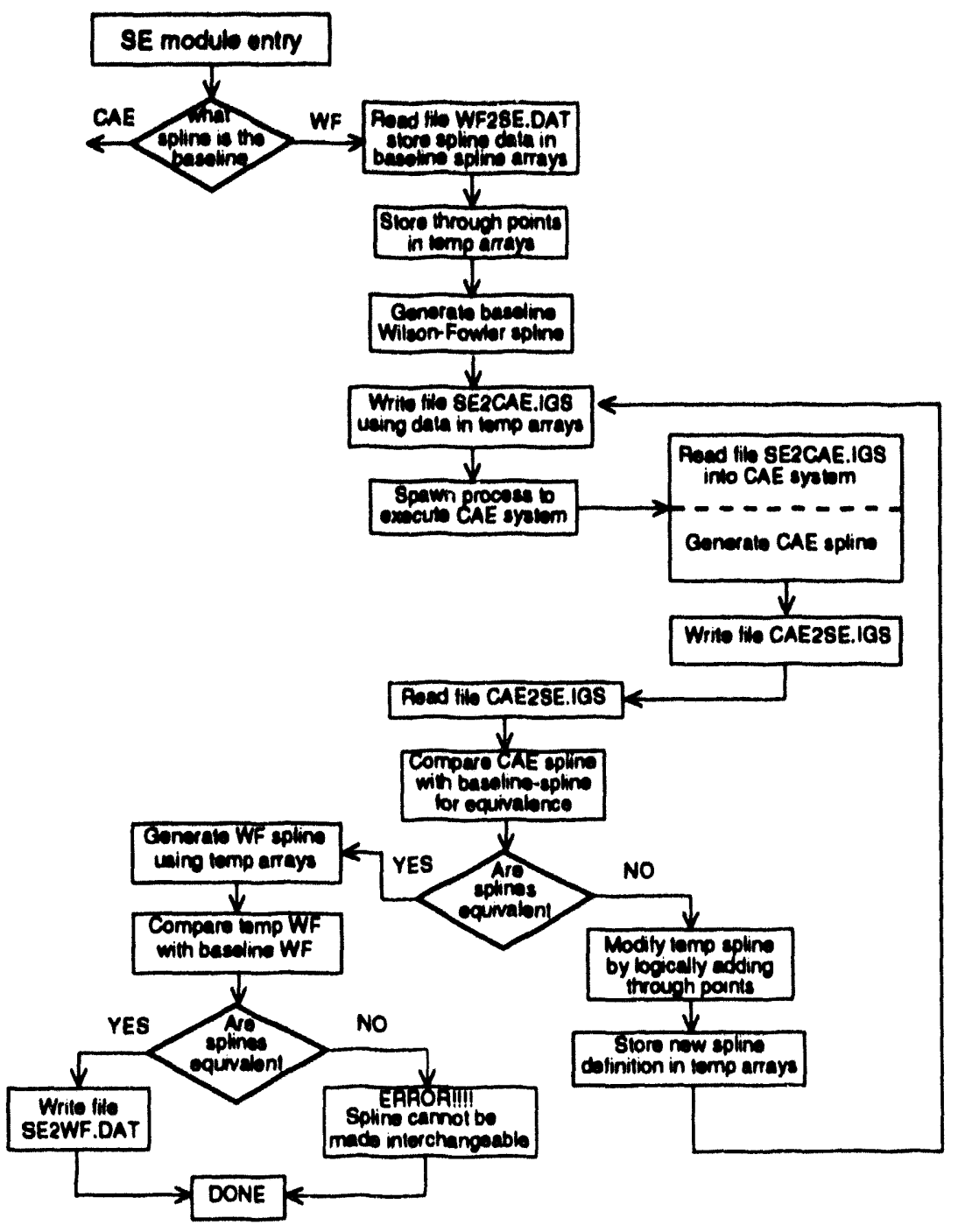

Figure 3.2. The IS process when a WF spline is being processed. 


\section{Spline Equilvalence module execution for CAE syatem spline}

1. Determine that a CAE spline is being processed.

2. Read file CAE2SE.IGS into baseline spline arrays.

3. Store through-points in temporary spline arrays.

4. Generate WF spline.

5. Compare WF spline with baseline CAE epline for equivalence.

6. If splines are equivalent,

a. Write temporary spline in file SE2CAE.IGS.

b. Spawn a process to execute CAE system.

(1) Read file SE2CAE.IGS.

(2) Generate CAE spline.

(3) Write CAE spline in file CAE2SE.IGS.

(4) Return control back to SE module.

c. Read CAE2SE.IGS file,

d. Compare temporary CAE spline with baseline CAE spline.

(1) If splines are not equivalent -- ERROR -- STOP.

(2) If splines are equivalent, continue.

e. Write file SE2WF.DAT.

f. End SE module operation.

7. If splines are not equivalent

a. Add through-points in intervals where splines are not equivalent.

b. Store modified spline definition in temporary spline arrays.

c. Repeat from step 4.

\section{Spline Equivalence Module Requirements}

The SE module reads and writes XY. and IGES-formatted ASCII files. The SE module assumes that splines originate as either an IGES file written by a CAE system or as a file of XY data.

\section{Languages And Platforms}

The Spline Equivalence module will be written in prodominately in FORTRAN. There will be some subroutines that can accomplish their tasks more efficiently using the $C$ programming language.

Mixing FORTRAN with $\mathrm{C}$ is not a problem. However, in order to use the software, users will need access to both types of compliers.

The FORTRAN and $C$ subroutines will be physically kept separate 80 that users will be able to identify them.

The SE module will be developed on the VAX/VMS platform and ported to other platforms. Specifically, to UNIX platforms. 


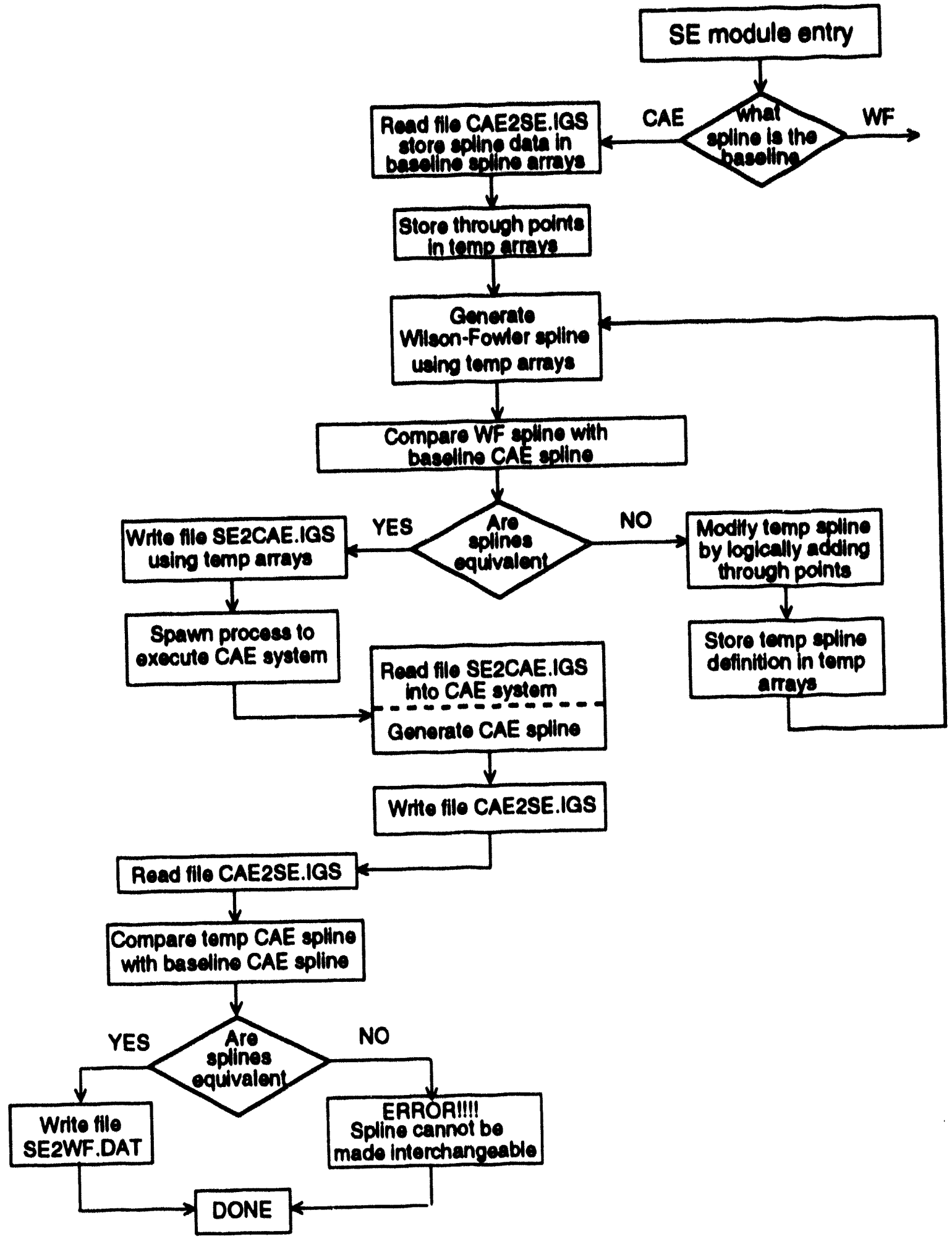

Figure 3.3. The IS process when a CAE spline is being processed. 


\section{Modules of Spline Equivalance Program}

Main Module - directs the SE processes.

$$
\begin{aligned}
& \text { Input/Output Module } \text { - Read file WF2SE.DAT (XY-data file) } \\
& \text { - Write file SE2WF.DAT (XY-data file) } \\
& \text { - Read file CAE2SE.IGS (IGES CAE spline) } \\
& \text { - Write file SE2CAE.IGS (IGES file) }
\end{aligned}
$$

Wilcon-Fowler Spline Module - Stand-alone WF spline program.

Evaluation Module - Splines are evaluated against baseline, and logic controls through-point additions.

Interface Module - System dependent code. Each agency is responsible for the development of the appropriate code for this module.

The SE module will be written in modular form based on functionality.

2. Documentation, Quality Aseurance, and Training

The SE module's necessary documents, such as the user manual and Software Quality Assurance (SQA) documents will be written as specified by the SQA procedures. The SQA processes that will be used is outlined in section $\mathbf{G}$.

User training will begin after implementation and beta testing have been completed.

\section{Baseline Product Definition}

The CAMI-APT Wilson-Fowler spline algorithm will be the standard or baseline spline algorithm used by the Spline Equivalance program. ${ }^{1,2}$

Each NWC agency is responsible for ensuring that its splines are equivalent to the standard definition.

\section{Multiple Spline Representation}

Multiple spline representations will be supported by evaluating the deviation of each representation from the standard definition. An interchangeable spline developed between one CAE system and the SE module, may be numerically different than an interchangeable spline developed between another CAE system and the SE module, but they will be geometrically equivalent.

1 R. M., Dolin. "The Wilson-Fowler Spline In A Global IGES Coordinate Frame," Los Alamos Nationul Laboratory Repon Number LA-1 1024-MS, (September, 1987).

2 R. M., Dolin, "A Reference Guide For IGVIEW, An IGES.Compatible Geometry System," Los Alamos National Laboratory Report Number LA-11025-MS, (October, 1987). 
NWC agencies may design, manufacture, and inspect parts using their interchangeable spline representation.

\section{b. Data Exchange}

Data exchanged between the SE module and a CAE system will be via the IGES data exchange format.

Data exchange between the NWC agencies will be via the IGES data exchange format.

\section{Geometry Constrainte}

No embedded linear segments will be allowed.

Splines will have defined end conditions or the WF defaults will be used.

The maximum number of data points used to define an interchangeable spline will be determined by a site survey of existing software limitations.

The direction of the spline is implicitly defined by the data set.

\section{Software Access}

Los Alamos National Laboratory will share QA'ed versions of the SE module software. They will develop a spline procedures document.

Each agency is responsible for checking the shared software implementation against in-house implementation.

Los Alamos National Laboratory is responsible for coordinating the selection or development and QA process of shared software.

- SE module software

- Wilson-Fowler spline software

- Documentation and source code
- Test suites

\section{Convercence Measures}

An interchangeable spline will be compared to the baseline spline at $\mathbf{N}$ evenly spaced points along the baseline spline $(N \sim 1000)$. Local deviation is measured as the distance from one of the $\mathrm{N}$-baseline evaluation points to the closest point on the interchangeable spline.

Initially, the maximum acceptable level of deviation from the baseline spline is proposed as $0.00025 \mathrm{~mm}$ for the design agencies.

\section{Interchangeable Spline Program}

Initially, points will be added to an interchangeable spline by logically subdividing through-point intervals. Original points will not be moved.

\section{SPLINE DATA BASE MODULE REQUIREMENTS}

A spline data base is not required to run the IS-process. WX-Division has historically used a contour data base for product definition (CONSYS), but is evolving to a Wilson-Fowler spline data base for product definition (WFSYS).

The intent of this spline data base evolution is to enhance current capabilities, add features, and improve compatibility with new CAE environments currently being implemented in the NWC. The new spline data base must not compromise the integrity of the contour data currently stored in CONSYS.

The migration of CONSYS data to WFSYS will be done in multiple phases. Phase one will revise CONSYS on VAXVMS. The phase one WFSYS will 
increase the number of allowable data points to 1000 , store end angles, and add one new command that generates an interchangeable spline. This new command will allow users to specify end conditions or will compute the WF default end conditions.

Phase two will develop WFSYS on UNIX. Migration of CONSYS data to WFSYS will be performed manually as spline data is requested. The enhanced features that will be available in this phase include:

1. Parent/child relationship

2. Coordinate data - Through-point data and end angles in double precision

3. Application - Where was spline used

Future enhancements include spline manipulation features. Other enhancements not specified will be evaluated on their merits.

\section{E. CAE INTERFACE REQUIREMENTS}

An interface is needed to allow the exchange of data between the Division's primary CAE system and the IS process. This interface allows interchangeable splines to be read into the CAE system. It will also allow CAE system generated splines to be processed into interchangeable splines. Interfaces to other CAE systems used within the Division will be evaluated on their merits. Other NWC agencies will be responsible for their own CAE system interfaces.

\section{Languages And Platforms}

The Division's primary CAE system will reside on UNIX. The CAE system's graphics programming language will be used as an interface between the CAE system and the IS process.

\section{Mode Of Operation}

Iterations with the IS process will be performed in batch mode. The user requesting an interchangeable spline will be notified when processing is complete and will then be allowed to read the interchangeable spline into the CAE system.

\section{Data Exchange}

The data exchange format and methodology is discussed in section B.

\section{F. TEST/VERIFICATION PLAN REQUIREMENTS}

Each time that a new version of the CAE system or the SE module is introduced, the entire spline related software system must be tested and verified by the procedures specified by the Quality Assurance requirements. There will be a suite of seven test problems that will be used to evaluate each new software component. The seven test problems will be comprised of five deflavored contours and two analytical curves.

The five deflavored contours will be taken from existing product definition. They are meant to represent actual design geometry. The two analytical curves are included in the test suite because higher ordered information is available. Analytical curves also provide exact coordinate positions at any intermediate point.
G. QUALITY ASSURANCE REQUIREMENTS 
The proposed Software Quality Assurance (SQA) procedures for the IS process project are listed below. This plan follows the SQA plan defined by the Department of Energy (DOE) for all software that is to be shared by outside agencies.

\section{Software Project Plan (SPP)}

The SPP will initially be reviewed and approved by the WIST Team and subsequently reviewed and approved by WX-Division management. The SPP is a short document establishing the scope of the project, the project goals (deliverables), restrictions, and assumptions. The SPP document identifies major az eas of responsibility.

\section{Software Requirements Specifications (SRS)}

There are two parts to the SRS. Part 1 documents system requirements. Part 2 defines the general design of the system that satisfies these requirements. The design identifies all files to be used or created by the system and all programs and major functions that comprise the system. A block diagram with a few lines of description on files and programs is adequate.

The SRS document will first be approved by the WIST Team and then given to WX-Division management for final review and approval. The SRS document will next be given to the DOE Spline Committee for review and approval.

\section{Software Design Description (SDD)}

The SDD document contains detailed record descriptions for files identified in the SRS, final report layouts (if any), and final screen designs. The level of detail of the SDD document is such that a knowledgeable person could generate code from it with little or no verbal instructions. The SDD document need only be reviewed and approved by the WIST Team.

\section{Software Veriflcation and Validation Plan (SVVP)}

The SVVP is a narrative of how the software is to be tested. The SVVP document is nothing more than a checklist to identify what is to be tested and under what conditions. Software verification and validation should be approached like an experiment. All software changes are stopped at this point. The SDD document need only be reviewed and approved by the WIST Team.

\section{Software Verification and Validation Report (SVVR)}

The SVVR documents the results of the testing process identified in the SVVP. The SVVR may be as simple as a lab notebook recording each step of the testing process. If the software fails to perform a test successfully the shortcomings of the software are identified in the SVVR document and corrected. The tests are repeated and the results are recorded in another SVVR. 


\section{CHAPTER 4 SOFTWARE DESIGN DESCRIPTION}

The Software Design Description describes the program subroutines used by the Interchangeable Spline system. The subroutines are grouped into functional modules.

The primary function of the Interchangeable Spline (IS) program is to take a set of coordinate data (XY), and insure that an integrated Wilson-Fowler (WF) spline algorithm and a user specified spline algorithm are equivalent.

Equivalence is defined by the variable EQIV_TOL. To converge, the spline intervals that exceed EQIV_TOL are subdivided.

Error is computed as the linear deviation from a baseline spline to the approximating spline. Both splines are evaluated using their IGES parametric representations. The baseline spline is used to compute the locations for new through-points. The midpoints between two existing through points is taken as one-half of the interval's parametric arc length.

Spline data can be read into the IS process as either ASCII XY data or as a CAE-IGES data. When ASCII XY data is initially read into the IS process, the integrated WF spline is the baseline spline. When a CAE-IGES is initially read, the CAE spline becomes the baseline spline.

If ASCII XY data is initially read: The data will be in the form of an ASCII text file of NPTS +2 records. The first record contains the integer value for the number of through points to be read (NPTS). The next NPTS records contain the $\mathrm{XY}$ coordinates of the spline's through-points and the NPTS+2nd record contains the spline's end angle information.

The GET_WF algorithm assumes that a file called "XY2IS.DAT" exists in the current working directory. That file is assumed to be in the format described above.

Next, the baseline spline is generated (baseline definition is the Wilson- Fowler spline in this mode). The baseline spline is a WF of the following format:

$\mathrm{X}=\mathrm{AX} * \mathrm{~s}^{3}+\mathrm{BX}^{*} \mathrm{~s}^{2}+\mathrm{CX} * \mathrm{~s}+\mathrm{DX}$ $\mathrm{Y}=\mathrm{AY} \mathrm{s}^{3}+\mathrm{BY} \mathrm{s}^{2}+\mathrm{CY} * \mathrm{~s}+\mathrm{DY}$

The parametric form for a coordinate position within the Ith interval, in terms of the software array syntax, is:

$$
\begin{aligned}
& \mathrm{X}(\mathrm{I})= \begin{array}{l}
\operatorname{BLSPL}(2, \mathrm{I}) * \mathrm{~s}^{* *} 3+\operatorname{BLSPL}(3, \mathrm{I})^{*} \mathrm{~s}^{* *} 2+ \\
\operatorname{BLSPL}(4, \mathrm{I})^{*} \mathrm{~s}+\operatorname{BLSPL}(5, \mathrm{I})
\end{array} \\
& \mathrm{Y}(\mathrm{I})= \begin{array}{l}
\operatorname{BLSPL}(6, \mathrm{I})^{*} \mathrm{~s}^{* *} 3+\operatorname{BLSPL}(7, \mathrm{I})^{*} \mathrm{~s}^{* *} 2+ \\
\operatorname{BLSPL}(8, \mathrm{I})^{*} \mathrm{~s}+\operatorname{BLSPL}(9, \mathrm{I})
\end{array} \\
& \operatorname{BLSPL}(1, \mathrm{I})<=\mathrm{u}<=\operatorname{BLSPL}(1, \mathrm{I}+1) \\
& \mathrm{s}=\mathrm{u}-\operatorname{BLSPL}(1, \mathrm{I}) \\
& \mathrm{u}(\mathrm{I})=\mathrm{u}(\mathrm{I}-1)+\operatorname{BLSPL}(1, \mathrm{I}-1)
\end{aligned}
$$

For more information, see: R. M.,Dolin, "The Wilson-Fowler Spline In A Global IGES Coordinate Frame," Los Alamos National Laboratory Report Number LA-11024-MS, (September, 1987). 
The next step in the process is to drop into the IS driver and let it run the show until an equivalent IS spline is generated. This is done by generating both WF and CAE splines using the initial set of through point data. New points are added in the through-point intervals where errors exceed EQIV_TOL, until splines converge.

The IS software needs to determine if the process is being initiated from the WF side or the CAE system side of the SE module. In other words, if the WF or CAE system spline will be baseline.

Read baseline spline, either a WF or the CAE system spline. If you are reading a WF baseline spline, the raw $\mathrm{XY}$ data is read from file "WF2SE.DAT" and full WF spline is returned in the BL_SPL array. Drop into the SE driver and let it run the show until an equivalent IS spline is generated.

\section{A. Main Driver Module}

\section{BASELINE_GEN(BL_SPL, NBLPTS, ANGIN, ANGOUT, IAFG)}

This subroutine generates a baseline Wilson-Fowler spline using $X Y$-coordinates and end angle information. The output from this routine is an array of a full IGES cubic WF spline definition and an array of the segment lengths of each interval.

Generate an IGES WF definition of the spline using the XY-coordinates of the through-points and the end angle information. Variables in this subroutine are:

INPUT PARAMETERS:

BL_SPL = Baseline spline's coordinate data

NBLPTS = Number of through-points .
IAFLG $=\underset{\text { Angle flag }=1 \text { if end angles }}{\text { exist }}$

OUTPUT PARAMETERS:

ANGIN = End angle at the start point of the spline

ANGOUT $=$ End angle at the last point of the spline

\section{DIRECTORY}

This subroutine contains a listing of all of the modules within the IS program, and all of the subroutines within each module. Along with each entry is a brief description of what its main function is.

\section{VARIABLES}

This subroutine contains a listing of all of the variables within the IS program. The variables are organized into real, integer, and character. Along with each variable is a description of what the variable is used for and a pointer to the subroutine where it is used (when that makes sense to do). The format for this subroutine is one big write statement.

\section{B. IS_EVALUATION MODULE}

This module analyzes through point and intermediate point data (coordinates) for two spline representations. The algorithms compare Wilson-Fowler (WF) splines with CAE system generated splines. The WF spline algorithm is integrated into IS software and the CAE system splines are imported via the IGES file transfer protocol. The integrated WF spline is the APT WF spline program.

The splines are evaluated at MXKNOTS-NKNOTS evenly spaced 
parametric points. Since the defining through-points may not fall on one of the evenly spaced parametric evaluation points, they are evaluated as well. By definition, the splines are supposed to pass through the points, but you will check just to be sure. There is a predefined tolerance (TOL) that is used to measure when the two splines are close-enough. The evaluation is done by computing the coordinates of the WF spline at a given parametric point and then finding the nearest point on the CAE spline.

Since the form of parameterization is probably different for the two splines, you cannot simply evaluate each spline at a given parametric location. If the two splines are within TOL at every parametric evaluation point, the splines are said to have converged. If they are not within TOL at every evaluation point, the through-point intervals where errors occurred are subdivided and the modified spline is re-evaluated.

The WF spline algorithm is used to compute new through-point coordinates on the modified spline. The evaluation-subdivision loop continues until the two splines have converged.

In theory, the splines will always converge. When a new converged spline definition is generated, the new WF spline is compared with the original WF spline. This is done to insure that as new points are added to the spline, it still converges with the original spline definition. This is a very important feature of the interchangeable spline software algorithm and will be discussed in more detail in one the subroutines of this chapter.
Since the WF spline algorithms are integrated into the IS software, it is easy to compute modified WF splines. The CAE system must be assessed in order to compute modified CAE splines. This requires spawned processes.

1. EVALUATE (SPLTYP, BL_SPL, NBLPTS, ANGIN, ANGOUT, TMP_SPL, NTPTS)

INPUT PARAMETERS:

SPLTYP - Flag specifying the spline type of the baseline spline

BL_SPL - Baseline spline definition

NBLPTS - Number of through points in the baseline spline definition

ANGIN - Entry angle for WF spline ANGOUT - Exit angle for WF spline

OUTPUT PARAMETERS:

TMP_SPL - Final version of modified spline after it has been made equivalent to the baseline

NTPTS - Number of points in the final modified spline

The algorithm manages the process of comparing two different spline algorithms and doing whatever it takes to make them equivalent; in this case, requiring that in each interval for which the splines differ by more than tolerance be subdivided. The baseline spline is used to determine the coordinates of the subdivision point. The new set of knot points is used to compare a new WF and CAE spline for equivalence.

If this process was initiated from the WF spline side of the SE module, then the first step you do is compute a CAE 
spline. So, write the CAE spline file and spawn a process to interface with the CAE system. Compute the CAE version of the current spline and output an IGES representation of it. When the spawned process is finished, read the CAE spline into the temporary arrays and begin the equivalence processing.

If the process began on the CAE side of the SE module, then you have to use the through points of the CAE spline (the baseline in this scenario) to build a WF spline. Once a WF spline has been generated, the equivalence process can begin.

If the error flag is false, the temporary spline has converged. Take the newly specified set of temporary through points and generate a temporary spline definition. Then compare the new temporary spline with the baseline spline for equivalence.

\section{EQUIVALENCE (BL_SPL, NBLPTS, SPLTYP, TMP_SPL, NTPTS, ERR)}

INPUT PARAMETERS:

BL_SPL - Baseline spline array of defining coefficients

NBLPTS - Number of baseline spline through points.

SPLTYP - The type of baseline spline that is being used.

OUTPUT PARAMIETERS:

TMP_SPL - Array defining the temporary splines defining points.

NTPTS - Number of points in the temporary spline.
ERR - Largest error between baseline spline and temporary spline.

This subroutine compares the baseline spline with the temporary spline for equivalence. If the splines are not equivalent, subdivide the intervals of the temporary spline where equivalence is not met.

The logical 'ERR' is used to specify when convergence is meet. ERR will be false when the splines are equivalent and true otherwise.

Evaluate "nevals" points evenly spaced within each spline interval. Include the end points. The parameter " 8 " varies for each interval. Start point of each interval is evaluated, but end point may not be.

Assume that both splines are monotonic (i.e., intervals do not loop back on each other). The temporary spline will be stepped through, and the nearest point on the baseline spline will be found. The first step in this process is to compute a tangent vector to the temporary spline at the current evaluation point. Then, use the Serrate-Frenet equations and binormal to compute a normal vector.

Project that normal vector through the baseline line and compute the intersection point. This may require solving a cubic equation for its three roots or using numerical equations such as Horner's method. The first interval that contains an error is subdivided in its center when done.

The first thing this algorithm does inside the 250 Do-Loop is to compute the tangent along the temporary spline. The tangent is determined as a vector from the last evaluation point to the 
current evaluation point (unless you are evaluating the first point).

Once the tangent is known, you can use it along with the binormal to compute the normal vector. For a 2D curve, the binormal is simply a vector in the Z-direction. The Serret-Frenet equation for a normal to a curve is:

Normal $=$ Binormal crossed with the Tangent (N = B X T)

The equations for the various vectors are:

where

$$
T=A e_{1}+B e_{2}+\widetilde{C} s_{3}
$$

$$
\begin{aligned}
\mathbf{C} & =0 \text { for } 2 \mathrm{D} \\
\mathrm{A} & =\mathrm{X} 0+\mathrm{a}^{*} \mathrm{t} \\
& =[\mathrm{Px}(\mathrm{i}-1)-\mathrm{Px}(\mathrm{i})]^{*} \mathrm{t} \\
\mathbf{B} & =\mathrm{Y} 0+\mathrm{b}^{*} \mathrm{t} \\
& =\mathrm{Py}(\mathrm{i})+[\mathrm{Py}(\mathrm{i}-1)-\mathrm{Py}(\mathrm{i})]^{*} \mathrm{t} \\
& =1 \mathrm{e} 3
\end{aligned}
$$

The binormal unit vector in the Z-direction is:

$$
\mathrm{RN}=\mathrm{BXT}=-\mathrm{B} \mathrm{e1}+\mathrm{A} \mathrm{e2} \text {. }
$$

In terms of our nomenclature, the parametric equation for a line along the normal vector is:

$$
X N=-P y(i)-[P y(i-1)-P y(i)]^{*}
$$

To find the point along the normal vector where it intersects with the baseline spline you must solve to equations simultaneously for the two unknowns (s and $t$ ). The parametric equations for the baseline WF spline are:

$$
\begin{aligned}
& X=A x^{*} s^{*} s^{*} s+B x^{*} s^{*} s+C x^{*} s+D x \\
& Y=A y^{*} s^{*} s^{*} s+B y^{*} s^{*} s+C y * s+D y
\end{aligned}
$$

After the line along the normal vector is defined, you can intersect it with the baseline spline. If the closest point from the spline to the evaluation point is greater than the specified tolerance, the interval must be subdivided. you subdivide in the center of each interval.

Once an evaluation point is found outside the tolerance band, you do not need to evaluate the remainder of the interval since it will be subdivided anyway.

Let us assume that regardless of how refined the temporary spline is, comparing 2000 evenly spaced points along the spline's arc length renders a sufficient comparison (2000 plus a little room equals MAXEVALS). Determine how many points will be evaluated within each through point interval (round off). you define the parameters of the tangent line as:

$$
\mathrm{T}=\mathrm{A} 1 \mathrm{e} 1+\mathrm{A} 2 \mathrm{e} 2
$$

where

$$
\begin{aligned}
A 1 & =X 0+A L C * t \\
& =P x(i)+[P x(i-1)-P x(i)]^{*} t
\end{aligned}
$$

and

$$
\begin{aligned}
\mathrm{A} 2 & =\mathrm{Y} 0+\mathrm{BLC}{ }^{*} \mathrm{t} \\
& =\mathrm{Py}(\mathrm{i})+[\mathrm{Py}(\mathrm{i}-1)-\mathrm{Py}(\mathrm{i})]^{*} \mathrm{t}
\end{aligned}
$$

The equation of the normal is defined as follows:

$$
\mathrm{N}=(-\mathrm{BLC}+\mathrm{X} 0) \mathrm{e} 1+(\mathrm{ALC}+\mathrm{Y} 0) \mathrm{e} 2
$$

The normal line is the line equation passed to the error function.

The number of points evaluated will be such that DS $>=$ tolerance. This way, as the subdivided intervals get smaller, the number of times they are evaluated decreases. you determine how many times to evaluate within each spline interval by measuring the total parametric length of the spline 
and computing the Delta $L$ interval length. Evaluate no more than 200 times within any through point interval.

Instead of simply looping through this evaluation process NTPTS times, you will perform a quality check at the end of each evaluation to determine whether or not to continue.

If the temporary spline has converged to the baseline spline, you are done. Convert the virtual spline definition into the new temporary spline definition.

3. SUBDIVIDE (T_SPL, I, DS, BL_SPL, NBLPTS, SPLTYP, V_SPL,NVPTS)

\section{INPUT PARAMTTERS:}

T_SPL - Temporary spline array

I - Interval to be subdivided

DS - Delta parametric arc length to use to search the baseline spline

BL_SPL - Baseline spline array

NBLPTS - Number of baseline spline defining points

SPLTYP - Type of baseline spline

OUIPUT PARAMITERS:

V_SPL - Newly defined temporary spline definition array

NVPTS - Number of defining points in new temporary spline

This subroutine finds the intervals where the temporary spline was not equivalent to the baseline spline and subdivides the temporary spline. The newly subdivided temporary spline definition will be stored in the V_SPL arrays.
The temporary spline is subdivided near its midpoint. Start the subdivison process by computing the center of temporary spline's interval that is to be subdivided. Then find the closest point along the baseline spline to the computed subdivision point on the temporary spline.

The computed point on the baseline spline becomes the new through point for the temporary spline.

Shuffle the V_SPL arrays to make room for this new through point.

Once the temporary spline arrays are shuffled to make room for insertion data, find the midpoint of the temporary spline's Ith interval. Then find the closest point on the baseline spline to this temporary spline point.

The call to function ERROR finds the nearest point along the baseline spline to the midpoint of the temporary spline's Ith interval. Since you are trying to converge to the baseline spline, you use its computed point to define the location of the new through point on the temporary spline. It does not particularly matter that you get the exact midpoint.

Shuffle the virtual spline arrays to make room for a new through point. Compute the new coord length for the Ith interval. Then add the coord length and coordinates of the new through point to the V_SPL array.

4. GET_SPLPT(SPLINE, IKNOT, S, $\mathbf{X}, \mathbf{Y}$

INPUT PARAMETERS:

SPLINE - Spline from which parametric definition is specified 
IKNOT - Knot point interval that computed point lies in.

S - Parametric distance from start of interval that point lies

\section{OUTPUT PARAMTTERS:}

X -

$\mathrm{X}$-coordinate of computed point

$\mathbf{Y}$ Y-coordinate of computed point

This subroutine computes the coordinates of a point in the IKNOTth interval for the input spline contained in the SPLINE array.

The spline is currently assumed to be a cubic spline. The desired coordinate location is specified in terms of the parametric arc length $S$.

Hence, for a given spline representation the coordinates of a point in the ith interval a distance $S$ from the intervals start location is:

$$
\begin{aligned}
X= & \text { SPLINE }(2, I K N O T)+ \\
& \text { S*SPLINE(3,IKNOT) }+ \\
& \text { S*S*SPLINE(4,IKNOT) + } \\
& \text { S*S*S*SPLINE }(5, I K N O T)
\end{aligned}
$$

$$
\begin{aligned}
& Y=\operatorname{SPLINE}(6, \mathrm{IKNOT})+ \\
& \text { S*SPLINE(7,IKNOT) + } \\
& \text { S*S*SPLINE(8,IKNOT) + } \\
& \text { S*S*S*SPLINE(9,IKNOT }
\end{aligned}
$$

5. ERROR (ALC, BLC, XO, YO, SPLINE, NPTS, SPLTYP, OLDS, BLX, BLY)

\section{INPUT PARAMETERS:}
ALC - Coefficient of normal
BLC - Coefficient of normal

XO - X-coordinate of computed point

Y0 - Y-coordinate of computed point

SPLINE - Baseline spline

NPTS - Number of baseline points

SPLTYP - Type of baseline spline

OLDS - Last value of parameter $\mathbf{S}$

\section{OUTPUT PARAMETERS:}

BLX - X-coordinate of the nearest point on the SPLINE

BLY-Y-coordinate of the nearest point on the SPLINE

This function computes the minimum distance from the input point $(\mathrm{X} 0, \mathrm{YO})$ to the input spline (SPLINE).

The algorithm begins by using the input equation for a line passing through the point $\mathrm{XO}, \mathrm{YO}$ (this line represents a normal to another curve). The algorithm then intersects that line with the baseline spline.

If the baseline spline is a cubic (e.g., WF), the line and spline are set equal to each other to compute the intersection. This involves solving two equations simultaneously to find the cubic roots.

The line that intersects the spline is of the form:

$$
\text { LINE }=\mathrm{A} \mathrm{e} 1+\mathrm{B} \text { e2 }
$$

where

$$
\begin{array}{ll}
A=A L C * T+X 0, & A L C \Rightarrow X \cdot X 0 \\
B=B L C * T+Y 0, & B L C \Rightarrow Y-Y 0
\end{array}
$$

The form of the cubic spline is:

$X=A X * S * S * S+B X * S * S+C X * S+D X$ $Y=A Y^{*} S^{*} S^{*} S+B Y^{*} S * S+C Y * S+D Y$ 
At the intersection point:

$$
\begin{aligned}
A L C * T+X 0 & =A X * S * S * S+B X * S^{*} S \\
& +C X * S+D X \\
B L C * T+Y 0 & =A Y * S * S * S+B Y * S * S \\
& +C Y * S+D Y
\end{aligned}
$$

These two equations are solved simultaneously. They can first be solved for $\mathrm{S}$ by solving an equation of the form:

$$
A * S * S * S+B * S * S+C * S+D=0
$$

Where

$$
\begin{aligned}
& \mathrm{A}=[(\mathrm{BLC} / \mathrm{ALC}) * \mathrm{AX}-\mathrm{AY}] \\
& \mathrm{B}=\left[(\mathrm{BLC} / \mathrm{ALC})^{*} \mathrm{BX}-\mathrm{BY}\right] \\
& \mathrm{C}=\left[(\mathrm{BLC} / \mathrm{ALC})^{*} \mathrm{CX}-\mathrm{CY}\right] \\
& \left.\mathrm{D}=\left[(\mathrm{BLC} / \mathrm{ALC})^{*}(\mathrm{DX}-\mathrm{X} 0)+\mathrm{YO}-\mathrm{DY}\right)\right]
\end{aligned}
$$

We don't need to know $T$ for any reason, but if you did it would be:

$$
\begin{aligned}
T= & (A X / A L C) * S * S * S+ \\
& (B X / A L C) * S * S+(C X / A L C) * S+ \\
& {[(D X-X 0) / A L C] }
\end{aligned}
$$

Once the intersection point is found, a sweep is made in the neighborhood of the intersection point to determine if there is another point on the baseline spline closer to the in put coordinates.

Closeness is defined as the closest linear distance. you do this because the closest distance between two splines may not be normal to one spline's curve. The square of the minimum distance is returned as the error at the point.

Determine the interval on the baseline spline where the intersection is most likely to occur. The equation of the line represents a normal to some other spline at a particular point.
Define the four coefficients used to solve for the intersection point's spline parameter S. Use the Horner's method of synthetic division to find a root for this cubic.

Horner's method of synthetic division may not find a distance less than tolerance.

You now know the normal intersection of the temporary spline with the baseline spline. Geometrically speaking, the normal intersection may not provide the nearest point on the baseline spline to the temporary spline's XY location. To ensure that you find the nearest point, a sweep is made in the neighborhood of the intersection point to determine if any neighbor points are closer.

Search in both directions (+S and $-S)$. The If-logic used is necessary since the cubic root solver can be unstable depending on the condition of the coefficients.

Do the high side of life. Provide logic for unstable cubic roots. The $X$-coordinate was trapped in the SPLINE interval INT between the parameters S_LOW and S_HI.

Evaluate SPLINE from S_LOW to S_HI at NSLES evenly spaced points. Find the closest point on SPLINE to the $X Y$-coordinate by computing the linear distance between each NSLES evaluation point on SPLINE and the $\mathrm{XY}$-coordinate. Keep track of the value of $\mathrm{S}$ used in this iteration.

6. HORNERS (A, OWDS, S) INPUT PARAMETERS:

A - Spline coefficient array

OLDS - Last used value of S. Initial value for the cubic solver 


\section{OUTPUT PARAMETHRS:}

S - Root of the cubic equation or parameter of spline where root exists

This subroutine uses Horner's method of synthetic division. It computes the roots of a cubic equation given an initial value for the cubic's parameter (OLDS).

The root will be returned in the variable S. Horner's method can be used to solve for the roots of nth ordered polynomials but it is coded here for strictly cubics. Horner's method is a variation of Newton's method.

The cubic equation to be solved is of the form:

$$
\begin{aligned}
& P(S)= A(1) * S * * 3+A(2) * S * * 2+ \\
& A(3) * S+A(4)
\end{aligned}
$$

The derivative of the cubic equation is:

$$
\begin{aligned}
& \mathrm{DP}(\mathrm{S})= 3^{*} \mathrm{~A}(1) * \mathrm{~S}^{* * 2}+2 * A(2) * S+ \\
& \mathrm{A}(3)
\end{aligned}
$$

For an initial parameter (OLDS), the root of the cubic is found by

$$
S=\text { OLDS - [P(OLDS) / DP(OLDS })]
$$

The first time you compute a root, it may not render the best value. you therefore iterate potentially ten times.

7. GET_INTERVAL (AL, BL, XLO, YLO, SPLINE, NPTS, SPLTYP, INT)

\section{INPUT PARAMETERS:}
AL - $\quad X$-coefficient of input line
BL - Y-coefficient of input line
XLO - X-coefficient of input line
YLO - Y-coefficient of input line
SPLINE - Coefficient array of input spline

NPTS - Number of defining points in the input spline

SPLTYP - Input spline's type OUTPUT PARAMTETERS:

INT - Spline interval in which the linespline intersection occurs

This subroutine computes the spline interval in which the input line intersects with the input spline. The intersection interval can be computed by generating $\mathbf{N}$ lines connecting the $\mathbf{N}$ through point intervals of the SPLINE and performing a line-line analytical intersection.

The equations for intersecting a line with a line can be generated a number of different ways. The method used here is to write a set of parametric equations for both lines and set them equal to each other.

The set of equations for the input line are:

$$
\begin{aligned}
& X=A L * t+X L O \\
& Y=B L * t+Y L O
\end{aligned}
$$

The set of equations for the line connecting two through points are:

$$
\begin{aligned}
& \mathrm{X}=\mathrm{AS}^{*} \mathrm{~s}+\mathrm{XSO} \\
& \mathrm{Y}=\mathrm{BS}^{*} \mathrm{~s}+\mathrm{YSO}
\end{aligned}
$$

To determine if two lines intersect, set them equal to each other:

$$
\begin{aligned}
& X \Rightarrow A L * t+X L 0=A S^{*} s+X S 0 \\
& Y \Rightarrow B L * t+Y L O=B S^{*}+Y S 0
\end{aligned}
$$

From these simultaneous equations, you get the following equations:

$$
\begin{aligned}
t= & (\mathrm{AS} / \mathrm{AL}) * \mathrm{~s}+(1 / \mathrm{AL}) *(\mathrm{XSO}-\mathrm{XLO}) \\
\mathrm{s}= & {[\mathrm{BL} / \mathrm{DENOM}]^{*}(\mathrm{XSO}-\mathrm{XLO})+} \\
& {\left[\mathrm{AL} / \mathrm{DENOM}{ }^{*}(\mathrm{YLO}-\mathrm{YSO})\right.}
\end{aligned}
$$

Where 
$D E N O M=A L * B S-A S * B L$

The parameter $\mathbf{S}$, used to define the line through the two spline points, is equal to zero at the start of the interval and one at the end of the interval.

Therefore, you know whether or not the intersection was successful by the value of $\mathbf{S}$.

For intersection, $0<=S<=1$.

It is possible that the spline is not monotonic. Hence, the input line may intersect the spline at more than one location. you keep track of multiple intersections. The intersection resulting in the smallest absolute value of $t$ is the one you want (smallest $t$ means closest to the start point of the input line).

8. ANG_INC (P,LEN, ANG,KNOT)

INPUT PARAMETERS:

$P$ - Input spline array of coefficients

LEN - Number of defining points OUTPUT PARAMETERS:

ANG - Value of largest computed angular increment

KNOT - Interval where largest angular increment was computed

This subroutine computes the maximum angular increment for the input spline ( $P$ ). The angular increment is taken as the change in siope at each knot point and is computed using direction vectors through the $(i-1, i)$ and $(i, i+1)$ knot point intervals. Compute the two vectors through the Ith knot point and the angle between them. Angular equation is from law of cosines pg. 19, Davis and Snider.
9. TRAP_COORD (SPLINE, NPTB, TYPE, INT, X, 8_LOW, 8_HIGH) INPUT PARAMETERS:

SPLINE - Input spline

NPTS - Number of defining points in input spline

TYPE - Input spline's type

INT - Initial spline search interval

$X$ - X-coordinate of point that is being trapped

OUTPUT PARAMETERS:

INT - Interval where point $X$ was trapped

S_LOW - Spline parameter that yields a spline value just smaller than X

S_HIGH - Spline parameter that yields a spline value just larger than $\mathrm{X}$

This subroutine finds the values of the parameters S_LOW and S_HIGH that trap the $\mathrm{X}$-coordinate $\mathrm{X}$ in the spline SPLINE.

The parameter INT is the spline interval where the search will begin. When done, it is the spline interval where $\mathrm{X}$ was trapped.

Use the $x$-coordinate to determine the neighborhood of $x y$ points with respect to the spline. Use that computed position and points on both sides within a neighborhood to determine tangent vectors. Determine if a shorter point lies along the tangent vectors.

Determine what spline type you are working with. Then determine if the $\mathrm{XY}$ point is within the current evaluation interval. If it is not, it must 
be in the next interval (because of our assumption on monotonicity).

If the baseline spline type is WF, then the spline being evaluated in this routine is a CAE spline.

To find the closest point to the XY coordinate, trap the $X$ coordinate the IKNOTth interval. you can do this because of our assumption on monotonicity. If the $\mathrm{X}$ coordinate is not in the IKNOTth interval, search the IKNOTth+1st interval. Once the $X$ coordinate is trapped, find the closest point within a neighborhood of the trapped point to the XY coordinate.

10. FIND_LOW (SPLINE, NPTS, TYPE, X, INT, g)

INPUT PARAMETERS:

SPLINE - Input spline

NPTS - Number of defining points in input spline

TYPE - Input spline's type

X - X-coordinate of point to try to find on spline

\section{OUTPUT PARAMETERS:}

INT - Spline interval where $x$ coordinate was found

S - Spline parameter for largest $x$ coordinate on spline that is less than input value of $X$

This function finds the largest $X$-coordinate on SPLINE that is less than the input value $X$.

Determine if a normal from the spline is the closest point between the spline and the input point. Use accuracy to specify points on both sides of the normal to investigate.

Determine what spline type is being evaluated. If the logical TYPE is true, the baseline spline is WF $s 0$ the spline being evaluated is a CAE opline. Otherwise, the baseline spline is a CAE spline and the spline being evaluated is WF. Step along the spline's current interval until the X-coordinate of the spline evaluation point is less than $X$.

If you don't find a opline $X$-coordinate less than $X$ in the INTth interval, try to find the epline coordinate in the next interval. Find the largest value of SPLX that is less than S using delta S's of DS.

Begin with the input value for the spline interval (INT), and the parameter within that interval (S). Output the interval found and its parameter (INT and S). After the first value of SPLX is found that is less than the value of $X$, continue evaluating until the largest value of SPLX less than $X$ is found.

\section{FIND_HIGH (SPLINE, NPTS, TYPE, X, INT, S)}

INPUT PARAMITTERS:

SPLINE - Input spline

NPTS - Number of defining points in input spline

TYPE - Input spline's type

X - $\quad$ X-coordinate of point to try to find on spline

\section{OUTPUT PARAMETERS:}

INT - Spline interval where $x$ coordinate was found

S - Spline parameter for smallest $x$ coordinate on spline that is less than input value of $X$

This function finds the smallest $x$-coordinate on SPLINE that is greater than the input value $X$. 
When the function FIND_LOW is called prior to this function call, the input parameter $\mathbf{S}$ plus DS should yield the $x$-coordinate you're looking for. In other words, this function should converge in one step if called immediately after a call to function FIND_LOW.

Determine if the normal is the closest point between splines. Use acc to specify points on both sides of the normal to investigate. Determine what spline type is being evaluated to find $\mathrm{X}$-coordinates.

If the logical TYPE is true, the baseline spline is WF 80 the spline being evaluated is a CAE spline. Otherwise, the baseline spline is a CAE spline and the spline being evaluated is WF.

Step along the spline's current interval until the X-coordinate of the spline evaluation point is greater than $X$. If you don't find a spline $\mathrm{X}$-coordinate greater than $\mathrm{X}$ in the INTth interval, try to find the spline coordinate in the next interval.

Find the smallest value of SPLX that is greater than S using delta S's of DS. Begin with the input value for the spline interval (INT) and the parameter within that interval (S). Output the interval found and the its parameter.

If this function is call immediately following a call to function FIND_LOW, variable $S$ plus $D S$, should yield the $x$-coordinate you're after. After the first value of SPLX is found that is greater than the value of $X$, continue evaluating until the smallest value of SPLX greater than $X$ is found.

Determine the maximum value of $S$ for this interval.
12. GET THMPGPL (SPLNE, NPTB̄, SPLTYP, TMP_EPL, NTPTS)

INPUT PARAMETERS:

SPLINE - Input spline.

NPTS - Number of defining points in input spline.

SPLTYP - Input spline's type

OUTPUT PARAMETERS:

TMP_SPL - Array containing the coefficients of the temporary opline

NTPTS - Number of defining points in temporary spline

This subroutine gets the temporary spline definition. If the temporary spline is a CAE spline (baseline spline is a WF), spawn a process to generate a CAE spline using the through points in the SPLINE array.

If the baseline spline is a CAE spline, generate a WF spline. When NTPTS $=0$, get through points from baseline spline. Otherwise read the TMP_SPL array to generate WF spline.

\section{CUBIC (COEF, NROOTS, RROOT)}

\section{INPUT PARAMETERS:}

COEF -Input spline array of coefficients OUTPUT PARAMETERS:

NROOTS - Number of real distinct roots found

RROOTS - Real values of roots found

This subroutine computes the roots to a cubic equation defined by the cubic coefficients COEF. RROOT contains the real values of the roots. NROOTS 
is the number of real distinct roots found.

The source for the analytical equations solved in this algorithm is CRC Mathematical handbook.

Normalize cubic coefficients with respect to the $Y^{* *} 3$ coefficient. Solve the equation:

$$
Y * * 3+P P * Y * * 2+Q Q * Y+R R=0
$$

Determine the number of roots and their real values. Determine the number of roots you have. Check to see how many real distinct roots their are. If

$$
\frac{\mathrm{BB}^{* * 2} \mathrm{AA}^{* * 3}}{4+27}>0,1 \text { REAL ROOT }
$$

The problem is that roots to a cubic equation are heavily dependent on the cubics coefficients. If the coefficients are off due to roundoff or something, you could be in big trouble.

Assume that the root with the smallest imaginary part is the one real root.

$$
\begin{array}{ll}
\text { RMIN } & =\text { ROOT(1) }- \text { PP/3.D0 } \\
\text { RMIN2 } & =\text { ROOT(2) - PP/3.D0 } \\
\text { RMIN3 } & =\text { ROOT(3) }- \text { PP/3.D0 } \\
\text { RROOT(1) } & =\text { RMIN }
\end{array}
$$

\section{SPAWN_PROC (TMP_SPL, NTPTS, AIN, AOUT)}

INPUT PARAMETERS:

TMP_SPL - Input spline's coefficient array

NTPTS - Number of defining points in input spline

\section{OUTPUT PARAMETERS:}

AIN - Start angle for CAE system spline, if end angles are used
AOUT - End angle for CAE system spline, if end angles are used

This subroutine spawns a process that does three things. First, it writes the baseline spline into a CAE ready IGES file. Next, it executes the CAE system, which reads the spline in, generates a CAE spline, and outputs CAE-spline in an IGES format.

Finally, the process reads the CAE spline into the $\mathrm{SE}$ module and quits.

\section{APT Wilson-Fowler Spline Module}

The APT spline module contains subroutines from the CAMI-APT family of software tools.

The CAMI-APT software tools have been around for over twenty years and are considered a defacto standard set of machining software tools. For this reasons, you did not alter the subroutines when you incorporated them into our IS family of subroutines.

By using unaltered CAMI-APT subroutines, users can be sure that when they use the IS tool, they are comparing their CAE system spline algorithm against not only a known standard, but against the spline algorithm that may be used to generate the numerical controlled (NC) instructions for the machine that cuts the part.

Many of the unnecessary lines of code where commented out. Those lines are clearly marked. Some arrays were also increased.

\section{APTWF (WFSPL, NBLPTS)} INPUT PARAMETERS:

WFSPL - Spline array that contains the defining point data 
NBLPTS - Number of defining points OUTPUT PARAMETERS:

WFSPL - Spline array containing the defining point data and the full set of coefficients

This subroutine controls the generation of the baseline APT Wilson-Fowler spline. The main features of this subroutine are to put the IS standard data format into an APT format and to call two main APT routines (APT088 and APT089).

The reason that you convert to the APT data format is because you decided to not make any changes to the original APT program, thus preserving our purest baseline assumptions.

Subroutine APT088 takes the initial through point data, computes slope segment angles and segment lengths and load the TAB array in the proper format. After APT088 is done, routine APT089 can be called to compute the WF cubic coefficients. The spline fitting method is:

1. Approximate the slopes at each interior point by assigning each point the slope of a circle which passes through that point and the adjacent point on each side (3 point circle).

2. At each interior point calculate the difference in curvature between the cubic equation on one side of the point and the cubic equation on the other side of a point (i.e., delta curvature) in terms of the exterior angles.

The final form of the data array is:

tab(1) - Record number of external canonical form

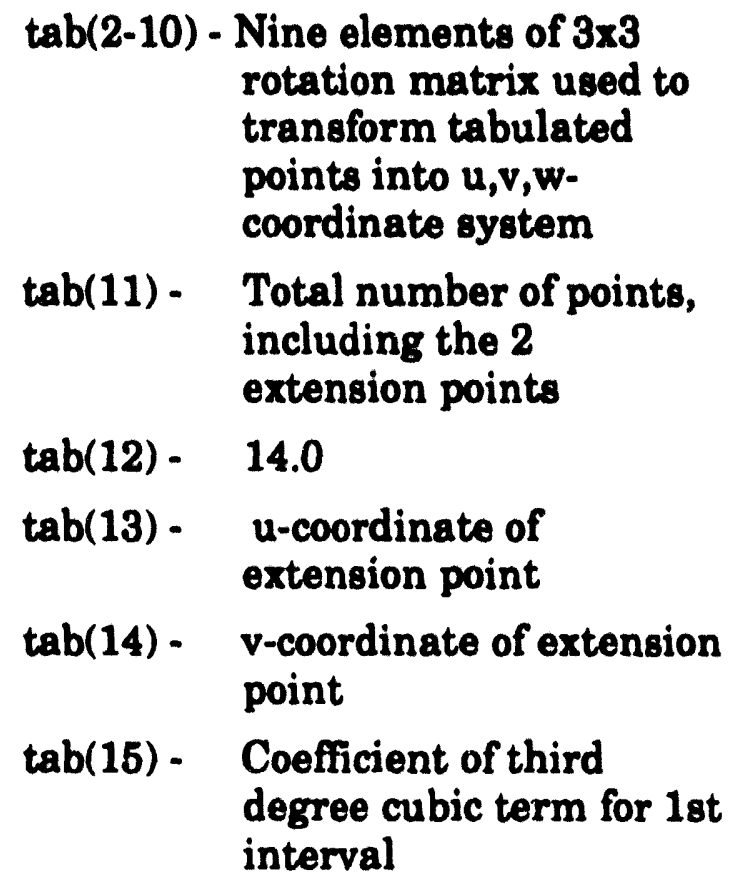

tab(11) - Total number of points, including the 2 extension points

$\operatorname{tab}(12)-\quad 14.0$

tab(13) - u-coordinate of extension point

tab(14) - v-coordinate of extension point

$\operatorname{tab}(15)$ - Coefficient of third degree cubic term for 1st interval

tab(16) - Coefficient of second degree cubic term for 1st interval

tab(17) - Length of 1st interval

tab(18) - Maximum value of cubic in 1st interval

tab(19) - Minimum value of cubic in 1st interval

$$
\begin{aligned}
& \operatorname{tab}(13+m)-u \text {-coordinate of } m \text { th } \\
& \text { point }(m=1, . ., n \text { and } n= \\
& \text { \# of through pts) } \\
& \operatorname{tab}(14+m) \cdot v \text {-coordinate of } m \text { th } \\
& \text { point } \\
& \operatorname{tab}(15+m) \text { - Coefficient of third } \\
& \text { degree cubic term for } \\
& m \text { th interval } \\
& \text { tab }(16+m) \text { - Coefficient of second } \\
& \text { degree cubic term for } \\
& \text { mth interval } \\
& \operatorname{tab}(17+m) \text { - Length of } m \text { th interval }
\end{aligned}
$$




$$
\begin{aligned}
& \operatorname{tab}(18+\mathrm{m})-\begin{array}{l}
\text { Maximum value of } \\
\text { cubic in mth interval. }
\end{array} \\
& \begin{array}{ll}
\operatorname{tab}(19+\mathrm{m})- & \begin{array}{l}
\text { Minimum value of } \\
\text { cubic in mth interval. }
\end{array} \\
: & : \\
: & : \\
\operatorname{tab}(13+n)- & \begin{array}{l}
\text { u-coordinate of last } \\
\text { extension point }
\end{array} \\
\operatorname{tab}(14+n)- & \begin{array}{l}
\text { v-coordinate of last } \\
\text { extension point }
\end{array}
\end{array}
\end{aligned}
$$

After the APT WF spline is computed, it is defined as a series of local $(u, v)$ coordinate systems. Each local coordinate system is defined by the cubic:

$$
v(u)=c 1^{*} u^{* *} 3+c 2^{*} u^{* *} 2+c 3^{*} u+c 4
$$

By applying boundary conditions on the local interval $\mathrm{v}(0)=0, \mathrm{v}(\mathrm{L})=0$

$$
\mathrm{v}^{\prime}(0)=\mathrm{TA}, \mathrm{v}^{\prime}(\mathrm{L})=\mathrm{TB}
$$

The local cubic reduces to

$$
v(u)=c 1^{*} u^{* *} 3+c 2 * u^{* *} 2+c 3^{*} u,
$$

where $c 3=$ TA since $v(L)=0$

we get that

$$
c 1^{*} u^{* *} 3+c 2^{*} u^{* *} 2+c 3^{*} u=0
$$

Which implies:

$$
c 3=-c 1 * u * * 2-c 2 * u
$$

After the series of local cubics is defined, they will be transformed into global representation this will involve translation, rotation, and a reparameterization. The new parameterization is with respect to the spline's cumulative coord length. ${ }^{1}$

Determine if end angles are to be specified or computed. KK and LL are end angle flags. When they $=1$, end angles exist and $=0$ no end angles exist. The KK flag is for the entry and the LL flag for exit of spline. When end angles (slopes) are given, they should be in radians. APT defines the slope to be the tangent of the start or end angle.

What you want to give the APT routines is the Tangent of the end angles. Define the necessary APT parameters. $\mathrm{MM}$ is a coordinate definer, $=3 \Rightarrow X Y$ coordinate data.

$\mathrm{JJ}$ is a T\&R flag, $=0 \Rightarrow$ no data translation or rotation. Load the through points into the APT array and compute the initial set of local intervals.

Compute the series of local cubics defined over each through point interval. Convert the series of local cubics into a global spline definition with a cumulative chord length parameterization.

Begin by computing the linear term for each of the local cubic equations. Always compute the end angles after computing APT WF coefficients. Return angles in radians.

2. APT088 (A, MM, NN, KK, LL, JJ, TAB, ANGIN, ANGOUT)

\section{INPUT PARAMETERS:}

A - Array of defining point data

MM - Flag for demensionality

NN - Number of defining points

KK - Entry angle flag; =1, entry angle exist and $=0$, no entry angle

LL - Exit angle flag; =1, exit angle exists and $=0$, no exit angle

1 R. M.,Dolin, "The Wilson-Fowler Spline in a Global IGES Coordinate Frame," Los Alamos National Laboratory Report Number LA-11024-MS, (September, 1987). 
JJ - T\&R flag; =0, $\Rightarrow$ no data translation or rotation

OUTPUT PARAMETERS:

TAB -

Array containing canonical form of APT-WF spline

ANGIN - Computed entry angle, when not supplied

ANGOUT - Computed exit angle, when not supplied

This subroutine reads defining point data $(\mathrm{XY})$ and end angles, and puts the data into a form that can be used by APT089 to generate an APT Wilson-Fowler spline.

Comment lines with an rmd or cd moniker were added by Ron Dolin and comment lines with an RJG or CG moniker were added by Ralph Gladfelter.

The first section defines local functions that are used by this subroutine. They represent equations from the original Fowler and Wilson report.

There are several functions locally defined in this subroutine. The first function returns the smaller of either the input number or 1e-9. The reason for this function is to insure that you never get into a numerical divide by zero situation.

The second function computes the slope, tangent, and curvature of the input variables. This function assumes knowledge of the delta X's and Y's for the interval represented by $\mathrm{Z1}$ and $\mathrm{Z2}$. The equation used by this function is equivalent to equation 9 of the Y-1400 "Cubic Spline, In A Curve Fitting Routine Report By Fowler and Wilson."
The next function computes the difference in curvature at a given through point. There are two measures of curvature at each through point. The interval to the left and the interval to the right both have curvatures that need to be compared so that they can be checked for convergence. It calls function CRVA from above. It uses an equation that is equivalent to equation 18 of the Y-1400 "Cubic Spline, A Curve Fitting Routine Report By Fowler And Wilson."

A majority of the subroutine is not necessary for the particular application of the APT WF-spline representation that you have and is therefore commented out. The only functionality that we really need is the data manipulation and initial data calculation necessary to run the WF algorithm. We decided not to move lines around, so read carefully to see what has been commented out and what has not.

The spline data should now have been transformed into the proper structure to be used by the subroutine that actually computes the cubic coefficients of the spline (APT089). In any event, our work here is done. We have specified end conditions based on a circle through the first and last three points of the spline. If the end angles where not initially specified, use these computed end angles. 
3. APT089 (TAB, ANGIN, ANGOUT)

INPUT PARAMIETERS:

TAB -

Array of defining point data

ANGIN - Entry slope

ANGOUT - Exit slope

OUTPUT PARAMETERS:

TAB - Array of defining point data and WF spline coefficients

This subroutine generates the APT version of the WF spline. The first section defines local functions that are used by this subroutine. They are the same functions defined in the previous section.

A majority of this subroutine is not necessary for the particular application of the APT WF-spline representation that you have and is therefore commented out.

The only functionality that you really need is the data manipulation and initial data calculation necessary to run the WF algorithm.

We decided not to move lines around, so read carefully to see what has been commented out and what has not. The next hundred or so lines have executable lines intermixed.

Find the maximum value of the delta curvature at each of the (2nd) to $\mathrm{n}-1$ through point intervals. If all the interval delta curvatures where less than 1e-3, you can skip the curvature minimization stuff. However, if even one through point interval did not have continuous curvature, you must minimize it. If several intervals had discontinuous curvatures, minimize the interval that was the most out of tolerance.

Changes to the slope and curvature at the ith interval impacts the computed slope and curvature at the $i-1$ st and $i+1$ st intervals, which in turn impacts their $j-1$ st and $j+1$ st intervals. In other words, changing the slope and curvature in one interval can impact all others.

The first computed "go to" directs work depending on whether the start angle has been specified (e.g., $610 \Rightarrow$ no start angle). The second computed "go to" directs flow depending on whether the end angle has been specified.

\section{APTO40 (RESULT, VC)}

INPUT PARAMETERS:

RESULT - Array containing the canonical form of the resulting vector

\section{OUTPUT PARAMETERS:}

VC - Array containing the canonical form of the input vector

This subroutine generates the canonical form of a vector defined as the normalization of a given vector by the following APT statement

RESULT $=$ VECTOR/NIT, VC

For our use, this subroutine normalizes a vector by calling the VNORM subroutine.

5. VNORM (VA, V3) INPUT PARAMETERS:

VA - Input vector array OUTPUT PARAMETERS:

V3 - Output normalized vector array 
This subroutine normalizes a vector. you can't really say much more than that.

\section{GET_ANG (WFSPL, NPTS, ANGIN, ANGOUT) \\ INPUT PARAMITERS:}

WFSPL - Array of spline data.

NPTS - Number of spline points.

OUTPUT PARAMETERS:

ANGIN - Computed entry angle

ANGOUT - Computed exit angle

This subroutine computes the entry and exit angles of the spline using the spline's cubic coefficients. If the coefficients do not exist or if the current form of parameterization is bad, a new set of angles is computed using the three point circular method used by Fowler and Wilson and presented in function DANGLE.

Using limit theory, the entry slope is the limit, as the parameter " $\mathrm{s}$ " approaches zero of the derivatives of $\mathrm{Y}(\mathbf{s}) / \mathrm{X}(\mathrm{s})$. Given the two governing cubic equations:

$$
\begin{aligned}
& \mathrm{X}(\mathrm{s})=\mathrm{AXs}^{3}+\mathrm{BXs}^{2}+\mathrm{CXs}+\mathrm{DX} \\
& \mathrm{DX}(\mathrm{s})=3 \mathrm{AXs}^{2}+2 \mathrm{BXs}+\mathrm{CX}
\end{aligned}
$$

and

$$
\begin{aligned}
& Y(s)=A Y s^{3}+\mathrm{BYs}^{2}+\mathrm{CYs}+\mathrm{DY} \\
& \mathrm{DY}(\mathrm{s})=3 \mathrm{AYs}^{2}+2 \mathrm{BY}+\mathrm{CY}
\end{aligned}
$$

the slope is:

$$
A=\operatorname{Tan}^{-1}\left[\lim _{s->0}(D Y(s) / D X(s)]\right.
$$

\section{INPUT/OUTPUT MODULE}

This module controls the ASCII and IGES input/output for the Interchangeable Spline program (IS).
The IS program will read full IGES 112 and 126 spline entities and write a 112 spline entity containing only the spline through-points and end angle information. For now, only the IGES 112 entity is used.

Subroutines in this module include:
GET_WF,
PUT_WF,
GET_IGES, PUT_IGES, IGES_IN, READ_IGES, READ_DE, P6RRAF, READ_WF, READ_112, DFREFLD, IGES_OUT, P6WINI

\section{GET_WF(WF_SPL, NWFPTS, ANGIN, ANGOUT)}

\section{INPUT PARAMETERS:}

None

\section{OUTPUT PARAMETERS:}

WF_SPL - Array containing APT-WF spline

NWFPTS - Number of spline points ANGIN - Entry angle for spline ANGOUT - Exit angle for spline

This subroutine gets a spline from the current working directory. It assumes that the spline file is named WF2SE.DAT and is in an ASCII XY data format. The first line of the ASCII file specifies the number of XY-tuples that makeup the spline's set of defining points. 
The next NPTS lines are the XY-tuples. If there is an NPTS 1 line in the file, it contains the splines end angles. The spline's start angle will reside in the $X$-field and the exit angle will reside in the Y-field.

If the end angles do not exist, the angle flag (IAFLG) is set to 0 , otherwise its set to 1 . It is assumed that the end angles are in degrees.

Open the file in the current working directory called WF2SE.DAT Read the first line of the file and determine the number of tuples. Read the next NBLPTS lines of the file as defining points. Read the last line of the data file, if it exists, as the spline's end angle data. If the end angles exist, they are in degrees, so convert them to radians.

If the end angles do not exist, the WF algorithm will compute default end angles. Generate the full WF spline definition.

2. PUT_WF (WF_SPL, NWFPTS, ANGIN, ANGOUT)

INPUT PARAMETERS:

WF_SPL - Array containing spline definition

NWFPTS - Number of spline points

OUTPUT PARAMETERS:

ANGIN - Spline's entry angle

ANGOUT - Spline's exit angle

This algorithm puts the current WF spline into the user's current working directory. The spline is stored in file SE2WF.DAT and is in an ASCII XY data format.

The first line of the ASCII file specifies the number of XY-tuples that make up the WF spline's set of through-points. The next NWFPTS lines are the XY-coordinates of the spline's defining points. The NPTS+1 line in the file contains the spline's end angles.

The spline's start angle resides in the X-field and the exit angle resides in the Y-field. It is assumed that the end angles are in degrees.

Open the file in the current working directory called SE2WF.DAT. Write the first line of the file (number of defining points). Write the next NWFPTS lines of the file (the defining points). Write the last line of the data file (the end angles in degrees). 3. GET_IGES (IG_SPL, NSPTS,
SPL_TYP)

INPUT PARAMETERS:

None

OUTPUT PARAMETERS:

IG_SPL - Array containing the IGES spline

NSPTS - Number of spline points

SPL_TYP - Type of spline found

This subroutine gets an IGES formatted file. IGES files contains five sections that each have a unique structure. The first section is called the GLOBAL section and provides hardware/machine dependent information.

The second section is called the START section and contains information about the data, such as plot titles, etc.

The third section is called the DIRECTORY ENTRY section and contains information about each of the 
geometry entities in the file, such as type of entity, label, how it should be displayed, level it lives on, etc.

The DE section also contains a pointer to the Parameter Data (PD) section where the coordinate data for the geometry lives. The final section, called the TERMINATOR section, contains an accounting of how large each of the other sections were.

Each section is disseminated by different letters in the 72nd column of each line. The global section has $\mathbf{a} \mathbf{G}$ in column 72, the start section an $S$, the directory entry section $a D$, the parameter data section a $P$ and the terminator section a $T$. The actual coordinate data is stored in the PARAMETER DATA section.

For splines, the format of the 112 and 126 entities can vary. Even the interpretation of the 112 entity can vary depending on the spline type being read.

This subroutine assumes that the IGES input file is called CAE2IS.IGS and contains a single spline entity. The type of entity (112 or 126) depends on the CAE system.

Open the IGES file CAE2IS.IGS Open the scratch file P6RTMP4 to be used for overflow should the file length exceed the NBUF $=3000$ limit. Read the spline and hope for the best. Close the IGES file and give thanks to the IGES gods.

4. PUT_IGES (IG_SPL, NSPTS, SPL_TYP)

INPUT PARAMETERS:

IG_SPL - Array containing spline data

NSPTS - Number of spline points
SPL_TYP - Type of spline being written OUTPUT PARAMETERS:

None

This subroutine puts (or writes) an IGES formatted file. IGES files contains five sections that each have a unique structure. The first section is called the GLOBAL section and provides hardware/machine dependent information.

The second section is called the "START" section and contains information about the data, such as plot titles, etc.

The third section is called the DIRECTORY ENTRY section and contains information about each of the geometry entities in the file, such as type of entity, label, how it should be displayed, level it lives on, etc. The DE section also contains a pointer to the Parameter Data (PD) section where the coordinate data for the geometry live.

The final section, called the TERMINATOR section, contains an accounting of how large each of the other sections were.

Each section is disseminated by different letters in the 72nd column of each line. The global section has a $\mathrm{G}$ in column 72 , the start section an $S$, the directory entry section a $D$, the parameter data section a $\mathrm{P}$ and the terminator section a $\mathrm{T}$.

The actual coordinate data is stored in the PARAMETER DATA section. For splines, the format of the 112 and 126 entities can vary. Even the interpretation of the 112 entity can vary depending on the spline type being read. 
This subroutine assumes that the IGES output file is called IS2CAE.IGS and contains a single spline entity of type 112. A full IGES WF spline definition is stored in the IGES file.

\section{IGES_IN (IG_SPL, NSPTS, SPL_TYP)}

INPUT PARAMITTERS:

None

OUTPUT PARAMETERS:

IG_SPL - Array containing spline data

NSPTS - Number of spline points SPL_TYP - Type of spline input

This subroutine reads an IGES file, determines the spline type used to store the spline, and loads the spline array.

A full spline (defining points and coefficients) is assumed: anything less would be of little use. Read Start and Global sections of the IGES file (File is already open). you don't care about the contents, only with how many records each contain.

The NI_ parameters are the count for the number of records for each section. The NS_parameters indicate what line in the IGES file each section begins. GET ENTITY TYPES $(100,102, E T C$.$) Read the DE section$ records and load the DE-arrays.

If you don't have an entity, you have nothing to read. Read a 112 spline Probably a Wilson-Fowler spline. Read a 126 spline - Probably a Cubic B-spline. Read a Composite Curve.

The variable $\mathrm{N}$ is the number of entity pointers in the DE record. Reading IGES files is not for the weak at heart.
6. READ_IGES (NIG, NIS, NID, NIP, LREC, NSS, NSG, NSD, NSP, ABUF, ID1BUF, ID2BUF, TYPBUF)

\section{INPUT PARAMETERS:}

None

\section{OUTPUT PARAMETERS:}

NIS -

Number of Start Section records

NIG - Number of Global Section records

NID - $\quad$ Number of Directory Entry records

NIP - Number of Parameter Data records

LREC - Record limit (last record) on IGES random file

NSS,NIS - Start record, record count for S-records

NSG,NIG - Start record, record count for G-records

NSD,NID - Start record, record count for D-records

NSP,NIP - Start record, record count for P-record

This subroutine reads an IGES file. The name of the IGES file is CAE2IS.IGS, and is hard-coded into the algorithm. The algorithm scans the IGES file and determines the length of the start and global sections.

This algorithm originated in the IDEAL library of WX-Division at the Los Alamos National Laboratory.

Initialize some of the necessary parameters. Read sequential file in the implied do-loop. 
7. READ_DE (NREC, NID, NSD, DPAR, LABEL, ABUF, IDIBUT, ID2BUF, TYPBUF)

INPUT PARAMISTERS:

LIN - Unit number for opened IGES input file

NREC - Logical record number in IGES file (D-REC number = 2*NREC-1)

\section{OUTPUT PARAMETERS:}

IDPAR(1) and IDPAR(11) IGES record type (type of entity)

$100=$ circular arc

102 = composite curve

$104=$ conic arc

106 = copious data

108 = plane entity

$110=$ line entity

112 = parametric spline

$114=$ parametric spline surface

$116=$ point

118 = ruled surface

$120=$ surface of revolution

122 = tabulated cylinder

124 = transformation matrix

126 = B-spline

IDPAR(1) - Entity type

IDPAR(2) - Parameter record pointer

IDPAR(3) - Version

IDPAR(4) - Line font pattern

IDPAR(5) - Level

IDPAR(6) - View pointer

IDPAR(7) - Defining matrix pointer

IDPAR(8) - Label display pointer

IDPAR(9) - Status

IDPAR(10) - Seq \#

IDPAR(11) - Entity type (same as IDPAR(1))

IDPAR(12) - Line weight

IDPAR(13) - Pen number

IDPAR(14) - Parameter record count

IDPAR(15) - Form number

IDPAR(16) - Reserved
IDPAR(17) - Reserved

IDPAR(18) - Entity label

(IDPAR(18)=0)

IDPAR(19) - Entity subscript

IDPAR(20) - Seq \# (IDPAR(10)+1)

This subroutine reads the Directory Entry (DE) section of an IGES formatted file. The algorithm assumes that a file has already been opened in unit=LIN. There are 20 parameters in a DE record. The the fields of a DE record are integers except field 18 which is a character. When the DE record is loaded into the IDPAR array the 18th field is set to zero.

This algorithm originated in the IDEAL library of WX-Division at the Los Alamos National Laboratory. The subroutine was modified by Ron Dolin beginning on 7/8/92.

Parameters ID1 and ID2 are integers, NSD is the line number in the IGES file where the DE section begins.

8. PGRRAF (IREC, A, ID1, TYPE, ID2, ABUF, ID1BUF, ID2BUF, TYPBUF)

\section{INPUT PARAMETERS:}

IREC - IGES physical record to read

\section{OUTPUT PARAMETERS:}

A - 64-by array from IGES record

ID1 - 8-by back pointer to IGES Drecord (valid only in P-record)

TYPE - IGES section type

ID2 - Record number within IGES section (integer)

This subroutine reads a line of input from the open IGES file and sends it back to be interpreted.

This algorithm originated in the IDEAL library of WX-Division at the 
Los Alamos National Laboratory. It was modified by Ron Dolin beginning 7/8/92.

9. READ_WF (IRREC, ND, NSD, NSP, ABUF, ID1BUF, ID2BUF, TYPBUF, IG_SPL, NGPTS)

INPUT PARAMIETERS:

IRREC - Sequential record number on IGES file

\section{OUTPUT PARAMETERS:}

NWFPTS - Number of coefficients read (T(1)...T $(\mathrm{N}+1)$, and $\mathrm{C}(1,1,1) \ldots \mathrm{C}(4,3, \mathrm{~N}+1))$ (note: $\mathbf{N}$ is 1 greater than $\mathbf{N}$ returned by P6R112)

WF_SPL - Coefficients of the WF spline (reorder from $\mathrm{T}(1) . . \mathrm{T}(\mathrm{N}+1), \mathrm{C}(1,1,1) \ldots$ $\mathrm{C}(4,3, \mathrm{~N}+1)$ order to $\mathrm{T}(1)$, $\mathrm{C}(1,1,1) \ldots \mathrm{T}(\mathrm{N}+1)$, $\mathrm{C}(1,1, \mathrm{~N}+1) \ldots$ order $)$

IER - $=0$ on good read $=1$ if IRREC not 112

This routine calls P6DR112 for D. L. Jaeger's Wilson-Fowler reader. The following set of routines were modified for double precision by R. M. Dolin.

10. READ_112 (IRREC, NID, NSD, NSP, ABUF, ID1BUF, ID2BUF, TYPBUF)

\section{INPUT PARAMETERS:}

IRREC - Logical record to read from IGES (IDREC $=2 *$ IRREC-1)

\section{OUTPUT PARAMETERS:}

CTYPE - Spline type

TYPE 1 = Linear

TYPE 2 = Quadratic

TYPE 3 = Cubic

TYPE 4 = Wilson-Fowler
TYPE 5 = Modified WF TYPE 6 = B-Spline

H - Degree of continuity with respect to arc length

NDIM - 2 = Planar, $3=$ Non-planar

N - Number of points or point sets in array $P$

T - Break points of piecewise polynomial

C - Parametric cubic coefficients at the break points

IER - $\quad=0$ Then good read $=1$ Then record requested to read is not type $\$ 106$

This subroutine reads IGES 112 spline entities. It was written by Rob Oakes for IDEAL, and was modified for the IS program by Ron Dolin on $6 / 16 / 92$.

Get requested D-record. If a valid record, read parameter data. The variable NSP defines line \# of first parameter data record. IDPAR(2) defines $P E$ record spline begins at. IDPAR(14) defines \# of PE records used by spline. IR1 is the start line of spline (in file's line count).

IR2 is the end line of spline (in file's line count). If IR2 is greater than 400 , one must split read into multiple sections (string array limits). Read file line by line. Output parameters. Stuff parameters based on entity type.

11. DFREFLD (S, C, V, N, IV, NV, IER, DV)

INPUT PARAMETERS:

S - Character string

IV - Array for flag, $0=$ real, $1=$ string 
NV - Maximum number of variables that user can read

OUTPUT PARAMETERS:

C - Array for return of string variables

V - Array for return of real variables

N - Number of variables read

IER - Error flag - 0mgood read, 1=bad line, 2=too many variables

DV - Array for return of real double precision variables

This subroutine is a free field reader originating from the IDEAL library of algorithms. This algorithm is a special version of the free field reader optimized for point defined geometry data decks.

This subroutine was originally developed for the IDEAL library by $D$. L. Jaeger of LANL, and then modified by Jaeger for the PET software. It was then further modified for the IS program by R. M. Dolin on 10/22/91.

12. IGES_OUT (IG_SPL, NSPTS) INPUT PARAMETERS:

IG_SPL - Array of the spline

NSPTS - Number of spline points OUTPUT PARAMETERS:

None

This subroutine writes an IGES file for a Wilson-Fowler spline. This means that the IGES 112 entity will be used. A full WF spline will be written, i.e., defining points and coefficients. This is a double precision version of our singles sensation.

Initialize the output file. Spline name will be SWX1234 for now. IDREC is a directory entry pointer.
Convert DP version of epline to SP version.

18. POWINI (IDF, IPF, NTITLE, TITLE, ID, IP)

INPUT PARAMETERS:

IDF - Unit number for file1

IPF - Unit number for file2

NTITLE - Number of characters in title

TITLE - Character array of title OUTPUT PARAMIETERS:

ID - Unit file1 reads to

IP - Unit file2 reads to

This subroutine initializes files for IGES output. Get the machine dependent parameters.

Get IGES file. Hard code file name SE2CAE.IGS into file name.

Output title records, count number of title records.

\section{P1LOWR(B)}

\section{INPUT PARAMETERS:}

B - Character string

\section{OUTPUT PARAMETERS:}

P1LOWER, - Modified string

The function P1LOWR(B), has entries for P1UPPR(B) and P1CASE(B).

The function converts characters to lower case. P1LOWR() has an entry to P1UPPR that converts characters to upper case. There is also an entry for P1CASE. you do not really know the difference between P1UPPR and P1CASE.

This function was written by Rob Oakes or Dwight Jaeger in the 1980's, 
and modified for the SE module by Ron Dolin beginning on 8/25/92.

\section{P1LADJ (CARG)}

\section{INPUT PARAMETERS:}

CARG - Character string unadjusted

\section{OUTPUT PARAMIETERS:}

CARG - Character string adjusted

This subroutine left adjusts the input character string by removing preceding blank spaces.

The algorithm also has entries for P1RADJ(CARG) that right adjusts the input string by truncating the string after its last nonblank character.

This subroutine was written by Rob Oakes or Dwight Jaeger in the 1980's, and modified for SE module by Ron Dolin beginning on $8 / 25 / 92$.

16. P6WWFS (NAMT, LEVEL, MATRE, NPTS, SPL, IDREC, ID, IP, IPF, IPF)

INPUT PARAMETERS:

NAME - Entity name

LEVEL - IGES level for this entity

MATRIX - Definition space

NPTS - Number of spline points

SPL - Array of spline data OUTPUT PARAMETERS:

IDREC - Directory record pointer for the entity

ID - D-record index in Dfile

IP - P-record index in Pfile

IDF - D-record file number

IPF - P-record file number
This subroutine is used to call P6W112 using D.L. Jaeger's convention for Wilson-Fowler spline formal parameters. This version of P6WWF is in double precision.

This subroutine was written by Rob Oakes or Dwight Jaeger in the 1980's, and modified for SE module by Ron Dolin beginning on 8/25/92.

Load the $T$ array. The $T$ array contains the spline's defining points. Write that spline and restuff the P-array back to the way it came in.

17. PoW112 (NAMIS, LIVEL, MATRE, CTYPE, H, NDM, N, T, C, IDREC, ID, IP, IDF, IPF)

INPUT PARAMETERS:

NAME - Entity name

LEVEL - IGES level for this entity

MATRIX - Definition space

CTYPE - Spline type

$$
\begin{aligned}
& 1=\text { Linear } \\
& 2=\text { Quadratic } \\
& 3=\text { Cubic } \\
& 4=\text { Wilson-Fowler } \\
& 5=\text { Modified Wilson-Fowler } \\
& 6=\text { B spline }
\end{aligned}
$$

NDIM $-2=$ planar

$3=$ non-planar

H - Degree of continuity

$\mathrm{N}$ - Number of segments in spline (dimensionality of CX, CY, CZ is $(4, N+1))$

T - Break points for piecewise polynomial

C - Array of spline coefficient data to be output (coefficients are in order expected by IGES file)

OUTPUT PARAMETERS: 
IDREC - Directory record pointer for the entity

ID - D-record index in Dfile

IP - P-record index in Pfile

IDF - D-record file number

IPF - P-record file number

Internal variables:

ITYPE - Ropresents D-REC encry *1 and $\# 11$, entity type number

DSEQ1 - D-Rec entry *10, sequence number

DSEQ2 - D-Rec entry *20, sequence number

PSEQ - P-Rec sequence number

LWEIGHT - D-Rec entry \#12, line weight

NPEN - D-Rec entry \#13, pen number

NPREC - D-Rec entry \#14, number of records in P-data for this entity (related to $\mathbf{N}$ for \#112 spline entity)

CTYPE - D-Rec entry \#15, (see input definitions)

This subroutine writes the cubic spline into an IGES format. It was written by Rob Oakes or Dwight Jaeger in the 1980's, and modified for SE module by Ron Dolin beginning on 8/25/92.

Define the data type to be used later Reject if number of segments is less than 1 . If there are too many points, you should never have gotten this far.

IFOR is the resolution of FORTRAN writes of the data. Specify the degree of continuity. you don't use T\&R matrices in the SE program.
Compute the number of P-records (parameter data) to put on the IGES file. Compute the number of $T$ values to put on the first P-record so that the last P-record will have 6 values.

Encode the D-sequence values. Replace blanks with 0 's in sequence specifiers. Write D-file records. Find last non-blank character, put the EOR there.

Output rest of the P-records having $T$ values. Output the P-records for the spline coefficients. Update ID and IP pointers.

18. PGWEND (FILE1, FIIF2, DFILE, PFIIE, NOS, ID, IP)

INPUT PARAMETERS:

FILE1 - Input file number

FILE2 - Output file number

DFILE - DE file number

PFILE - PD file number

NOS - Number of start records

ID - Number of DE records

IP - Number of PD records

OUTPUT PARAMETERS:

None

This subroutine completes and closes the IGES file that is being generated. It was written by Rob Oakes or Dwight Jaeger in the 1980's, and modified for SE module by Ron Dolin beginning on 8/25/92 .

The final record of an IGES file is the Terminator section. It contains an accounting of how many records from each section exist on the file. Set all the blanks equal to zeros in the Terminator section. Close the IGES file. 
Make different system calls depending on whether on VAX or CRAY.

19. IINPAT(STRNNG, OLDPAT, NEWPAT)

INPUT PARAMITTERS:

STRING - Input character string

OLDPAT - Input pattern string

OUTPUT PARAMETERS:

NEWPAT - Output pattern string

The function I1NPAT replaces an existing string with a new string. It was written by Rob Oakes or Dwight Jaeger in the 1980 's, and modified for SE module by Ron Dolin beginning on 8/25/92.

20. C1PRIN(I)

INPUT PARAMETERS:

I - Input integer

\section{OUTPUT PARAMETERS:}

C1PKIN - Character string of integer

This character function converts an integer into a character. Write the integer into a character field and then set the function equal to the character.

This function was written by Rob Oakes or Dwight Jaeger in the 1980's, and modified for SE module by Ron Dolin beginning on $8 / 25 / 92$.

\section{I1LSTN(CS)}

\section{INPUT PARAMETERS:}

CS - Input character string

\section{OUTPUT PARAMITTERS:}

\section{L1LSTN - Output integer of character string}

This function converts a character string to an integer. It was written by Rob Oakes or Dwight Jaeger in the
1980 's, and modified for SE module by Ron Dolin beginning on $8 / 25 / 92$.

\section{FGATAN(AY, AX)}

\section{INPUT PARAMIETERS:}

AY - Y coordinate of angle

AX $-X$ coordinate of angle

OUTPUT PARAMTETERS:

F5ATAN - Arc tangent of angle

This function computes the arc tangent of (AY / AX). The function takes care of infinite arc tangent problems. It also takes care of improper quadrant problems that can arise when using various compilers.

The value of the arc tangent is returned in degrees. This is a double precision version of the original IDEAL library function written by Rob Oakes or Dwight Jaeger in the 1980's. This function was modified for SE module by Ron Dolin beginning on 8/25/92.

23. I4MERG (F1, F2)

INPUT PARAMETERS:

F1 - First file to be merged

F2 - Second file to be merged

OUTPUT PARAMETERS:

None

Merge the two input files ( $\mathrm{f} 2$ into $\mathrm{f} 1$ ). This function was written by Rob Oakes or Dwight Jaeger in the 1980's, and modified for SE module by Ron Dolin beginning on $8 / 26 / 92$. 


\section{CHAPTER 5 SOFTWARE VERIFICATION AND VALIDATION PLAN}

The Software Verification and Validation Plan (SVVP) defines how the Is coftware is to be tented. The SVVP identifies the objectives for each teat and the conditions under which they will be run. There will be five test performed to verify both the IS theory and the quality of the IS software.

\section{A. Test 1}

The WX-Division IGES editing and mass properties program, IGVIEW, will participate in the first spline test series. IGVIEW contains an implementation of the Wilson-Fowler spline algorithm written by $R$. M. Dolin. ${ }^{1}$ IGVIEW is a single precision program. This means that evaluation results can only be compared to typically six significant digits of accuracy.

The verification and validation tests will use the Wilson-Fowler algorithm with default end conditions. Three commercial computer-aided engineering programs will also be used for the verification and validation tests. They are the ICEM, ANVIL, and Applicon CAE software systems.

The Applicon CAE system is also known as BRAVO. It contains Uniform B-spline and Nonuniform B-Spline algorithms. The verification and validation tests will use the Wilson-Fowler algorithm with default end conditions.

Three analytical topologies will be used for the verification and validation tests. The analytical topologies are a $2 x$ ellipse, 20x ellipse, and a sine wave.
Several defining point sets will be used to test each analytical clurve. The defining point sets will start out coarse (ten points), and increase in point density. The numbers of defining points used to approximate the analytical curves will be $10,20,30,45$, 90,180 , and 360 . In all cases, the defining point sets will be evenly distributed.

The tests should demonstrate that all four spline algorithms can render spline topologies with errors below the specified threshold. They will probably all display different characteristics, like converging at different rates.

\section{B. Test 2}

The second test suit will compare the APT WF program with the Dolin WF spline. In this analysis the APT WF program will be used as the base line definition. The APT WF program will not be altered during the process of taking it from the APT family of algorithms and integrating it into the IS software. This test will compare the two WF programs for every contour on the CONSYS data base.

The procedure will be to read the contour data from CONSYS into the IS program. The APT WF program will be used to generate the base line spline

1 R. M., Dolin, "The Wilson-Fowler Spline In A Global IGES Coordinate Frame," Los Alamos National Laboratory report number LA-1 1024MS, (September, 1987). 
definition and compute the end angles. The through point information from the contour and the end angles computed by the APT WF program will be passed into the Dolin WF program. This means that the base line spline will have default end angles while the Dolin WF spline will have forced end angles.

Error between the two generated splines will be the measure of the linear distance that the Dolin WF spline is from the base line spline at approximately 1500 parametric points. By definition the error will always be zero at the through points of the base line spline but can vary in the intervals between through points.

Information generated from this analysis should include the number of contours that could be made equivalent, the reasons why contours that could not be made equivalent failed, and the number of spline subdivisions required to achieve equivalence for each design contour.

\section{TEST 3}

The third test to be performed will verify that the IS technology preserves the integrity of the Division's historical designs. This is done by showing that an APT WF spline can be made equivalent to ICEM, BRAVO, and IGVIEW defined splines. For these tests the APT WF spline will be the base line definition and the ICEM, BRAVO, and IGVIEW generated splines will be made equivalent to the base line. The sample size for this test suit will be sixty-six historical design contours with end angles defined by design drawings.

Information generated from this analysis will include the number of contours that could be made equivalent, the reasons why contours that could not be made equivalent failed, and the number of spline subdivisions required to achieve equivalence for each design contour.

These tests should verify that the APT WF generated splines and BRAVO generated B-splines can be made equivalent. The tests will also demonstrate that APT WF splines and ICEM-generated splines can be made equivalent. Finally, the tests will show that BRAVO-generated B-splines can be made equivalent to ICEM-generated splines using the IS software as an "honest broker."

The analysis will demonstrate that nonWF splines can be made equivalent to WF splines. The analysis will also demonstrate the spline procedures that the Division will use in its new CAE environment. This includes the use of BRAVO and WFSYS, and how they will interact with the IS software. It also includes the use of ICEM and how the design to manufacturing process works.

The analysis should help define the process used to generate new spline topology, preserve historical designs, and migrate from the way we "used to do business" into a new environment. 


\section{CHAPTER 6 SOFTWARE VERIFICATION AND VALIDATION REPORT}

The software verification and validation report (SVVR) presents the results of the tests outlined in the software verification and validation report (SVVP). The SVVP identifies the objectives for each test and the conditions under which they were run. There were three series of tests performed to verify both the IS theory and the quality of the IS software.

\section{A. TEST 1}

The WX-Division IGES editing and mass property program, IGVIEW, participated in this first spline tests. IGVIEW contains an implementation of the Wilson-Fowler spline algorithm written by R. Dolin. IGVIEW is a single precision program. This means that evaluation results can only be compared to typically six significant digits of accuracy.

The verification and validation tests used the Wilson-Fowler algorithm with default end conditions. Three commercial computer-aided engineering programs were also used for the verification and validation tests. They were the ICEM, ANVIL, and Applicon CAE software systems.

The Applicon CAE system is also known as BRAVO. It contains Uniform B-spline and NonUniform B-spline algorithms. The verification and validation tests used the NonUniform B-spline algorithm.

The ICEM CAE software system has a Wilson-Fowler spline algorithm. The verification and validation tests used all default conditions with circular end angles. The ANVIL CAE software system has a Wilson-Fowler spline and a piecewise cubic spline algorithm. The verification and validation tests used the Wilson-Fowler algorithm with default end conditions.

Three analytical curves were used for the verification and validation tests. The analytical curves were are a $2 \mathrm{x}$ ellipse, a 20x ellipse, and a sine wave. The curves are shown in figure 1.

The sine wave extends from $0<\theta<$ $2 \pi$ radians and the other three curves extend from $0<\theta<\pi / 4$ radians. Figure 6.1 shows the three analytical curves used for this test series.

Several defining point sets were used to test each analytical curve. The defining point sets started out coarse (ten points) and increased in density.

The numbers of defining points used to approximate the analytical curves were $10,20,30,45,90,180$ and 360 . In all cases, the defining points were evenly distributed. The tests show that all four spline algorithms render spline topologies with errors below the specified threshold. They all display different characteristics, like converging at different rates.

The best spline approximation to the circle appears to occur when the

1 R. M., Dolin, "The Wilson-Fowler Spline In A Global IGES Coordinate Frame," Los Alamos National Laboratory report number LA-1 1024MS, (September, 1987). 


$$
\begin{aligned}
& 2 x \text { Ellipse } \\
& x=2 * \operatorname{Cos}(\text { theta }) \\
& y=\operatorname{Sin}(\text { theta) } \\
& 20 x \text { Ellipse } \\
& x=20^{*} \operatorname{Cos}(\text { theta) } \\
& y=\operatorname{Sin}(\text { theta }) \\
& \text { Sine Wave } \\
& x=\text { theta } \\
& y=\operatorname{Sin}(\text { theta) }
\end{aligned}
$$

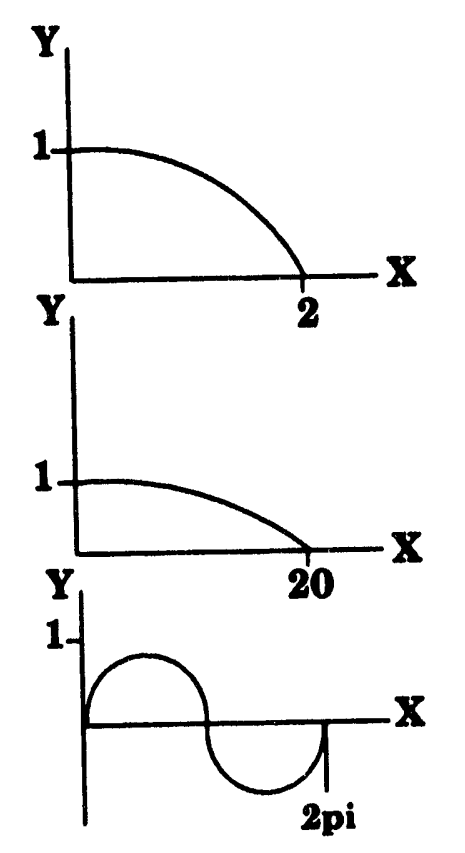

Figure 6.1. Analytical curvts used in tests.

angular increment is approximately two degrees. Decreasing the angular increment past the two degree setting does not seem to improve the spline approximation. This may suggest that there is a point of "diminishing returns" with respect to spline convergence. Figures 6.2, 6.3, and 6.4 show the spline convergence for each spline algorithm.

For the circle, the two degree angular increment is the same two degree interval typically referred to in contour specification. However, a circle is the only geometry for which this two degree phenomena was observed.
In general, the maximum error occurred at the start of the spline. Errors at spline end points is common in spline algorithms. The tests show that the Interchangeable Spline theory is valid.

\section{B. TEST 2}

The second test compared the APT WF spline program with the Dolin WF spline program. In this analysis the APT WF spline program was used as the base line definition. The APT WF spline program was not altered during the process of taking it from the APT 


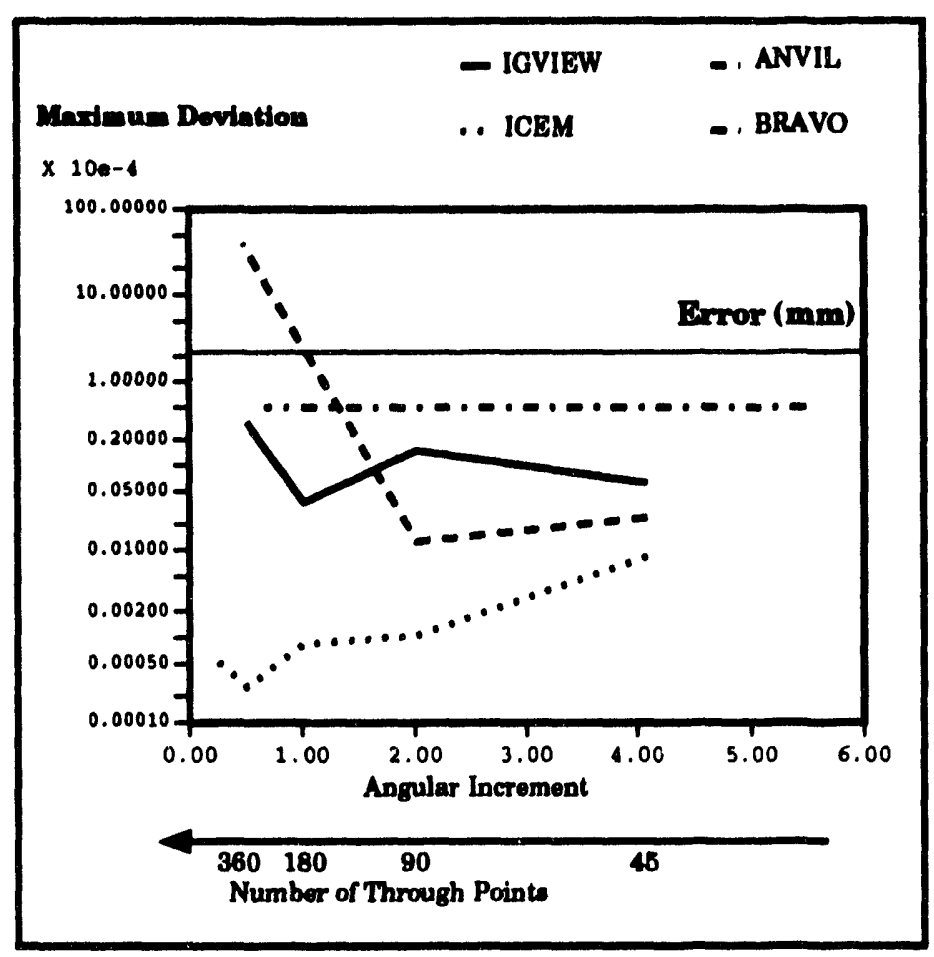

Figure 6.2. Error report for spline approximation to a $2 x$ ellipse.

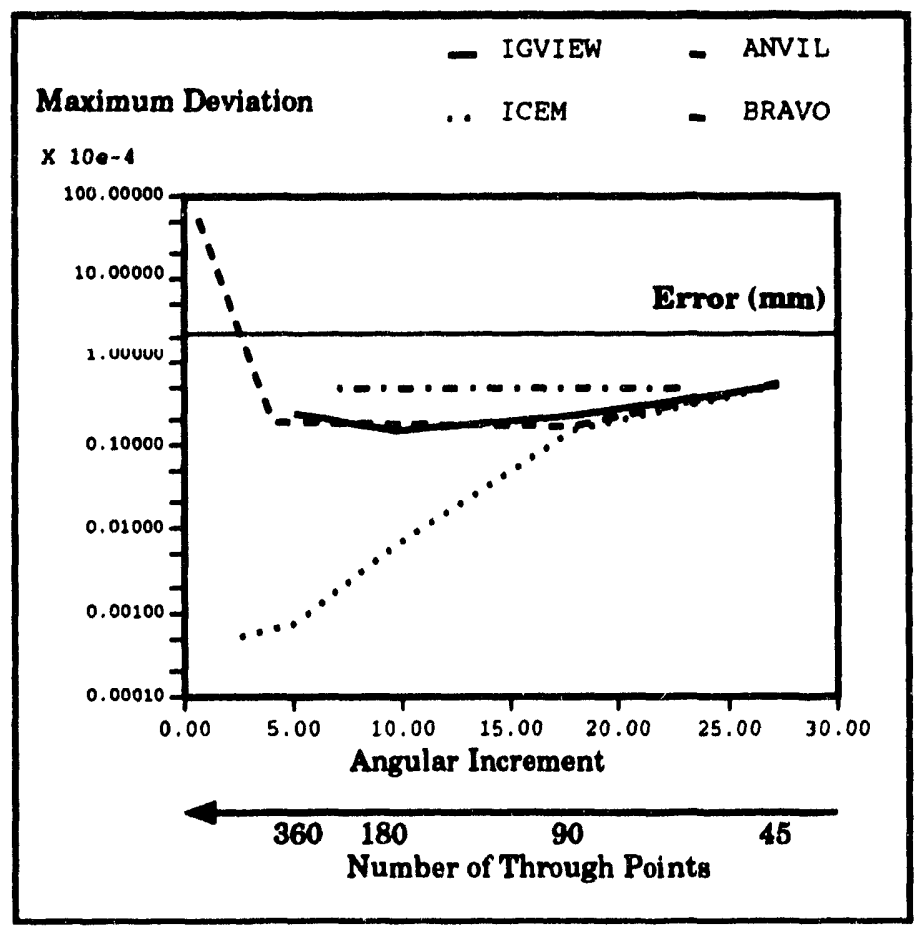

Figure 6.3. Error report for spline approximation to a 20x ellipse. 


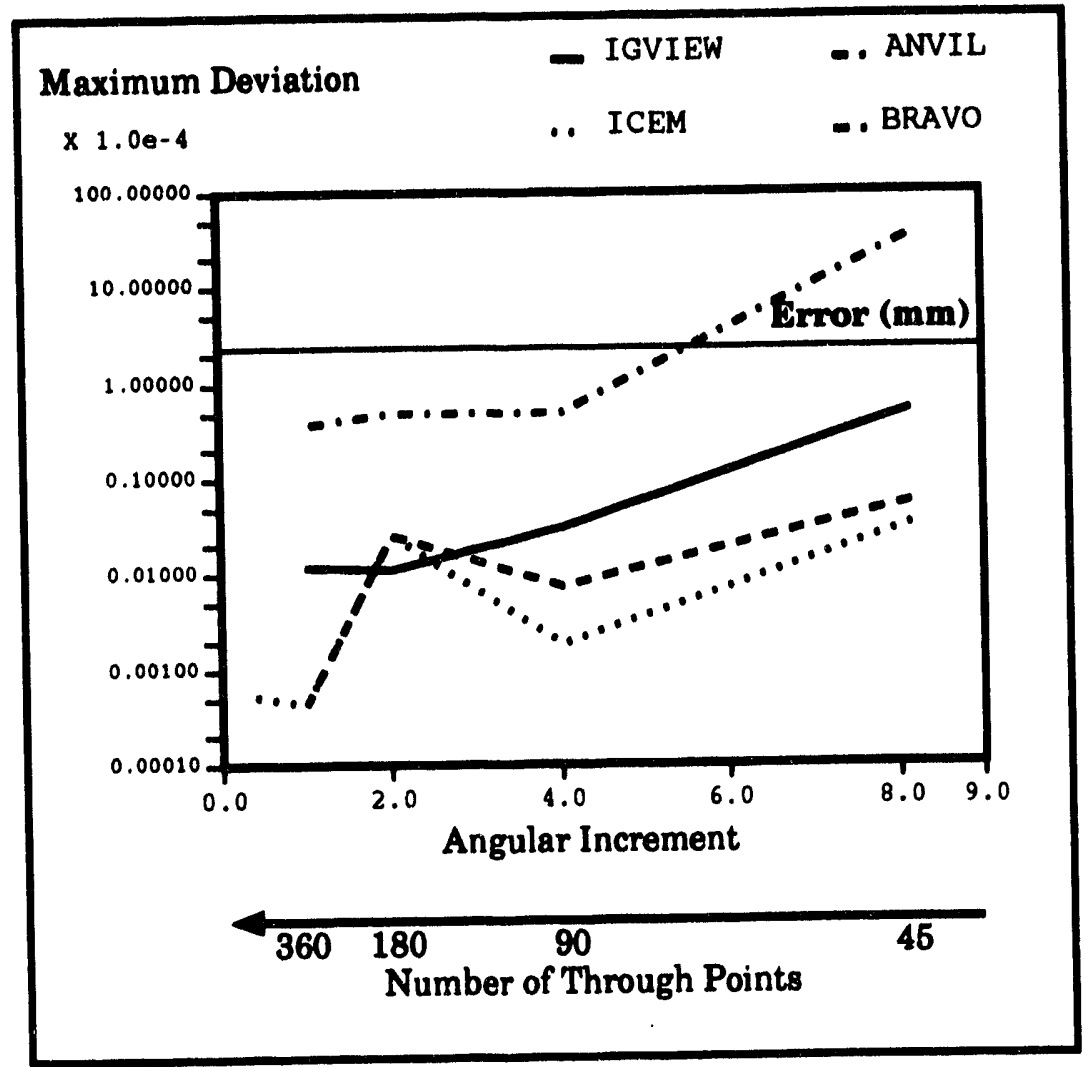

Figure 6.4. Error report for spline approximation to a sine wave.

family of algorithms and integrating it into the IS software. This test compared the two WF spline programs for every contour in the CONSYS data base.

Contour data was read from CONSYS into the IS program for each test. The APT WF spline program generated a base line spline definition for an input contour and computed its end angles. The defining point information from the contour and the end angles computed by the APT WF spline program were passed into the Dolin WF spline program. This meant that the base line spline was using default end angles while the Dolin WF spline was using forced end angles.
Errors between the two generated splines for a given contour were measured as the linear distance that the Dolin WF spline was from the base line spline at approximately 1500 parametric points. By definition the error was always zero at the defining points of the base line spline but could vary in the intervals between through points.

An attempt was made to evaluate every contour on CONSYS, however, of the 2000 eligible contours only 1554 could be attempted. There were 446 contours that were not attempted. Of those 446 contours, 126 were not 
attempted because they were of unknown type, 318 because they were empty, and 2 because there were errors in the number of points.

Of the 1554 contours that were attempted 1434 were in a Radius versus Theta (Rtheta) data format and 117 were in an XY data format. The analysis converted the Rtheta formatted files into XY formatted files. That means that 1434 contours were ultimately analyzed for equivalence. Of those 1434 contours analyzed, 1419 were processed to equivalence, and 1097 contours required additional points for the equivalent representation. In other words, 1097 IGVIEW representations required additional defining point information to generate equivalent topology.

There were 15 contours that aborted during the equivalence process. Three contours aborted because the number of data points that the contour specified containing did not match the actual number of specified points contained. Eleven contours aborted because the contour format flag was set to the XY format when the data was actually in an Rtheta format. One contour aborted because it contained coincident through points.

One of the 1434 processed contours tested, the IS software's logic for insuring that new through point positions are not too close to existing through points (closer than tolerance, i.e., $0.00025 \mathrm{~mm}$ ). The contour started out with 46 through points, ended with 81 through points, and required 101 iterations to converge. This means that the IS software attempted to subdivide the contour 101 times, but only 35 of those attempted subdivisions resulted in a new defining point.

When a spline interval is selected by the IS program for subdivision, a new defining point is located in the center of current interval, thus creating two intervals with equal segment lengths. By definition, an interval cannot have a segment length less than tolerance. If subdividing an interval results in a new interval that has a segment length less than tolerance the subdivision is not allowed.

Disallowing a subdivision creates an dilemma, the interval selected for subdivision is chosen because the spline curve through the interval is not equivalent to the base line spline's curve through that segment. So if the interval cannot be subdivided, how can equivalence be met? The IS program makes use of the following lemma to address this dilemma:

Lemma 1: When an interchangeable spline contains an interval whose curve is not equivalent to the base line spline definition's curve over the same segment length and the interchangeable spline's interval cannot be subdivided, modifying intervals in the neighborhood of the nonequivalent interval can lead to equivalence.

For the 1097 modified splines, the mean of the number of additional points was 8.7 and the standard deviation was 13.2. This means that on average, each modified spline required 8.7 additional defining points.

One concern with using the IS technology was that the number of additional points required to generate equivalent topology would be large. 
This first experiment demonstrates that the number of required additional defining points is not large.

The largest number of additional points was 99. However, when the baseline topology was investigated, discontinuities were found. When the discontinuities were removed from the baseline definition, the number of additional points decreased. In fact, when all splines containing discontinuities were removed from the initial data base of 1517 splines, the mean dropped off to 6.5 additional points and the standard deviation was 10.3.

This set of experiments matched a Wilson-Fowler spline to a Wilson-Fowler baseline. It demonstrated how different implementations of the same spline algorithm can render different topologies. The experiments also verifies that equivalent splines can be generated.

\section{TEST 3}

The third test verified that the IS technology would preserve the integrity of the Division's historical designs. The the test 2 experiment was repeated using three commercial CAE systems. These experiments considered a smaller subset of the spline data base consisting of 66 spline topologies.

The 66 topologies were selected by simply getting all design topologies used recently. They could therefore be considered somewhat random. The topologies processed using IGVIEW as the CAE module in Test 2 had a 60 percent modification rate. In other words, 60 percent of the topologies processed required additional points to generate an equivalent APT Wilson-Fowler/IGVIEW Wilson-Fowler spline.

The mean of the number of additional defining points required for the 66 spline topologies using IGVIEW as the CAE module was 5.2424, and the standard deviation was 5.6325. After processing, the mean of the errors in the equivalent IGVIEW splines versus the original splines was $1.8919 \times 10-4$ $\mathrm{mm}$, and the standard deviation was $0.4703 \times 10-4 \mathrm{~mm}$.

The same topologies processed using BRAVO as the CAE module had a 65 percent modification rate. In other words, 65 percent of the topologies processed required additional points to generate an equivalent APT Wilson-Fowler/ BRAVO B-Spline spline. The mean of the number of additional defining points required was 5.318 and the standard deviation was 5.4155 .

After processing, the mean of the errors in the equivalent BRAVO splines versus the original splines was $1.9276 \times 10-4 \mathrm{~mm}$, and the standard deviation was $0.4698 \times 10-4 \mathrm{~mm}$.

The same topologies processed using ICEM as the CAE module had a 71 percent modification rate. In other words, 71 percent of the topologies processed required additional points to generate an equivalent APT Wilson-Fowler/ ICEM spline.

The mean of the number of additional defining points required was 7.0151 and the standard deviation was 6.7766. After processing, the mean of the errors in the equivalent ICEM splines versus the original splines was 
Table 6.1. Results of CAE system tests.

\begin{tabular}{|c|c|c|c|c|c|}
\hline $\begin{array}{c}\text { CAE Module } \\
\text { Software }\end{array}$ & $\begin{array}{c}\text { Topologles } \\
\text { Modifled }\end{array}$ & $\begin{array}{c}\text { Mean Of } \\
\text { Additional } \\
\text { Polnts }\end{array}$ & $\begin{array}{c}\text { Std. Dev. Of } \\
\text { Additional } \\
\text { Polnts }\end{array}$ & $\begin{array}{c}\text { Moan Of } \\
\text { Error } \\
(\mathrm{mm})\end{array}$ & $\begin{array}{c}\text { Std. Dov. Of } \\
\text { Error } \\
(\mathrm{mm})\end{array}$ \\
\hline IGVIEW & 40 & 5.242 & 5.6325 & $1.8919 \times 10^{-4}$ & $0.4703 \times 10^{-4}$ \\
\hline BRAVO & 43 & 5.318 & 5.4155 & $1.9276 \times 10^{-4}$ & $0.4698 \times 10^{-4}$ \\
\hline ICEM & 47 & 7.015 & 6.7766 & $1.9231 \times 10^{-4}$ & $0.4851 \times 10^{-4}$ \\
\hline
\end{tabular}

$1.9231 \times 10-4 \mathrm{~mm}$, and the standard deviation was $0.4851 \times 10-4 \mathrm{~mm}$.

Table 6.1 lists the results of these numerical tests. The results seem to validate the concept of Spline

Equivalence. 


\section{CHAPTER 7 CONCLUSIONS}

The Interchangeable Spline Reference Guide fulfill two objectives. First, it describes the Interchangeable Spline software system, its data structure, and how to use it to insure apline compatibility acrose different algorithmic and software system platiorms. Second, the Interchangeable Ipline Reference Guide fulfills the software quality assurance guidelines set forth by the DOE and adopted by our Integrated Software Tools Team.

The Interchangeable Spline Reference Guide presents the Interchangeable Spline (IS) software system. This guide is an anthology of six separate reports. Each chapter represents a stand-alone report.

Chapter 1 conteins the IS software theory manual. It describes the mathematics and numerical approach used to develop the Interchangeable Spline technology.

Chapter 2 presents the Software Project Plan. This is the first of five documents required by the Department of Defense's Software Quality Assurance procedure. The Software Project Plan is meant to be a simple and short document that describes the problem, project goals, special considerations, development plan, and schedule.

It is understood that the Software Project Plan is written early in the software development effort and the goals, development plan, and schedule can change. The Software Project Plan is meant to serve as a non-literal document that attempts to put bounds on the project's scope.

Chapter 3 defines the software requirements. The Software Requirements Specification is written before software development begins.
Hence, the final form of the software system can look different than the form presented in the Software Requirements Specification.

The Software Requirements Specification defines the problem and presents a solution proposal. The numerical requirements are laid out, and a software development plan is defined.

The Software Requirements Specification describes how the software system is envisioned. It also describes how the numerical process will work, the data structure, and the Input/Output formats. A Test and Verification Plan is outlined, as well as other aspect of software quality assurance.

Chapter 4 contains the Software Design Description report. The Software Design Description contains in detail the software architecture. Each subroutine and function is described; their input and output parameters are introduced, along with an overview of the subroutine or function's objective.

The Software Design Description is written after the software has been developed and tested. It is meant to be a final report on the software system that can allow users to acquire the 
software and use the Software Design Description as a reference guide.

It helped knowing in advance that we would need to write this document. We structured our software so that we could just extract the comment lines and dump them into a document. Knowing that we were going to do that encouraged us to write welldocumented software.

Chapter 5 specifies the software verification and validation report. This Software Verification and Validation Plan describes in detail what tests are necessary to validate the software's numerical accuracy and validate its ability to solve its intended problems.

Chapter 6 is the Department of Energy's Software Verification and Validation Report. This report presents the results of the numerical tests defined in the Software Verification and Validation Plan.

If the test results are satisfactory, the software quality assurance process is complete. If the test results are unsatisfactory, the software must be modified to correct errors or deficiencies. Then the Software Design Description and the Software Verification and Validation Plan must be updated with the results presented in a new Software Verification and Validation Report.

This project took two years to complete. It represents the work of several different people from many different groups.

The Department of Energy's Software Quality Assurance plan is a good one to follow for software development efforts. While the SQA plan defines an ordered approach, we found that for our project, reports were developed out of sequence.

We wrote the Software Project Plan first, then the software. After the software was written and tested, we wrote the Software Requirements Specification. This report helped us to verify why did the things we did. It worid have been useful for solidifying our ideas, but this project's goals were well understood by the team members in advance. For most projects, writing the Software Requirements Specification before beginning software development would be appropriate.

We wrote the Verification and Validation Plan next, followed by the Verification and Validation Report. The final SAQ document that we wrote was Software Design Description. This is out of order but probably makes sense for most software development projects.

A last word on Software Quality Assurance: necessary but slow and laborious. We jokingly tell people that the software took us two weeks to develop and two years to QA. While this is not exactly true, it is surprisingly close to the truth. 

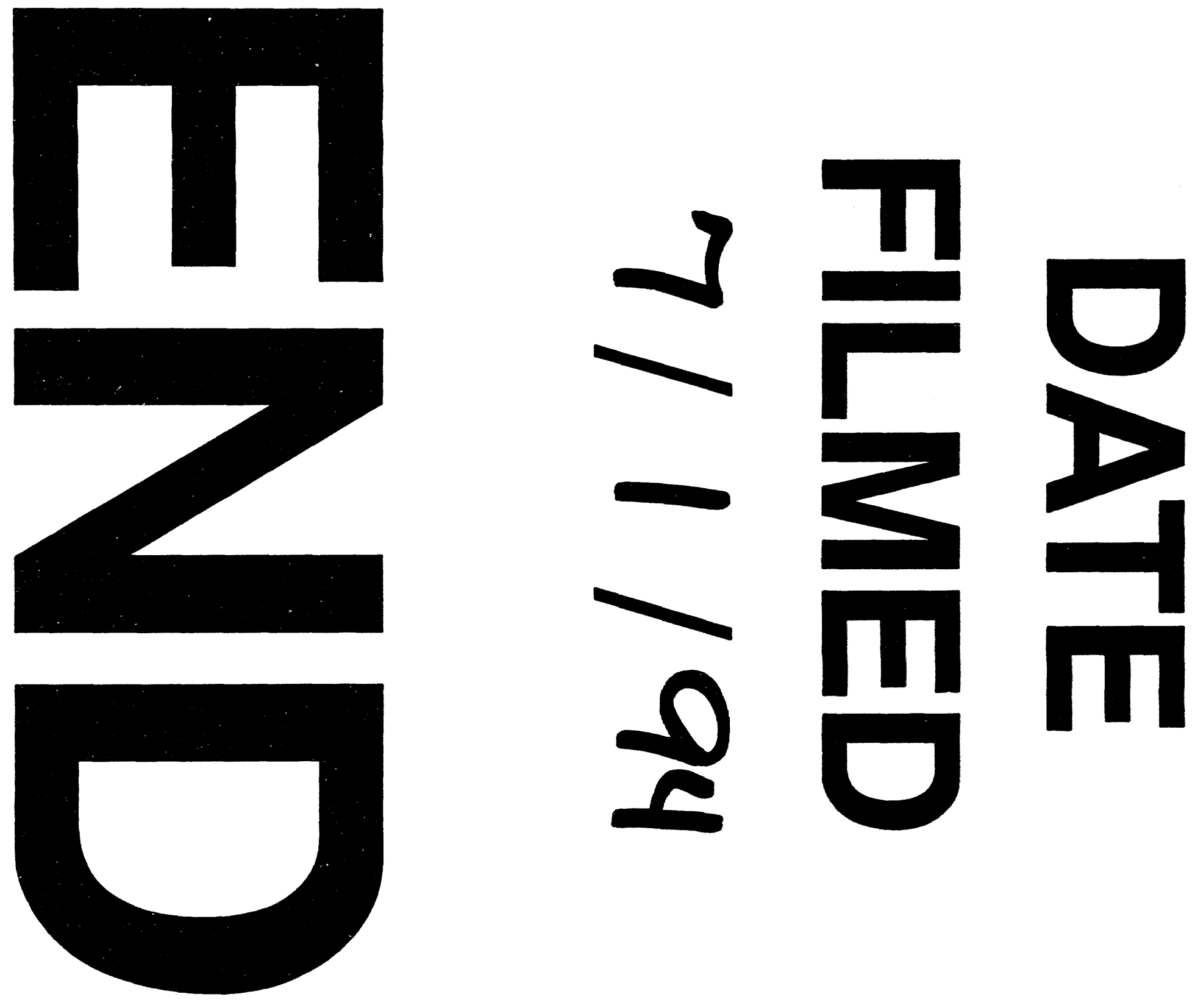
